Florida International University FIU Digital Commons

$10-31-2016$

\title{
An Investigation of the Link Between Nonverbal Aptitude and Academic Achievement
}

john e. althoff

Florida International University, john.althoff@dslca.org

DOI: $10.25148 /$ etd.FIDC001180

Follow this and additional works at: https://digitalcommons.fiu.edu/etd

Part of the Curriculum and Instruction Commons

\section{Recommended Citation}

althoff, john e., "An Investigation of the Link Between Nonverbal Aptitude and Academic Achievement" (2016). FIU Electronic Theses and Dissertations. 3054.

https://digitalcommons.fiu.edu/etd/3054

This work is brought to you for free and open access by the University Graduate School at FIU Digital Commons. It has been accepted for inclusion in FIU Electronic Theses and Dissertations by an authorized administrator of FIU Digital Commons. For more information, please contact dcc@fiu.edu. 


\section{FLORIDA INTERNATIONAL UNIVERSITY}

Miami, Florida

\section{AN INVESTIGATION OF THE LINK BETWEEN NONVERBAL APTITUDE AND ACADEMIC ACHIEVEMENT}

A dissertation submitted in partial fulfillment of the requirements for the degree of DOCTOR OF EDUCATION

in CURRICULUM AND INSTRUCTION

by

John Althoff

2016 
To: Dean Michael Heithaus

College of Arts, Sciences and Education

This dissertation, written by John Althoff, and entitled An Investigation of the Link Between Nonverbal Aptitude and Academic Achievement, having been approved in respect to style and intellectual content, is referred to you for judgment.

We have read this dissertation and recommend that it be approved.

Isadore Newman

Kyle Perkins

Eric Dwyer

$\overline{\text { Thomas G. Reio Jr., Major Professor }}$

Date of Defense: October 31, 2016

The dissertation of John Althoff is approved.

Dean Michael R. Heithaus

College of Arts, Sciences and Education

Andrés G. Gil

Vice President for Research and Economic Development and Dean of the University Graduate School

Florida International University, 2016 
(C) Copyright 2016 by John Althoff

All rights reserved. 


\section{ACKNOWLEDGMENTS}

The blessings that I have enjoyed in this life and that have allowed me to progress in my education as well as professionally are provided by God above, and I thank Him every day for all that He has provided. Additionally, I have to thank my wife for her commitment to me in this process. If it were not for her support and countless evening and weekends in which she cared for our children, I would not have gotten through this. I would also like to thank my employer, Divine Savior Academy, for supporting me in this endeavor both with time and resources.

The process of completing a dissertation is filled with numerous hours of research, writing, proofreading, and more writing. Without the support of my dissertation committee and the Office of Graduate Studies in Education at Florida International University, I would not have been able to complete this dissertation. First, I need to thank Dr. Thomas Reio, Jr. for the time he committed in working with me to proofread, edit, and finalize this dissertation, but also for his guidance in getting through the entire doctoral program and dissertation process as my Major Professor on my committee. I also would like to thank Dr. Isadore Newman for the time he committed to working with me through the methodology and statistics included in this research as well as for being part of my committee. Dr. Linda Bliss must also be commended for her contribution to this dissertation as she reviewed the manuscript and made numerous suggestions to improve its readability as well as to ensure that all questions raised were adequately answered. Dr. Eric Dwyer and Dr. Kyle Perkins were also invaluable in their suggestions for improvement throughout this process as members of my committee. I would like to 
thank a former student of mine, Maham Zafar, for the time she has spent proofreading the early versions of this manuscript. 


\section{ABSTRACT OF THE DISSERTATION \\ AN INVESTIGATION OF THE LINK BETWEEN NONVERBAL APTITUDE AND \\ ACADEMIC ACHIEVEMENT}

by

John Althoff

Florida International University, 2016

Miami, Florida

\section{Professor Thomas G. Reio, Jr., Major Professor}

When a private school works to serve a diverse student population and provide excellence in education, academic aptitude is important to evaluate to better predict whether a student will be provided an education that is appropriately leveled.

Comparing the previous grades of a diverse applicant pool may prove problematic because of the differing scaling and grading methods utilized in differing educational systems. A nonverbal aptitude test may be utilized to fill this purpose by not only gauging a student's aptitude, but also by minimizing bias associated with cultural or linguistic differences, as well as differences that may present themselves because of sex.

The present study included an ex post facto review of data collected over three academic years from 2013 to 2016 at a private secondary school. The nonverbal aptitude of applicants was gauged utilizing the Naglieri Nonverbal Aptitude Test (NNAT). The students' overall GPA during their second semester at the school was identified to gauge academic achievement. These two values were then compared utilizing a hierarchical regression analysis to identify the ability of nonverbal aptitude to predict academic achievement. Additionally, the mean values of nonverbal aptitude between the sexes as 
well as between native and non-native English speakers were compared utilizing a $t$-test. The sample consisted of 203 students. The study will add to the existing literature on the ability of nonverbal aptitude as measured by a nonverbal intelligence test to predict academic achievement in an academic setting.

The study found that the NNAT was statistically significant at the .005 alpha level in predicting academic achievement. The amount of variance explained by the overall model was $15.5 \%$ suggesting that other factors also explain the variance in academic achievement. Additionally, the t-tests performed in comparing the difference of means between the sexes as well as between native and non-native English speakers did not reflect any potential bias within the NNAT for these groups.

As part of an overall admissions process, the NNAT test can be utilized to better predict academic achievement while at the same time reduce bias toward minority students that is found in numerous aptitude tests commonly utilized in student placement processes. 


\section{TABLE OF CONTENTS}

CHAPTER

PAGE

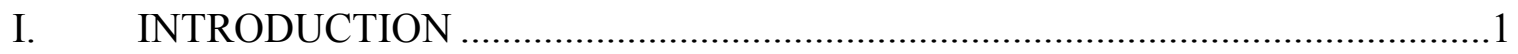

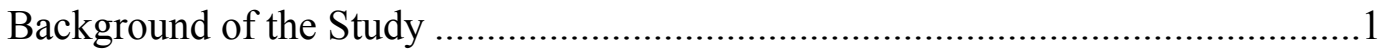

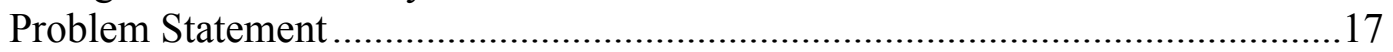

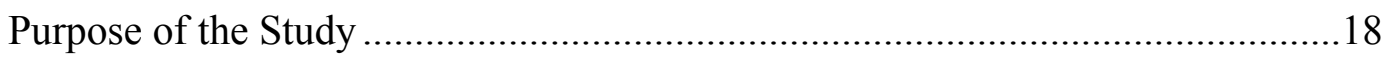

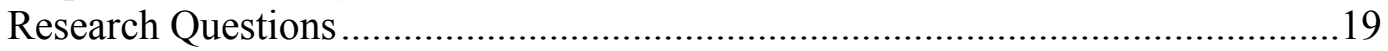

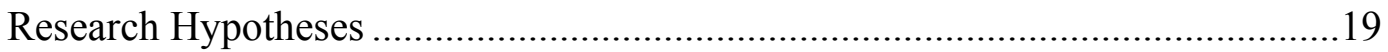

Theoretical Framework ………............................................................19

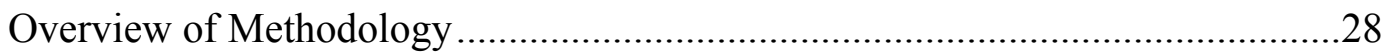

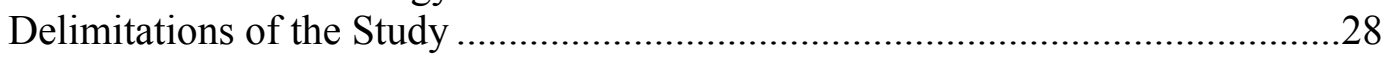

Threats to Internal and External Validity.......................................29

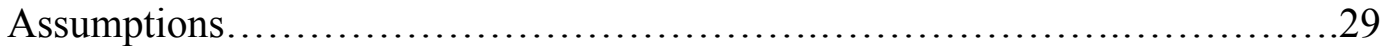

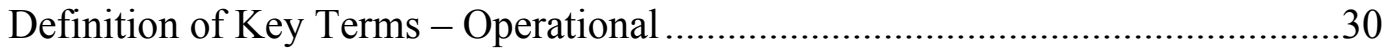

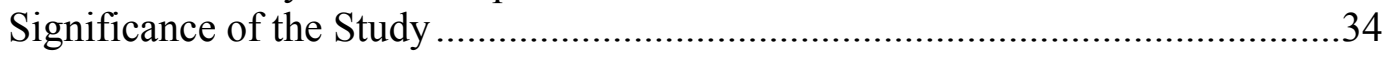

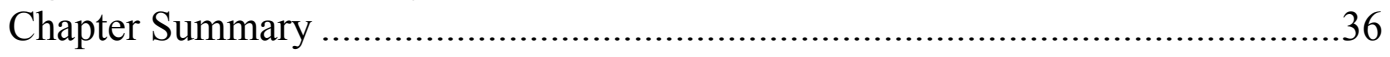

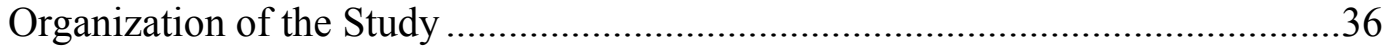

II. REVIEW AND CRITIQUE OF THE LITERATURE ...........................................38

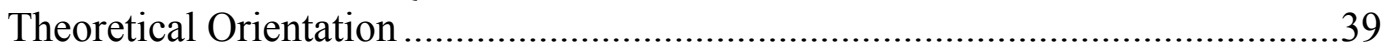

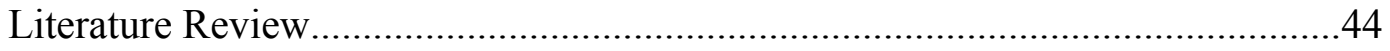

Critique of Previous Research .............................................................46

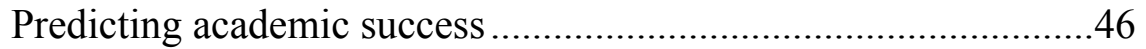

Reducing racial and economic bias..............................................50

Synthesis of Research Findings ................................................................60

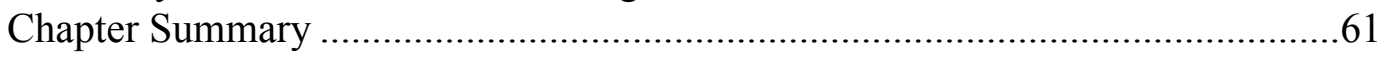

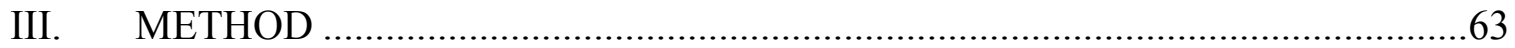

Introduction to Methodology ..........................................................................63

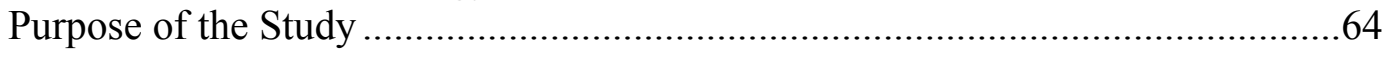

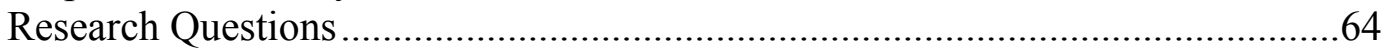

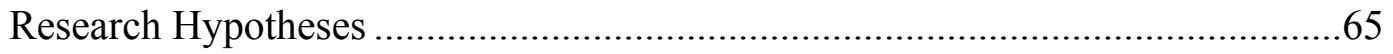

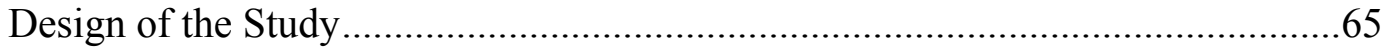

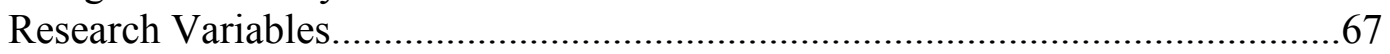

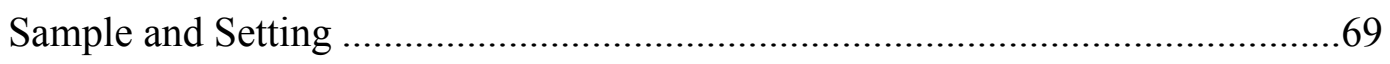

Sample Size ...................................................................................

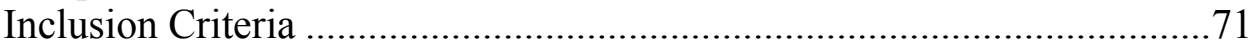

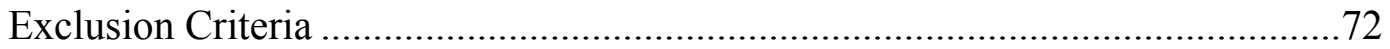

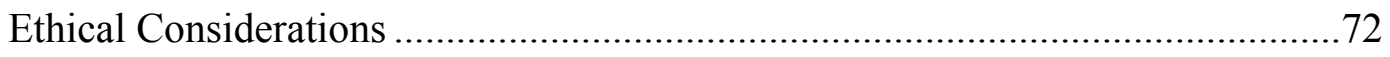

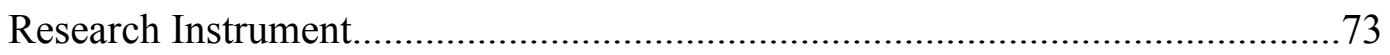

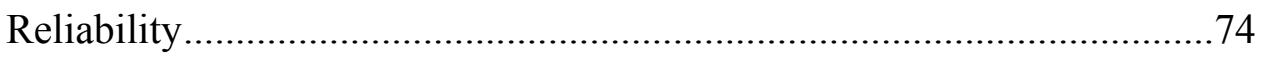

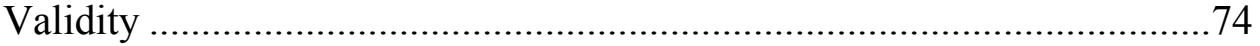




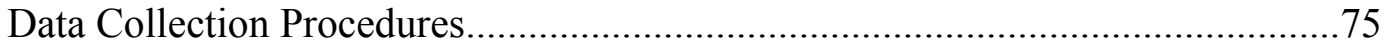

Data Analysis .......................................................................................... 76

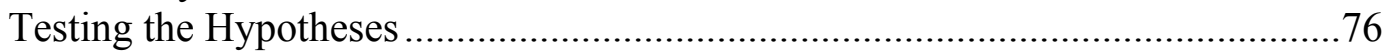

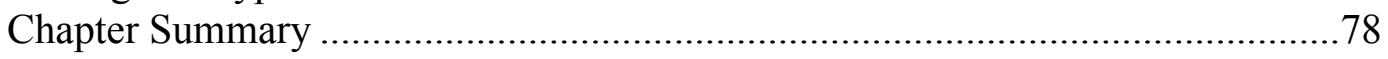

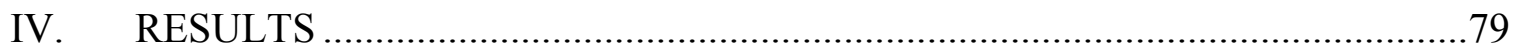

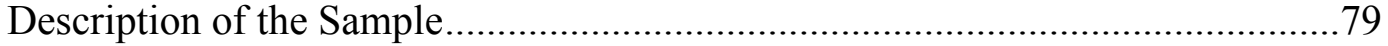

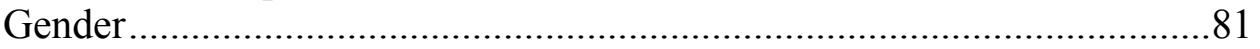

Grade Entering ........................................................................... 81

Native English Speaking............................................................. 81

$2^{\text {nd }}$ Semester GPA ................................................................... 82

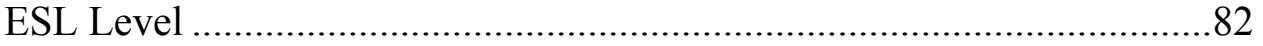

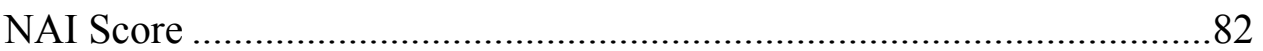

Examining the Hypotheses ....................................................................... 82

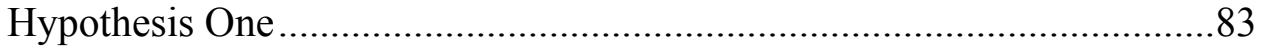

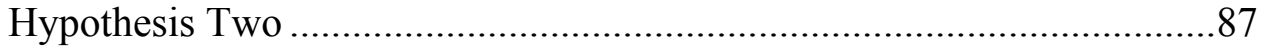

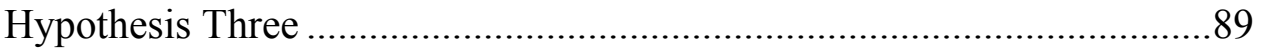

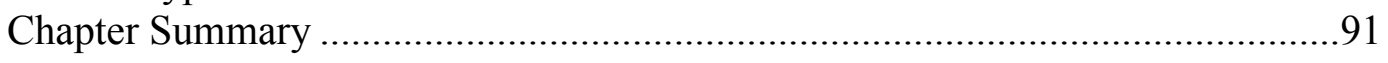

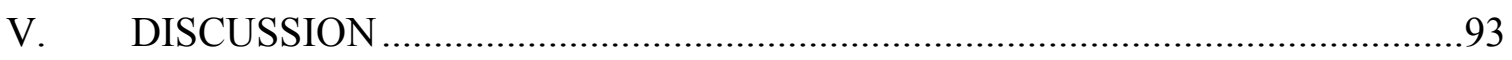

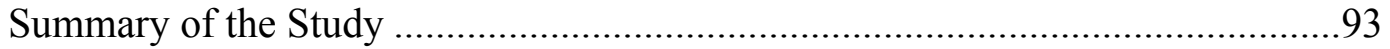

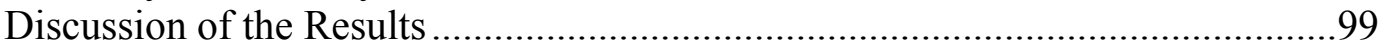

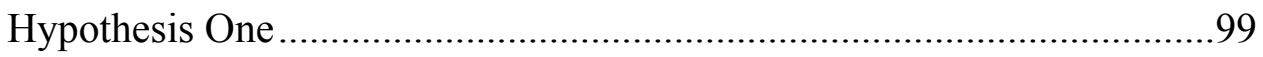

Hypothesis Two and Three .........................................................101

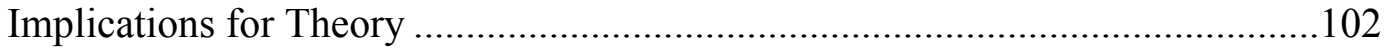

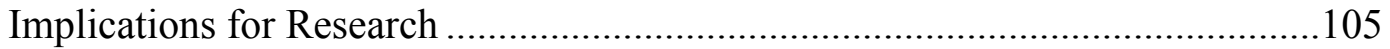

Implications for Practice ..................................................................... 107

Limitations of the Study to Generalizability.................................................109

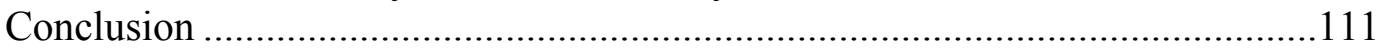

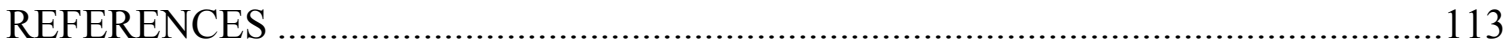

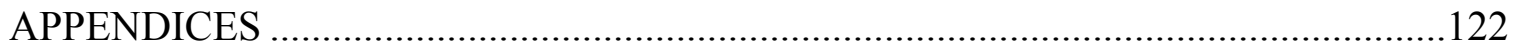

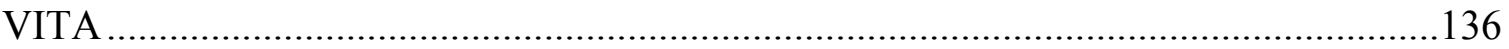




\section{LIST OF TABLES}

TABLE

PAGE

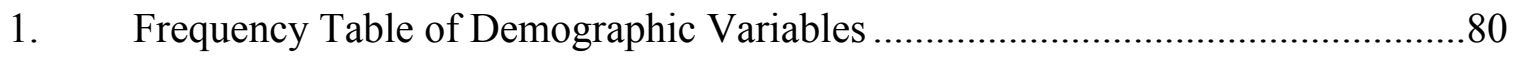

2. Descriptive Statistics of Continuous Score Variables ......................................81

3. Hierarchal Regression Coefficient Table for Semester 2 GPA ...........................84

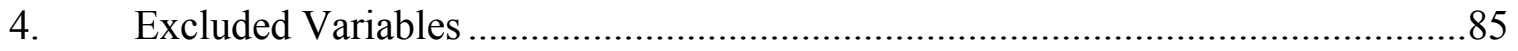

5. Intercorrelations Among Research Variables ..................................................86

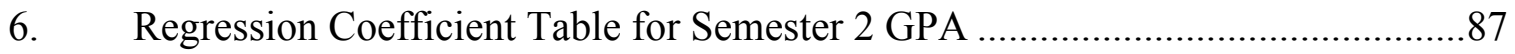

7. Group Statistics for NAI Score Based Upon Sex ...........................................8

8. Independent Samples Test for the NAI Score Based Upon Sex .........................89

9. Group Statistics for NAI Based Upon English Speaking Ability ........................90

10. Independent Samples Test for NAI Score Based Upon English

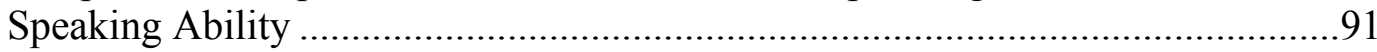

11. Full and Restricted Model Statistics for Hypothesis One ................................100 


\section{CHAPTER I}

\section{INTRODUCTION}

This chapter begins by looking at the background of the study, followed by the identification of the problem statement and the purpose of this study. The research questions and hypotheses are presented along with the theoretical framework that supported this study. A general overview of the methodology of the study is presented as well as the delimitations, assumptions that were made, and threats to validity. The significance of the study is then presented as well as the organization of the study.

\section{Background of the Study}

Private schools are established to serve a particular group of students or serve the community in a pre-specified manner. Often, this may be found in a school's mission, vision, and/or objective statements. To best fulfill these goals, the Secondary Schools Assessment Test Board (SSATB) explains that the administration of the school may highlight what a student demographic profile should reflect. A private school may purposefully work to educate students with specific educational needs, low-income circumstances, or certain religious backgrounds. Students from varied backgrounds may increasingly possess the characteristics that a private school targets.

Students applying to private schools may represent diverse backgrounds for a number of reasons. The reasons for applying may reflect diversity that already exists within the United States and the community in which a private school is located; but is also seen by the increase in international students coming to the United States to study (Open Doors, 2015). These students represent numerous cultures, subcultures, customs, and languages. These differences may highlight, promote, and strengthen differing 
intelligences within students (Verney, Granholm, Marshall, Malcarne, \& Saccuzzo, 2005). Additionally, a language other than English may be spoken as the students' primary language. These differences may pose a challenge to educators seeking to quantify the students' academic aptitude making it difficult to properly place them into appropriate academic programs that will allow students to be properly challenged and grow.

Consequently, it is increasingly important that admission directors within a private school be familiar with the population they serve. The student population includes both enrolled students as well as prospective students. Familiarity with the student population will help to ensure their practices are non-discriminatory in predicting student ability to achieve academically within a school. According to the SSATB (n.d.), admissions directors need a knowledge of the general population of the community, the private school market, area competition to include both public and private schools, as well as the school itself and its mission to best serve the school's stakeholders.

Standardized cognitive ability tests may be used to serve students in numerous ways. In the admissions area, the impact may be profound. Depending upon how they are used, however, they may have a detrimental impact on students as the results may be inappropriately used to place students in educational programs that do not meet the students' academic needs. Studies have shown that there is a disproportionately high number of minority students in special education programs (Artiles, Kozelski, Trent, Osher, \& Ortiz, 2010; Ford, 2012; Harvey \& Klinger, 2014;; MacMillan, Gresham, \& Sipersein, 1993) as well as a disproportionately low number placed into gifted programs (Ford-Harris, 1993). These disproportionate placements may be a result, in part, to the 
use of standardized tests for placement and the failure to understand the detrimental impact that biases within the test may hold for certain students who they are testing, such as students who are not of the majority: non-Hispanic Whites (Valencia \& Suzuki, 2001).

Verney et al. (2005) have explained that there is an ongoing debate in the educational community concerning the discrepancies found in standardized testing between the results of White Americans, Black Americans, and Hispanic Americans and why they exist. Hispanics have been routinely found, on average, to score approximately one half standard deviation below White Americans, while Black Americans routinely score one full standard deviation below White Americans on average on standardized tests (Camara \& Schmidt, 1999; Suzuki \& Gurkin, 1993; Whitworth \& Gibbons, 1986).

Two pervasive arguments have often made to explain these differences in test results that are commonly seen along racial and ethnic lines. The first argument states that different ethnic groups have a genetic disposition toward a certain cognitive ability level across intelligences, or $g$ factor (Rushton \& Jensen, 2003; Spearman, 1927), while the second argument is that cognitive tests expect similarities in culture and shared beliefs by the test taker to the test creators that may not exist (Kokhan, 2013; Naglieri \& Ford, 2003).

The $g$ factor is commonly incorporated into definitions of intelligence, but in light of the fact that current testing often shows bias in favor of those with a common culture and language with the test creators (Neisser et al., 1996; Jensen, 1980). In order to recognize differences in culture and language, many researchers focus more on the strengths and weaknesses of abilities displayed by individuals to recognize the numerous abilities that exist in regards to intelligence. Carroll (1993) has identified over 70 such 
abilities. Narrowing general intelligence down to just a few abilities has been seen as misleading by Ceci (1990).

Despite the trend in testing of moving away from the $g$ factor in explaining intelligence (Neisser et al., 1996), cognitive ability tests are often used within private schools by admissions officers to help quantify students' cognitive ability as part of the admissions process (SSATB). Admission officers use these tests to help fulfill the mission of the school, as well as to place students appropriately into classes and programs that will best benefit the students. In performing this duty, they may also consider students' past records, recommendations, and utilize a personal interview to gauge their potential fit within the school. Current processes, however, have led to a vast discrepancy in enrollment for minority students in gifted programs where they are underrepresented and in remedial programs where they are overrepresented (Artiles et al., 2010; Ford, 2012; Harvey \& Klinger, 2014; MacMillan et al., 1993; Naglieri \& Ford, 2003;).

When a test is administered to students with approximately equal ability, it is expected that students will receive relatively similar results. When questions on the test, or the test as a whole, present different outcomes depending on group membership of the students taking the test, differential item and test functioning is being observed (Stoneberg, 2004). Differential item and test functioning may occur because the test creators may expect certain results based upon their own background knowledge, not anticipating the background of diverse test takers. Due to this fact, an administered test may be testing completely different ideas for different groups (Huggins, 2014). When a test favors one group over another, be it because of difference in gender, race, culture, 
ethnicity, or primary language spoken, a test bias exists for the groups that receive lower or negative outcomes.

An example of testing presenting bias against minority groups due to expectations of similarities in culture by the test taker to the test maker may be seen when a student from a Latin American country takes a standardized test in the United States. There is a high probability that the student will be unfamiliar with the mathematical notation utilized to present a problem, as many common operations are taught with a very different notation system in their home countries, potentially leading to different work process despite similar results (Lopez, n.d.). Bias may exist then when unfamiliar mathematical notation is utilized extensively on the test. A few examples may better help the reader understand. The exponential form of writing one billion in the United States is $10^{\wedge} 9$ whereas in numerous Latin American countries, a billion is written as $10^{\wedge} 12$. Americans group large numbers by splitting them into groups of three separated by a comma. In Latin America, they are separated by a period or a space. Numbers following a comma identify decimal numbers rather than a decimal point. Negative numbers are written in a way that reflects a repeating decimal in the United States (Lopez, n.d.). A test utilizing such different notation would not be judging cognitive ability, but content and syntax that happened to be covered in the student's previous education. The student may be able to learn the different notation system and put it to use readily.

Another form of bias is seen as linguistic bias. Linguistic bias is specifically directed towards the speaker of a differing language from either the test administered or by the examiner. This may be associated with a student's inability to understand the test provided or the directions given by the examiner (Understanding Assessment, 2013). 
As the world continues to become smaller as a result of global trade and electronic forms of communication, people are becoming increasingly mobile, impacting education in numerous ways (Oblinger, 2001). The number of international students studying in the United States has increased in recent years. The year 2015 saw the greatest growth in 35 years with nearly 100,000 international students studying in the United States, a trend expected to continue (Open Doors, 2015). International students who want to study in the United States may not attend a public school for more than one year and must reimburse the school for the cost of education provided, and even then can only attend a school that participates in the Student and Exchange Visitor Program. Because private schools are privately funded, this limitation does not exist, as it does not place a burden on the tax-payers who contribute toward public education. Because of this, international students more commonly enroll in private schools (Study in the States, n.d.).

Davies (2011) has stated that the native speaker's concept of language has an important impact on testing. The person being tested must comprehend the language being used on the test, and this is through an understanding of the community's use of language (Davies, 2011). If the meaning behind a test problem is in the community's voice, or the test creator's, and a student is unable to interpret the meaning of the question, then that particular item is not testing what it was intended to test (Understanding Assessment, 2013). For this reason, testing aptitude should be done utilizing the student's native language or in a manner that would reduce potential language bias. 
One way a school may seek to minimize test bias is to utilize prior academic achievement as identified by previous grades to accept and place students into varying programs. In a diverse school that serves students with differing backgrounds and from numerous educational systems around the world, comparing previous grades proves problematic for a number of reasons. The first issue may be the need to translate a student's report card into English. Once that is done, differences in educational systems present themselves. In the United States, credits are used to track graduation, and classes generally are offered by the semester along with corresponding credit. In other countries, classes may be split over multiple years and/or only meet on a limited basis (see Appendix A). The students' courses may not be specific to a particular focal area within a subject. One example of this would be the common practice of teaching mathematics in many South American countries. Each year of high school reflects a mathematics grade on the report card, but this does not reflect content within the field. In these countries, students also see different aspects of Algebra, Geometry, Trigonometry, etc. to a varying degree. In the United States, students are exposed to two years of Algebra, one year of Geometry, etc. In science, it is common in South America to take a day or two of each week for Chemistry, Physics, and other topical areas, as opposed to one year of Chemistry and one year of Physics. A third difference may be the raw scores. In the United States, a 4.0 scale is most commonly used. Elsewhere, scales of 1-10 and 1-20 are utilized. The scores cannot be scaled because the numbers are used to convey different ideas (see Appendix B). Because of language barriers, differences in expectations, scheduling variations, and differing scales for grading, utilizing report cards alone make 
it difficult to utilize students' past academic achievement in order to predict future academic achievement.

The understanding of test bias and its impact on gauging intelligence that an administration or admissions officer holds may guide them in selecting what they deem to be appropriate cognitive ability tests. As a result, the tests that are utilized may produce disproportionate results along racial and ethnic lines if the tests chosen are not selected without taking the reduction of bias into consideration. Administrators and admissions officers who recognize the possible cultural and linguistic biases that tests may hold and want to promote diversity within their schools and programs may look to identify cognitive ability tests they believe reduce bias while maintaining the ability to predict academic success (Verney et al., 2005).

In addition to working to reduce bias in testing because of differences in culture race, and/or ethnicity, one must be cognizant of any bias that may exist on the testing results because of gender. Testing that is also able to reduce biases determined by gender may be a better fit in an admissions process. One must be cognizant of the fact, however, that differences alone in scores may not identify bias. The results may also be related to variables not considered in this study, such as work ethic or motivation; therefore, any differences that are seen should not be ignored (Meade \& Fetzer, 2009).

Recent studies in England have shown that when working with a given sample of elementary students, a gender gap exists in their national reading tests. The girls scored substantially higher than boys of the same age at the same grade level in literacy-based subjects. In the mathematical and scientific subjects, the results between boys and girls 
were negligible. These trends were observed over the course of the last 60 years in England (Department for Education and Skills, 2007).

The same gender difference has presented itself in international testing as well. The Programme for International Student Assessment (PISA) and the Progress in International Reading Literacy Study (PIRLS) regularly conduct research analyzing educational assessment in numerous countries. These studies have shown elementary grade female students outperforming their male counterparts in numerous recent tests (Kirsch et al., 2002; Mullis, Martin, Gonzalez, \& Kennedy, 2003; Mullis, Martin, Kennedy, \& Foy, 2007).

A gender gap is also identified when one looks at the ability of standardized tests to predict achievement at the college level. Guion (1998) documents a systematic difference in performance between genders when analyzing test scores for college admissions. Women typically obtain a higher college grade point average (GPA) than men with the same score. This discrepancy is known as predictive bias and can also be the result of test bias or because of a relationship with variables not included in this study (Meade \& Fetzer, 2009).

Elert (1992) tells us that no one would try to describe a student and his or her potential in a single sentence. It seems that tests are commonly used to do this very thing in a numerical format. This in and of itself can also promote inequality amongst students, especially in those with differing backgrounds. For this reason, it is important that admissions officers evaluate potential students based upon numerous characteristics and not solely limit their decision-making process to a single variable or test. Studies have shown us in the past that motivation amongst other factors can be an important factor 
attributed with academic success (Elert, 1992). Therefore, academic ability or aptitude testing is important and should be included in the evaluation of a student, but should only be one part of the overall evaluation (Carman \& Taylor, 2010). At the same time, aptitude testing should reduce known biases to best support the students.

To predict the ability of a student to achieve academic success by use of a test can be a challenge within a homogenous population. The challenge seen may be inferred by correlations of .23 through .37 of the SAT with the freshman GPA (fGPA) earned in college when unique demographic groups are analyzed (McDonald, Newton, Whetton, \& Benefield,, 2001). Because of the number of factors involved in predicting academic success, a .23 to .37 correlation between the SAT score and fGPA is substantial (Cohen, 1988). In a school that works to serve a diverse student population and provide a rigorous program, the task can be even more challenging. Academic aptitude is one of numerous predictors that are important to evaluate to predict whether a student will be provided an education that is appropriately leveled.

Achievement, regardless of how it is measured, is impacted by numerous variables. Motivation, grit, and self-discipline may all have an impact (Duckworth, 2006). It may also include both personal and social factors, as well as their interaction (Bandura, 1989). As a result of the number of variables that may influence one's achievement, isolating these variables and identifying their predictive ability can then be valuable as they may give a better view of the overall student when each variable is better understood in its ability to predict achievement. In isolation, their predictive power, according to Cohen (1988), may be small. Holistically, however, admissions may be 
better equipped to make decisions in regards to a prospective student when each variable has been analyzed.

Numerous tests are available to quantify a student's academic ability and may be used during the admissions process. The words intelligence, aptitude, and ability are all too often used interchangeably in testing (Macklem, 1990). The discrepancy found in the application of these words present a problem, as it can be hard to pinpoint the exact meaning of these words in their usage as well as identify the intended purpose of a particular test or battery of tests when they are used in this manner. The tests that utilize these words range from those that gauge a student's Intelligence Quotient (IQ) or intelligence across a broad range of skills and subjects, their multiple intelligences, to those that assess a student's potential or aptitude. An additional differentiating characteristic is the degree to which they use language or other cultural cues within the test itself. Some depend heavily on language and others seek to completely avoid it. While some of these tests are used to test previous academic performance and others seek to predict potential ability, both are often used to predict future academic achievement. Two well known tests are the "IQ" tests and the Scholastic Assessment Test (SAT). In private schools, however, the Secondary School Assessment Test (SSAT) is a common tool used to predict future academic performance that was created for these institutions. However, taking into consideration the changing demographics of applicants to private schools, other tests may need to be considered.

Intelligence Quotient tests are often used to predict academic achievement. In fact, IQ tests have been found to have correlation coefficients between .5 and .6 with academic achievement and become more accurate as students get older (Brody, 1997). 
Intelligence Quotient tests are offered in numerous languages to better serve the test taker as well. Research has indicated, however, that IQ scores are often lower for minority groups (Rushton \& Jensen, 2003) and those of a low socio-economic status, and do not necessarily gauge intelligence in an effective manner (Ceci, Rosenblum, \& Kumpf, 1998). On the basis of the communication style and differing types of knowledge that are stressed by different cultures, IQ tests can be biased against students with these different communication styles and knowledge bases than the test's authors (Sternberg, 2002).

The SAT is a college admissions test, generally administered in the $11^{\text {th }}$ or $12^{\text {th }}$ grade, used to predict academic achievement in college and it is often correlated to a student's freshman grade point average (fGPA). The SAT scores were found to be able to predict fGPA with $23-30 \%$ accuracy, independently of the College Board that is responsible for creating and administering the test (McDonald et al., 2001). The College Board itself reports higher predictability up to $47 \%$ (Sackett, Kuncel, Arneson, Cooper, \& Waters, 2009). However, the SAT, like the IQ tests, is seen as having biases, including cultural and linguistic ones, resulting in differing predictability rates of college academic achievement among different races (McDonald et al., 2001). The SAT has also been shown to underestimate academic achievement in college by women, demonstrating a predictive bias (McDonald et al., 2001). These biases may exist because of the nature of the tests. For instance, the verbal and writing portions are completely dependent upon the use of language, and the math portion still uses language as well as American mathematic notations to support the mathematical procedures. The biases may be partially attributed to differences in expectations between males and females (Twist \& Sainsbury, 2009). Additionally, the content has been changed to include the measurement of "developed 
reasoning" dependent on content that a student should have been taught in prior academic years as part of the American educational system, making it an unfit predictor for students unfamiliar with the American education system (Zwick \& Himelfarb, 2014, p. 2).

Independent school admission officers, recognizing their need for a common test amongst private schools that could predict academic success, came together in 1957. Collaboratively, they formed the SSATB and worked with Educational Testing Services (ETS) to create the Secondary School Assessment Test (SSAT). The ETS and the SSAB suggest that a high correlation exists between the SSAT and the SAT to demonstrate its validity as well as reliability in predicting academic achievement. They have not, however, published this correlation or the correlation between the SSAT and academic achievement (SSATB: Home, n.d.). Like the SAT, the SSAT is a test that depends on familiarity with not only the English language, but also a familiarity with an American education reflecting bias against students who are unfamiliar with either (SSATB: Home, n.d.).

Consequently, private schools and admissions officers who desire to increase the diversity in their schools should reconsider the use of IQ tests, the SSAT, and other similar tests in light of their recognized biases.

Tests that may limit some of the aforementioned bias are nonverbal aptitude tests. ETS has identified nonverbal tests as being useful for testing students whose primary language is not English to reduce bias (Nonverbal Aptitude, 1993). Nonverbal tests are already utilized in numerous schools to identify students for gifted programs (Giessman, Gambrell, \& Stebbins, 2013). Nonverbal tests, however, have not been fully evaluated to 
determine fairness in their use for admissions (Lakin \& Lai, 2012). A nonverbal test should be identified and studied for use in private schools as part of the admissions process for secondary students in order to help predict academic achievement, while at the same time reducing recognized biases in the testing process.

Two of the better-known tests that have attempted to meet this need are the Wechsler Intelligence Scale (WIS) and Raven's Progressive Matrices (RPM). A recent test that is not as well known is the Naglieri Nonverbal Aptitude Test (NNAT), now in its second edition. When looking at the nonverbal components of these tests, it may be seen that they attempt to assess general intelligence in a similar manner. They utilize matrices or geometric patterns to gauge general aptitude. They begin by presenting problems or sequences that gradually become much more complex as one moves through the test (Mackintosh \& Bennett, 2005; Naglieri, 2006; Wechsler, 2003). Despite the fact that the tests seek to limit the language being used to communicate, the logical skills a student must use to complete the test are comprehensive (Naglieri, 2006).

Two NNAT-like questions are shown here in Figure 1 to give the reader an idea of what a nonverbal problem may look like. They are used by permission of Testpreponline.com in this dissertation (see Appendix C). It is important to note that these problems may be designed for students from 4 years of age through adulthood. The problems may present very basic problems to very complex problems that build on spatial awareness, pattern recognition, or even facilitate the need for complex arithmetic operations that need to be done to solve the patterns. 
Figure 1. Naglieri Nonverbal Aptitude Test Like Problems. This figure illustrates problems similar to those found on the NNAT provided by Test-Prep Online (n.d.).
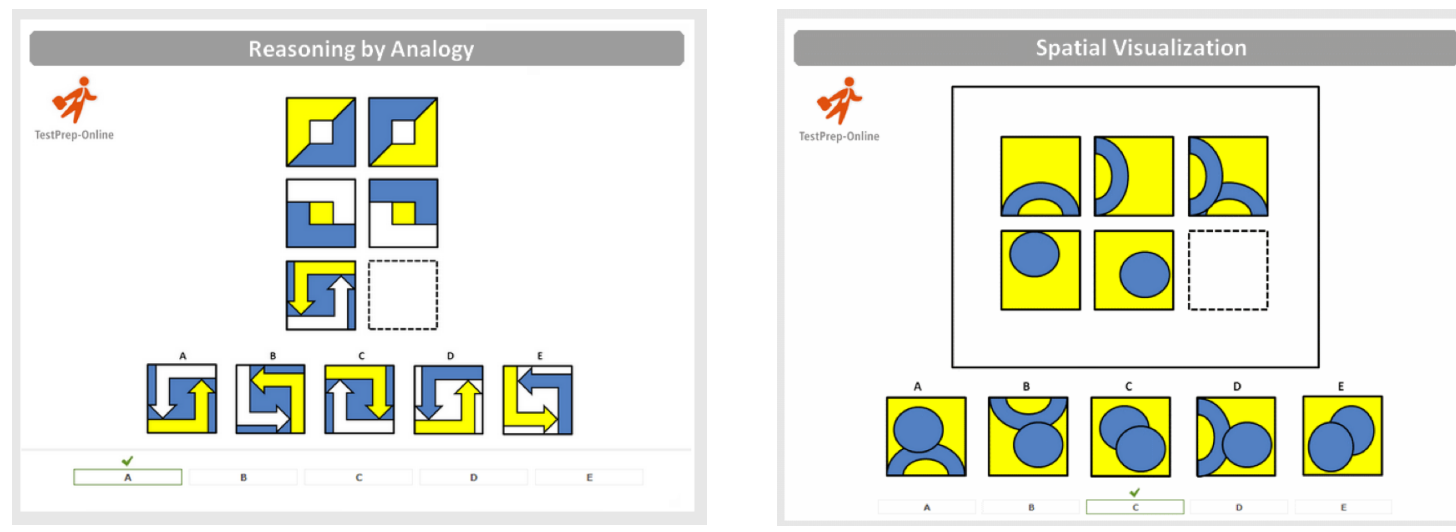

Looking at one example of these tests, the NNAT, we are told that the problems presented test logical thinking as well as spatial and pattern awareness. When student results on the NNAT, given as the Nonverbal Aptitude Index (NAI), are compared to the Stanford Achievement Test, Reading Achievement sub score, a verbal test, in Grades K-9, an average correlation of .56 is identified and recognized as being substantial (Cohen, 1988), with the greatest variance found from grade $\mathrm{K}$ through 4 . The scores were much more consistent, however, in grades 5-9 (Naglieri \& Ronning, 2000). Lakin and Lai (2012) tell us that multi-dimensional tests that include linguistic and mathematical reasoning are better at predicting academic achievement because nonverbal tests do not specifically test language or mathematical skills. These same multidimensional tests, though, also show greater discrepancies between English language learners and native English speakers. Because they show greater discrepancies between English language learners and native English speakers, they may not support diversity in the applications process and do not speak to the students' ability to learn. For this reason, 
multi-dimensional tests are an invaluable tool for diagnostics, but not for initial screening of the students in the application process.

The Wechsler Intelligence Scale attempts to downplay cultural bias by testing four areas: verbal reasoning, perceptual reasoning, working memory, and processing speed (Verney et al., 2005). Language does not encompass the entire test, and the developers worked to reduce its use in most components of the overall test (Wechsler, 2003). Despite this downplay of language, it does still rely upon it and may put students of different cultures at a disadvantage in the schools and programs to which they are applying. In fact, when looking at differential validity between ethnic lines, it is shown to be a much less valid measurement of cognitive ability for Mexican Americans as opposed to White Americans (Verney et al., 2005).

Today, Raven's Progressive Matrices is owned and sold by Pearson. When visiting their website to learn more about it, the author found that it is now marketed to employers to identify those with "talent" who may be trained for higher level work in the marketplace. In other words, it is no longer geared toward educational settings; this skews its results in academic settings as its norms are no longer current or valid in academia, nor have its norms been updated for students. Current norms are verified by reviewing the Norms Tables for their standard and advanced tests where the norms are broken down by corporate positions rather than by age for students (Raven's Progressive Matrices [APM] \& Raven's Progressive Matrices [RPM]).

Ultimately, while the previous two tests, the WIS and RPM, work to reduce bias, they possess drawbacks. The WIS still relies heavily on the English language and RPM's norms have not been updated for students in some time. Additionally, RPM was initially 
developed to test genetic impact on intelligence or the $g$ factor (Mackintosh \& Bennett, 2005).

The purpose of aligning the items with the objectives for which a test is created is at the center of all that is done in the creation of a test and would help to establish the expected range of knowledge, skills, and abilities assessed. If a test is repurposed, it is being misused unless proper validation is done for its new use. If validation is not done, the validity of the test for the new purpose is unknown. Furthermore, assessment providers may be encouraged to imply that their tests are valuable for other purposes when it will in fact increase their sales volume and revenues (Kokhan, 2013). With this in mind, it makes sense to identify an instrument or assessment that was created with the purpose of assessing the cognitive aptitude of diverse students of differing cultures and languages to purposefully reduce bias and usefulness in its application.

\section{Problem Statement}

There is insufficient research and thus a knowledge gap with regards to the link between nonverbal test performance and student achievement. Admissions departments within schools, particularly private schools, often require a predictive assessment to identify a student's potential to find success in a rigorous academic environment (SSATB: Home, n.d.). Numerous tests are available. For the majority, however, student success relies on a high functioning knowledge of the English language. (Lakin \& Lai, 2012). Additionally, a common culture is often assumed by the test creators in regards to the test takers (Greenfield, 1997). With increased mobility of students around the globe, the assumption of a common culture puts many international students at a disadvantage when faced with taking these tests. For this reason, nonverbal test performance should be 
studied in the context of private school settings in order to identify its relationship with academic achievement.

\section{Purpose of the Study}

The purpose of the present study is to explore the relationship between nonverbal test performance and academic achievement. Because previous studies have shown that traditional IQ and aptitude testing may present bias in testing and placing students into appropriate programs (Verney et al., 2005), nonverbal testing may better gauge student aptitude within a diverse population because of the purported reduction of bias in nonverbal testing (Naglieri \& Ford, 2003).

The present study was undertaken to determine whether nonverbal aptitude as measured by a nonverbal aptitude test accurately predicts academic achievement as measured by second semester grade point average (GPA) in a private secondary school setting while controlling for gender, grade level, whether the student is a native English speaker or not, and English as a Second Language (ESL) level. If nonverbal aptitude as measured by a nonverbal aptitude test demonstrates this ability, then it might be seen as fit for admission purposes depending upon the setting and the manner in which it would be used, in conjunction with other indicators as identified by an admissions department. The study also sought to determine whether the mean results were comparable across differing demographic factors to include gender, and whether the student was a native English speaker or not. If a difference across the means was identified in any of these groups, further research may be necessary to assess whether bias exists against any of these groups from the test. 


\section{Research Questions}

1. Is there a relationship between students' nonverbal academic ability and academic achievement, while controlling for gender, grade level, being a non-native English speaker, and ESL level at the secondary grade levels (6-12)?

2. Is there a significant difference in the nonverbal academic ability mean scores of the applicants at the secondary grade levels (6-12) between the genders?

3. Is there a significant difference in academic achievement score means of native English speakers versus the non-native English speakers at the secondary grade levels (612)?

\section{Research Hypotheses}

1. Nonverbal aptitude as measured by the NAI score, the independent variable, accounts for a significant amount of unique variance in predicting academic achievement as measured by the second semester GPA, the dependent variable, when controlling for grade level, gender, primary language spoken, and ESL level as measured by WIDA at the secondary grade levels (6-12).

2. There is a significant difference in nonverbal aptitude mean scores as measured by the NAI score at the secondary grade levels (6-12) by or between the genders.

3. There is a significant difference in nonverbal aptitude mean scores as measured by the NAI score of non-native English speakers as compared to native speakers at the secondary grade levels (6-12).

\section{Theoretical Framework}

The admissions process should be one that provides students the ability to demonstrate their cognitive aptitude in a manner that neither their cultural diversity or 
gender negatively affects their opportunity if they holistically have the ability and desire to challenge themselves in a rigorous curriculum at a given school. To best understand the proposed study and its intent, three ideas must be understood: the concept of aptitude expressed in this study, the relationship between nonverbal aptitude and aptitude, as well as Bandura's social cognitive theory to understand why the second semester GPA was used for a student rather than the first semester or the cumulative first year GPA.

As humans grow and develop, cognitive intelligences take shape, as do different behaviors. These intelligences may be reinforced and may change through experience and training (Seligman, 2007). Psychologists have analyzed growth and development for years in order to discover the influences that shape an individual. The analysis of growth and development has led to a number of debates: the nature vs. nurture debate (Pajares, 2002), and entity theorists vs. incremental theorists (Furnham, 2014). Psychologists, however, are finding that both nature and nurture play a role in shaping a person (Pajares, 2002). Additionally, the ability to increase an intelligence may be determined by the specific intelligence or construct that is isolated by a psychologist for study, whereas some intelligences can be increased and others may be static (Furnham, 2014). Furthermore, Dweck (2010) has told us in her mind set theory that building intelligence may also be dependent on people's mindset and their belief in whether they themselves can build intelligence.

These arguments may also influence how one perceives aptitude. A common synonym for the word aptitude is potential. If aptitude is perceived as potential, then there is a need to quantify attributes of nature, or inherited ability rather than those that have been instilled in students through training. If a student has the inherent ability to 
learn at a high level, then current academic achievement, nurture, should not preclude a student from future high-level learning. Additionally, inherent ability can make up for a past deficit or difference often identified as an achievement gap that may exist as a consequence of other extenuating circumstances.

Achievement gaps are recognized in differing academic areas such as math or reading. Numerous causes are recognized for contributing to these gaps in education, such as socioeconomic status as well and language challenges. These gaps are often identified through the use of standardized testing (Anderson, Medrich, \& Fowler, 2007). For this reason, the gaps that are found within academic areas identified by the test creators often reflect the majority's opinion of what is important within a culture. These achievement gaps, therefore, may not be reflective of aptitude.

The development of nonverbal testing found its roots in a practical purpose. The United States military found a need during World War I to test large groups of recruits at a time who were either illiterate or had low literacy levels. This was necessary to appropriately place these recruits into positions or jobs where they had the aptitude or potential to do well regardless of their literacy level (McCallum, Bracken, \& Wasserman, 2001).

The development of tools for this purpose has led to their application with numerous populations. Nonverbal testing is now used with populations such as individuals with hearing loss and with individuals with learning disabilities (DeThome \& Schaefer, 2004).

Carroll (1993) sought to better understand intelligences and aptitude or ability. He performed a study of numerous data sets that led him to build upon prior theory and 
divide intelligence into three stratums: the $g$ factor, broad ability, and narrow ability. The $g$ factor is an overarching factor or ability that plays into all intelligences; the broad abilities are groupings of related narrow abilities similar to Gardner's (1983) theory of multiple intelligences.

Horn and Cattell (1966) developed a theory of general intelligence as well. When looking at general intelligence or the $g$ factor, they theorized that it had two discrete components: fluid and crystallized intelligence. Fluid intelligence allows one to solve novel problems and include the abilities to recognize patterns, reason abstractly, and problem solve. Crystallized intelligence is shown in developed skills such as language. Because these skills can be developed, this intelligence can be expanded. The theory tells us the students with a higher fluid intelligence have a tendency to gain crystallized intelligence at a faster rate.

McGrew and Flanagan (1998) took these two theories to develop the CattellHorn-Carroll theory. They theorized that verbal IQ represents a construct of crystallized intelligence. They further theorized that nonverbal IQ is not in and of itself a construct. Nonverbal IQ is, in fact, the demonstration of abilities expressed nonverbally and may represent numerous constructs of fluid intelligence.

Naglieri (2006) stated that verbal and nonverbal distinctions in tests refer to the transmission of the ideas involved, not the type of thinking or intelligence required. For this reason, verbal and nonverbal tests are nothing more than differing ways to measure general ability. Looking back to the Cattell-Horn-Carroll theory, one may see that the nonverbal tests may more definitively test fluid intelligence. High fluid intelligence ability is theorized to enable a student to build crystallized intelligence more quickly 
(Horn \& Cattell, 1966). Moreover, a nonverbal test that challenges students to think in multiple ways to solve progressively harder problems should theoretically be able to ultimately predict academic achievement if a student is given time and support to succeed in a new academic environment. Thus, the distinction between verbal and nonverbal tests is the ability of the nonverbal test to remove bias potentially found in language or culture.

This idea, that the distinction in testing is the ability to remove bias, directly shapes the work of psychologists in the development of testing material, such as found in the work of Naglieri. His work in this field has led to the development of the NNAT (Naglieri \& Ford, 2005). When reviewing the literature for the NNAT as provided by Pearson, one sees that its intended purpose is to remove bias that exists in other forms of testing (“Technical Information,” 2011). These biases may concern language, culture, socioeconomic status, gender, etc. (Naglieri \& Ford, 2005).

The novelty in Naglieri's NNAT and his approach to gauging aptitude then may be the identification of general aptitude while reducing bias attributed to language and cultural differences as well as differences attributed to gender. This method of testing then works to identify personal natural abilities without associating it with a particular race or gender and not preserving racial stereotypes that more recent testing has shown to be partially attributable to testing bias (Neisser et al., 1996; Jensen, 1980).

Lohman, another test developer, and developer of the Cognitive Abilities Test $(\operatorname{Cog} \mathrm{AT})$, is a proponent of tests that also include a verbal and quantitative component. $\mathrm{He}$, too, sees the merit of the nonverbal component, but argues that the measure is a better predictor of future academic success (aptitude or potential) because of its inclusion of not only nonverbal components, but also verbal and quantitative batteries. His 
rationale is that prior academic achievement is a better predictor of future academic achievement than aptitude in isolation (Lohman, 2005). In his argument, his definition of aptitude clearly includes prior academic success. The challenge in Lohman's definition, however, is that prior academic success is in the culture of the majority. The culture of the majority may utilize language, mathematical notation, or other cultural differences in creating a test that is ultimately to the detriment of the minority or culturally diverse student in admissions testing (Verney et al., 2005).

Social cognitive theory (SCT) stands in contrast to developmental ideas that emphasize either nature or nurture completely over the other (Bandura, 2002). Bandura has not seen humans as merely reactionaries to stimuli in the world. Rather, he has posited that humans organize their thoughts, are active in their development, and that through self-reflection and self-regulation, they are able to adapt to environmental stimulus to control the environment they live within.

An individual's acquisition of knowledge is directly related to the observation of others in a social context that includes interactions and experience. When one observes a model of behavior, as well as the consequence to that behavior, one will make an innate decision on whether that modeled behavior is of value in one's personal life. The innate decision that is made will directly affect learning because it helps a person to value individual behaviors. The value placed on individual behaviors may encourage a person to replicate what is witnessed, or even to avoid it. New behaviors are not adopted by either success or failure alone, but by the perceived social or experiential value it adds to oneself (Bandura, 2002). Given the SCT and its implications concerning learning, one 
can predict that students with an aptitude for high academic achievement placed into a rigorous program will learn to be successful.

Students will replicate what Bandura (2002) sees as "triadic reciprocality" (p. 24) in their personal worlds, in which personal factors such as cognition and biological traits can and will affect behavior. Triadic reciprocality in turn can influence and affect the environment one lives within, shaping future cognition and self-regulatory actions and creating a triangular pattern in which these three concepts constantly influence one another (Pajares, 2002).

Based upon the interaction of these three concepts, one can conclude that there are agents of change involved in human development. Personal agents are those agents that one exercises individually to encourage change in one's self. There are also proxy agents, or other parties involved in one's life that encourage change. In the student's setting, the proxy agent may be the school or program that he or she has entered. The last agent of change is known as collective agency, in which one interacts with others to collectively cause change (Bandura, 2002).

The inclusion of triadic reciprocality, as well as the three agents for change in SCT, takes into account all variables in one's life to see how growth and development occur. While individual growth is monitored, it is recognized that it is more than the individual that promotes growth. Factors in one's life such as socioeconomic status, education, family, faith, etc. all play a role in fostering personal development. All of the aforementioned factors, however, must still be cognitively analyzed by an individual before they affect him or her and are thus indirect (proxy) agents of change (Pajares, 2002). 
Humans have varying abilities to symbolize, strategize, learn through observation, self-reflect, and self-regulate. Certain cultures may emphasize one or more of these abilities. These abilities are what make one human, and can influence the environment around one's self. Bandura (2002) considers self-reflection to be the most important of these, as it allows personal conclusions to be drawn, to include where one sees oneself within one's environment. This will also lead one to pass personal judgment that will affect future performance (Bandura, 2002).

As schooling also provides a social education, the environment includes additional agents such as peer groups that can influence a student's development. This may be through the direct encouragement of peers, or also through observational learning. A student does not have to directly experience outcomes, but can learn vicariously through others (Bandura, 1989).

Following this concept, motivation is then also seen as dependent upon on a number of agents. High levels of motivation in accompaniment with a natural aptitude then lends itself to academic success. Bandura (1997) and Zimmerman (2000) have told us that self-efficacy is a personal judgment of one's own ability to take action to successfully complete tasks and goals and is seen as directly impacting one's persistence to achieve success. In this model then, measuring aptitude becomes important, realizing that motivation is an orientation that may be learned by providing an environment in which a student's self-efficacy is re-enforced with peers who provide a good example to follow, along with positive interaction and feedback to promote a positive environment (Burney, 2008). 
The development that takes place within a child or adult is not strictly cognitive. Social development, moral development, as well as motivation development may all take place. The changes that take place may be the result of direct, observational, vicarious experiences of consequences, or because of modeling by others within the student's environment. In the individual's development, strategies to face differing challenges presented to the student will occur and as learning and development take place, these strategies will become more advanced (Bandura, 1989).

Given SCT, one would anticipate a student with the aptitude to master a rigorous curriculum would find success within an environment that is supportive of that goal and provides encouragement. By identifying aptitude through an unbiased approach in the admissions process, the benefits of a rigorous curriculum can be offered to a diverse student population that reflects the community and not one that favors the American majority as many tests do (Giessman et al., 2013), allowing that diverse student population the ability to take advantage of a more challenging education. Conversely, when little support is offered within a curriculum, a diverse student body may be less likely to find success. When the SAT is used to predict academic success at the university level, it is known to be most accurate when looking at the freshman GPA. Each subsequent year of study proves the SAT less reliable (Elert, 1992). The reduction in reliability over time may be a result of numerous factors, such as the freedom a student has on campus, the support networks that exist, or even expectations in education from the home. Evidently, beyond initial placement, the environment that a student is placed into plays an important role in development and academic achievement (Elert, 1992). 


\section{Overview of Methodology}

To determine the ability of nonverbal aptitude to predict academic achievement, an ex post facto study was conducted at a private secondary school (6-12). It was an ex post facto study with hypotheses that controlled for viable alternative explanations. This type of ex post facto research is seen as the most powerful (Kerlinger \& Lee, 2000; Newman, Newman, Brown, \& McNeely,, 2006). A nonverbal test was identified, the Naglieri Nonverbal Aptitude Test II, to index the construct. Data on new students were collected for a three year period to obtain as large a sample size as possible. A correlational analysis was then conducted to identify whether a positive relationship existed between the results of the nonverbal test, the NAI score prospective students obtain, and the GPA they earned during their second semester at the school. Differences of the mean NAI scores between male and female students as well as between native and non-native English speakers were analyzed to identify whether bias was evidenced in the test.

\section{Delimitations of the Study}

The researcher chose to perform his research at a single private school in South Florida. This study required NNAT testing to be incorporated into the admissions process of the school, and for this reason, other schools that were contacted were not interested in participating in this study. Additionally, the researcher chose to focus this study on students in the secondary grade levels. This choice was made as there is limited research at these grade levels in regards to nonverbal testing. Three years of data is being utilized as this study is an ex post fact study, and these were the years with data available. 


\section{Threats to Internal and External Validity}

The design of this research resulted in weak internal validity because the research is ex post facto. Ex post facto research can only identify potential inferences, but it cannot have causal inferences (Newman et al., 2006). Causal cannot be identified in correlational studies, however, trends can be identified that can be explored with a robust experimental design in the future.

External threats to the validity of this study existed. Students who were enrolled at the school did leave the school before the end of their first year, reducing the sample size. Additionally, it was assumed that the desire to be enrolled in the school as well as to do well in school would encourage students to do the best they could both on the nonverbal aptitude test as well as academically. Students who did not perform to the best of their ability or who purposefully do poorly either on the aptitude test or in their coursework threatened the validity of this study by taking away from response validity. Unfortunately, motivational factors cannot be statistically controlled for in this study as this information is not available for this ex post facto study.

Additionally, while the population within the school that was the subject of this study serves students from numerous countries all over the world, the majority of students are from Latin America. The school itself is a religious school, and this may also limit its applicant pool. For these reason population, validity may be limited and make it more difficult to generalize the findings to other groups.

\section{Assumptions}

In conducting this research, some assumptions were made. It was assumed that the need for supporting a diverse student body would increase, considering the fact that 
immigration is an ongoing occurrence and these students will need an education. The U.S. Department of Education projected a decreasing number of both Black and White students through 2022 and an increasing number of Hispanic and Asian students (Projections of Education, 2014). A second assumption that was made was that private schools will actively work to meet these students' needs. If private schools do not work to address their needs, then they will continue to miss a significant market. These two assumptions created the need to further study the link between nonverbal test performance and academic achievement in the setting of a private school.

When students apply to a private school, another assumption was made.

Admission officers will generally anticipate that the student has a desire to attend the school or is motivated to do well. This may not always be the case as the student may be forced to test by a parent who wants their child enrolled in the school. In order for this study to be the most accurate, the assumption was made then that the students are doing the best they can on the placement test.

\section{Definition of Key Terms - Operational}

Academic achievement - the level of success a student has achieved in school as calculated by second semester Grade Point Average.

Admissions - those in a school responsible for testing and interviewing a student to determine their acceptance into a school.

Aptitude - in this research, aptitude refers to a student's potential rather than prior learned skills, as measured by the Naglieri Nonverbal Aptitude Test and given as the Nonverbal Aptitude Index. 
Cognitive Ability Test (CogAT) - a test in its sixth edition written by lead author David Lohman and Elizabeth Hagen and published in 2001 that uses a battery of quantitative, verbal, and nonverbal items to gauge a student's general aptitude (Cognitive Abilities Test, n.d.).

College Board - Developers of the SAT.

Educational Testing Services (ETS) - the world's largest non-profit educational testing and assessment organization.

ESL Level - ESL levels range from one, entering; to six, reaching, according to WIDA. These values may be seen as being representative of a student's ability in the use and comprehension of language ranging from a beginner to native level user of the English language. The values, while continuous, are not seen as interval data but as ordinal values as each decimal represents a proportion within a proficiency level and not seen as equivalent to a decimal place within another proficiency level (ACCESS for ELLS, 2011).

$g$ factor - An idea presented by Charles Spearman that states a person that performs at one level on one particular cognitive task generally performs similarly on other cognitive tasks (Lovie, 2005).

Gifted programs - curricula established within schools that serve students that are seen as especially talented.

Grade Point Average (GPA) - the GPA is the numerical average of a student's earned grades in a particular term, the second semester of a new student's first year in this study. The Grade Point Average is calculated using a 4.0 scale that awards an 
additional .5 points to each class that is considered advanced by the school such as Advanced Placement (AP) classes.

Independent Variable - in this research, this term is utilized frequently and interchangeably with predictive variable. An independent variable may infer causation, whereas a predictive variable does not. The use of this term is not to infer causation. The independent variables utilized in this study include academic achievement, grade level, ESL level, gender, GPA, and whether a student is a native English speaker.

Intelligence - a construct that attempts to identify a person's intellectual performance in given situations and gauged by different means such as standardized tests. General intelligence paints this construct with a broad stroke, whereas other constructs of intelligence may be defined much more narrowly (Neisser et al., 1996).

Intelligence Quotient Test (IQ test) - one of many tests designed to assess human reasoning ability measured through problem solving. The results are compared to a statistical norm or average that is set at 100 .

Minority students - Students who reflect smaller sub populations within the United States as opposed to the majority, such as Black, Hispanic, or Asian.

Nonverbal Aptitude Index (NAI) - the scale used to identify the results of the NNAT, where 100 is set as the mean score similar to a traditional IQ test.

Naglieri Nonverbal Aptitude Test (NNAT) - a test utilized to gauge cognitive ability or aptitude apart from language and cultural background using progressive matrices.

Nonverbal Test - tests gauge academic aptitude based upon problem solving problems that avoid the use of language. 
Potential - the capacity to develop skills or build upon intelligence in the future.

Raven's Progressive Matrices - a test developed by John Raven in 1936 that was created to gauge general intelligence using progressive matrices, initially intended for school-aged children (Raven, 1936). It is now primarily used by employers to test employees for promotion (Raven's Progressive Matrices [APM], 2012).

Scholastic Assessment Test (SAT) - a college admissions test generally taken in the $11^{\text {th }}$ grade.

Self-efficacy - the perception of the likelihood of one's own ability to reach a goal or complete a task.

Stanford Achievement Test 9 (SAT9) - The SAT is a comprehensive set of tests that are given at each grade level from Kindergarten through the $12^{\text {th }}$ grade. It tests reading comprehensions, mathematical problem solving, language, spelling, listening comprehension, science, and social studies. A norm-referenced test tests for academic knowledge at each grade level.

Standardized Test - a comprehensive test given to a student intended to estimate a student's learned reasoning and problem solving ability or to predict a student's aptitude compared to other students of similar age or grade using "norms" established through prior studies.

Secondary School Assessment Test (SSAT) - an admission test given in many private schools in Grades 3 to 11 to offer a standard measure to determine admission into a school. 
Secondary School Assessment Test Board (SSATB) - an organization formed of independent school admission officers to support each other and to create an admission test to help in determining acceptance of new students into private schools.

Wechsler Intelligence Scale (WIS) - a test developed by David Wechsler first released in 1949 and currently in its fifth edition, to test humans across differing intelligences producing differing scores based upon problem type to include verbal, perception, working memory, and processing scores in contrast to an IQ test that produces one score (Kaplan \& Saccuzzo, 2009).

\section{Significance of the Study}

The case for the need for unbiased testing in academic settings has been highlighted by the disproportionately low number of non-White and Hispanic students enrolled in high academic level courses and programs (Naglieri \& Ford, 2003). Theorists such as Bandura (2002) tell us that people who find themselves in social situations that positively encourage success will be more likely to succeed. These ideas, coupled with the use of intelligence tests to predict academic success (Watkins, Lei, \& Canivez, 2007), necessitate the need to identify tests that are unbiased in gauging intelligence to place more diverse students into challenging courses and programs where they can find success.

Nonverbal tests have been identified as a possible means to gauge student intelligence in a manner that reduces bias (Lewis, 2001). Research has been done in which nonverbal aptitude testing was correlated to other aptitude tests and/or standardized tests for purposes of validation (Naglieri \& Ronning, 2000). Empirical research needs to be done, however, that determines whether there is a correlation 
between nonverbal aptitude tests and academic achievement as identified by earned grades through students' GPA. This is one of the first studies that looked at the predictive validity of a nonverbal aptitude testing for predicting academic success as identified by GPA earned rather than its correlations with other tests such as the SAT-9. Furthermore, it is also one of the first studies that examined the validity of nonverbal aptitude testing for secondary students to predict academic achievement as identified by GPA. Because it is one of the first studies to determine the predictability of general academic achievement as measured by GPA by a nonverbal aptitude test, it provides new data in this area showing the relationship that exists between academic aptitude and academic achievement as demonstrated in numerous subject areas through the students' GPA.

This study further contributes to the existing body of knowledge by demonstrating the ability that a nonverbal aptitude testing has in predicting academic success, despite potential barriers such as language as indicated by Naglieri (2006). By looking at students' GPA, this study correlates aptitude with academic achievement collectively quantified over numerous subject areas.

The admissions department within a school may also want to consider the findings of this study. If a significant positive correlation is identified, it substantiates the inclusion of nonverbal testing as a key component in placing students into rigorous courses and programs in order to reduce recognized biases found in other tests that rely upon language or other means that may present bias. This would allow school to accept a more proportional number of minority students into these programs to better represent the demographics of its applicant pool within a given school. 


\section{Chapter Summary}

Students applying to schools have varied backgrounds. These backgrounds may present differences in language, customs, cultures, etc. from the White non-Hispanic majority. The differences are not indicative necessarily of cognitive aptitude. Despite this fact, standardized testing used to identify a student's aptitude is often based on both language and assumed similarities in culture to the test creators.

Research has shown us that numerous factors play a role in the academic achievement that a student achieves. One important predictor is general intelligence. Naglieri (2006) theorizes that nonverbal intelligence as a measure of general intelligence can predict academic achievement similarly to other forms of intelligence tests. Empirical research has correlated intelligence to academic achievement as measured by other tests.

Admissions officers in schools need to assess its applicants' ability in order to accept students and to then place them into appropriate programs. One method of gauging academic aptitude, while reducing bias toward differing groups of students, may be to utilize a nonverbal aptitude test in which language and culture do not present themselves in the test.

If a nonverbal aptitude test score can accurately predict academic achievement, it could be utilized as a tool in the admissions process to complement other tools that are utilized to best accept and place students into appropriate programs.

\section{Organization of the Study}

The remainder of the dissertation will look at the theoretical orientation of this study, the methodology that will be utilized in this study, as well as the results. The 
results will be discussed as to how they may be applied in application as well as what gaps are left by this study for future research. 


\section{CHAPTER II}

\section{REVIEW AND CRITIQUE OF THE LITERATURE}

To best understand this study, its theoretical orientation needs to be discussed. Connecting nonverbal aptitude to academic achievement in an educational setting ties together numerous theories to support making the predictions, interpreting the data, and making sense of theoretical, empirical and practical implications related to the findings of this study. Bandura's (2002) SCT must be understood to best understand how a new student in a new academic environment may react. The construct, nonverbal aptitude, needs to be discussed as well as the theories that support its use in gauging intelligence and its link to learning and academic achievement.

Additionally, prior academic reviews of nonverbal aptitude testing must be reviewed before performing this study to understand the arguments for and against its use in the screening process. When looking at each side, it will become apparent that one's definition of aptitude will determine the framing of the results. To become more inclusive of community diversity, it becomes evident that biased testing needs to be replaced by that testing designed to minimize it. After addressing the two sides in this argument, the need for further review research as well as for this study will present itself.

An extensive survey had to be done to identify relevant resources to contribute to this dissertation. Nonverbal aptitude and its testing had to be researched as well as the Bandura's SCT. Recent research of nonverbal aptitude testing frequently identified the Wechsler, the CogAT, and NNAT as among the most common tests used in this field today. The NNAT was the only one, however, that exclusively used nonverbal components in its testing battery. The Buros Center for Testing was referenced for 
information on the NNAT2 specifically, but due to its recent publication date, they do not provide information concerning it yet. In searching for articles associated with nonverbal testing, searches were carried out for Nonverbal Aptitude Test, Naglieri, Wechsler, Raven's Matrices, Aptitude Testing, Nonverbal Intelligence, Multiple Intelligences, Predicting Academic, Academic Placement, and combinations of these key words. When searching for work on Bandura's social cognitive theory, Bandura and social cognitive theory were searched for as well.

ERIC, ProQuest, PsycArticles, PsycINFO, and Psychiatry Online were all databases that were referenced through the Florida International University Library to find additional resources pertaining to the research topics.

\section{Theoretical Orientation}

Students do not just react to the world around them. Instead, they receive stimuli, organize their thoughts around the stimulus, and then react. The student may then receive both internal and external feedback as a consequence to their actions. Internal feedback may include emotional responses, whereas external feedback may include the reaction of teachers, peers, parent, etc. The student may then learn from the stimulus they were presented with as well as the consequences of their actions. This forms the basis of Bandura's social cognitive theory (SCT). SCT acknowledges that social interaction plays a significant role in shaping who one becomes as well as their individual intelligence or aptitude that impacts cognition. People learn by observing others. One's own intelligence will play an integral role in the decision making process as to how one interprets observed behavior as well as the social reactions to the given behavior (Bandura, 2002). 
Bandura's work seeks to incorporate a triadic reciprocity in understanding how one learns, to take into account all variables in one's life to see how growth and development occur. A reciprocal relationship is formed by the individual to include factors in one's life such as socioeconomic status, education, family, faith, etc.; the environment one is in and can include the home, school, and social groups, etc.; as well as the cognition that takes place within an individual to create order out of it all (selfreflection). Each of these variables can influence all of the others, and all play a role in fostering growth (Pajares, 2002).

SCT is an important underlying concept in this research as the students' second semester GPA was used to measure academic achievement. This was done purposefully as new students, regardless of background, were introduced to a new school. This included new teachers, new expectations, new peer influences, possibly a new language, etc. All of these factors, to include the students' own expectations, could play an integral part in their growth and development, according to SCT, and affect their overall performance. SCT shows us that grades reflect this new social setting as a person's continued reaction to stimulus is based upon both internal and external factors.

SCT provides the rationale as to why this study uses the second semester GPA and not the first. It allows students time to begin learning the new social expectations, as well as academic expectations. It allows the students to begin familiarizing themselves with a new language and gives them time to react appropriately. This would allow aptitude testing to be more accurate, as the student can now work to meet known expectations with the support provided in the new environment. 
Duckworth (2006) researched IQ, self-discipline, and grit to identify the best predictor of academic achievement for students within a given school year. In this study, she also utilized spring or end of the year grades in her study as it allowed students to make choices in their behavior based upon the received stimulus in response to their performance earlier on in the year. She found that even when controlling for first semester grades, these indicators were significant in predicting end of the year grades, demonstrating that first semester grades and end of the year grades will vary as students adapt to the expectations of a new grade level.

While one's social surrounding and one's reaction to it may do as much to promote academic achievement as one's general intelligence according to SCT, intelligence cannot be ignored. The impact of SCT on a person's performance can cause a researcher to reevaluate traditional theory. Some scholars have asked whether intelligence predicts academic achievement, or does academic achievement predict intelligence (Watkins et al., 2007). Deary, Strand, Smith, and Fernandes (2007) performed a five-year longitudinal study of over 70,000 English students to examine whether intelligence can be used to predict academic achievement. Intelligence was measured at age 11 and correlated to achievement in over 25 subject areas at the age of 16. The researchers found that with math and English, a correlation of between $48 \%$ and 58.6\% was identified; whereas in Art and Design, there was a lower correlation of $18.1 \%$. In every academic subject, however, general intelligence contributed to success. Watkins et al. (2007) further substantiated this claim by doing a cross-lagged panel analysis of IQ testing and achievement testing utilizing a test-retest period of 2.8 years. The authors found IQ did have a moderate ability in predicting achievement, .466 (Cohen, 1998), and 
that the results of the ability of achievement tests to predict IQ were not significant (Watkins et al., 2007). These results are indicative of the ability intelligence has in predicting academic achievement.

Naglieri (2006) tells us that information is taken in through multiple means to include both verbal and nonverbal stimuli. This takes place on tests, as well as in life. This external stimulus does not dictate how a person internally processes the information. That being said, Naglieri then believes that aptitude testing can be done nonverbally.

This builds upon the Cattell-Horn-Carroll (CTH) theory that sees general intelligence, or the $g$ factor, being comprised of both fluid and crystallized intelligence (McGrew \& Flanagan, 1998). Fluid intelligence is the ability to solve novel problems that does not necessarily rely on crystalized or learned knowledge completely. A high fluid intelligence allows one to utilize all means available in one's environment, to include one's crystallized knowledge to solve problems and build new knowledge. This high fluid intelligence may contribute to one's ability to build crystallized knowledge more quickly and allow a student to be successful in a rigorous academic program. A nonverbal test is designed to test general aptitude or the $g$ factor without the use of verbal language (Nonverbal Aptitude, 1993). Based upon CTH theory, one would expect that a student who does well on a nonverbal aptitude test has a high fluid intelligence as they are able to solve novel problems both accurately and quickly as the tests are timed. The theory tells us that students with a higher fluid intelligence have a tendency to gain crystallized intelligence at a faster rate. Crystallized intelligence is shown in developed skills such as language. Because these skills can be developed, this intelligence can be expanded. By raising one's crystallized intelligence and having a higher fluid 
intelligence, a student will be able to use the newly acquired knowledge to find academic success in an academic environment.

Naglieri (2006) tells us that verbal and nonverbal distinctions in tests refer to the transmission of the ideas involved, not the type of thinking, or intelligence required. For this reason, verbal and nonverbal tests are nothing more than differing ways in which to measure general ability. Looking back to CTH theory, one may see that the nonverbal tests may more definitively test fluid intelligence. High fluid intelligence ability is theorized to enable a student to build crystallized intelligence more quickly (Horn \& Cattell, 1966).

A nonverbal test then, which in itself is novel for students, challenges them to think in multiple ways to solve progressively harder problems, should then be able to ultimately predict academic achievement. This fluid intelligence allows a student the ability to build crystallized intelligence more quickly. If students with these inherent abilities is given time and support to succeed in a new academic environment, they will more likely do so.

This idea, that the distinction in testing is the ability to remove bias, directly shapes the work of psychologists in the development of testing material, such as found in the work of Dr. Naglieri. His work in this field has led to the development of the NNAT (Naglieri \& Ford, 2005). When reviewing the literature for the NNAT as provided by Pearson, one sees that its intended purpose is to remove bias that exists from other forms of testing (Technical Information, 2011). These biases may include language, culture, socioeconomic status, gender, etc. (Naglieri \& Ford, 2005). 
The novelty in Naglieri's NNAT and his approach to gauging aptitude then may be the identification of general aptitude while reducing bias attributed to language and cultural differences as well as differences attributed to gender. This method of testing then works to identify personal natural abilities without associating it with a particular race or gender and not preserving racial stereotypes that more recent testing has shown to be partially attributable to testing bias (Neisser et al., 1996; Jensen, 1980).

This is important when identifying a test for this particular application, as there are numerous nonverbal tests available such as the NNAT, the Wechsler, Raven's Progressive Matrices, and the CogAT. Each of these tests were created and are used for a particular reason. Because of this, existing literature must be carefully reviewed as it is frequently reviews nonverbal tests in ways that do not directly apply to admissions. The problematic usage of the words intelligence, aptitude, and ability interchangeably makes the review of literature challenging (Macklem, 1990) because it hinders pinpointing the exact meaning of these words as well as identifying the intended purpose of a particular test or battery of tests.

\section{Literature Review}

Academically rigorous programs seek to challenge students to promote continued growth at high cognitive levels. Students of differing backgrounds may possess high cognitive ability. Due to their differences in backgrounds - whether it be linguistic, cultural, or economic - the diversity of the general population is not reflected in gifted programs within the United States (Naglieri \& Ford, 2003). To address this problem, many gifted programs have adopted nonverbal aptitude testing into their screening process (Lakin \& Lai, 2012). Nonverbal intelligence tests were designed to quantify 
general intelligence, while reducing any bias or inhibition presented by language ability (DeThome \& Schaefer, 2004).

Nonverbal aptitude testing is different from an achievement test as it seeks to identify potential without the use of language, and not gauge prior mastery of skills (Nonverbal Aptitude, 1990). If a student shows high potential, an appropriate education can push that student to achieve high standards. This differs from most aptitude testing because its content is different. For instance, many aptitude tests will include languagebased prompts as well as mathematical components (Nonverbal Aptitude, 1990). Proponents of these tests would argue that prior high academic achievement would allow students to do better on these tests (Lohman, 2005). The difference then is not of aptitude, but definition. Does aptitude include prior mastery? If the answer is yes, then many students who differ from the "majority" will be less likely given a chance to participate in rigorous academic programs (Naglieri \& Ford, 2005).

In an effort to best reach students who traditional tests have been biased against in the past, rigorous academic programs to include private schools may wish to include nonverbal aptitude testing as part of its screening process to become more inclusive of those of diversity within their local communities. This includes ESL students who may have very limited exposure to English. Moving into the future, this trend is expected to increase, and inclusion of these students should not be ignored (Naglieri \& Ford, 2005). As students get older, the programs that are made available to them regularly become increasingly inclusive as seen by the use of standardized tests and their results. The older a student is, the higher the scores must be for inclusion in rigorous programs (Shaunessy, Karnes, \& Cobb, 2004). For this reason, this study seeks to identify the 
correlation between nonverbal aptitude and academic achievement in a secondary private school setting, as expressed by the second semester GPA in a rigorous program, to give the students time to adapt to the school and its expectations keeping in mind Bandura's theory. Additionally, this study looks then to identify whether linguistic or gender bias is minimized by nonverbal aptitude testing based upon the results of the study.

\section{Critique of Previous Research}

The researcher will present a discussion of previous research in regards to nonverbal aptitude testing and its ability to predict academic achievement. Numerous nonverbal aptitude tests are available. Despite this fact, most recent research references but a handful. In an effort to present their validity, most reviews of nonverbal tests correlate one with another. Similarly, few studies identify a correlation between nonverbal aptitude test results and more traditional aptitude or standardized tests such as the SAT-9. None of the studies identify a correlation between the nonverbal aptitude test results and academic achievement as determined by grades or GPA.

Research that focused on a secondary setting was also difficult to find. Indeed, only one study presented any information at a secondary level, and the majority of the sample was found in the elementary grades. The shortage in research at this grade level needs to be addressed by further studies.

The studies presented below identified a correlation between nonverbal aptitude tests and academic achievement (as identified by either GPA or a correlation with other standardized tests such as the SAT-9).

Predicting academic success. Brody (1992) tells us that one of the most important dimensions of validity for an ability test is its ability to predict school 
achievement. If a test is able to do this efficiently, then it provides relevance to the construct that it attempts to gauge. If multiple tests to include verbal and nonverbal correlate well with one another but not with academic success, then the commonality of the construct that is gauged within the tests is of little importance in predicting academic achievement. Considering the knowledge that many constructs such as motivation and family involvement may also have an impact on grades (Allan, 2005), one would look for a positive correlation similarly established in other tests that seek to do a similar thing. The SAT has been found to have from a $23 \%$ to $47 \%$ ability to predict fGPA depending upon the sample (McDonald et al., 2001; Sackett et al., 2009). These effect sizes can be seen as ranging from medium to large, according to Cohen (1988). For this reason, an aptitude test, as previously stated, should be only part of a comprehensive admissions and placement program.

In 2010, Balboni, Naglieri, and Cubelli performed a correlational study in Italy to identify the correlation between both the Raven's Colored Progressive Matrices (CPM) and the NNAT (two nonverbal aptitude tests) with academic achievement in math and reading comprehension as identified by a standardized test established, the Multilevel Academic Survey Test. Students $(N=269)$ in an urban area in Northern Italy were tested. The findings were that scores on the two tests and their ability to predict academic achievement as identified by standardized tests varied between .32 and .52 . For this reason, the authors concluded that the NNAT as well as the CPM could be used to predict academic achievement and would not present bias for students with language or other barriers to testing. 
Naglieri had previously conducted a similar study with Ronning that was published in 2000. Their hypothesis was based upon previous research by Naglieri in 1999 and made the argument that the measurement of general intelligence does not require both verbal and nonverbal content. Furthermore, nonverbal aptitude testing alone could accurately predict not only general aptitude or intelligence, but also academic achievement in specific content areas. To test this hypothesis, 22,600 students were given the NNAT as well as the Stanford Achievement Test 9 (SAT-9), a commonly used standardized test used in many K-12 schools nationwide. The sample included students in Grades $\mathrm{K}-12$ and the results from the NNAT and the Total Reading component of the SAT-9 were compared. In this study, they found that their hypothesis was supported. Looking across the grade levels, the lowest correlation found was .49 at grade 2 up to .61 in grade 11. The overall average correlation was .56 .

This result was similar to other correlations referenced within the discussion that referred back to similar studies of the Wechsler Intelligence Scale, the Differential Ability Scale, the Woodcock-Johnson Tests of Cognitive Abilities, and the K-ABC. Each of these additional tests includes components that include verbal and quantitative batteries, as well as nonverbal components. The researchers then concluded that general aptitude testing need not include both verbal and nonverbal components because the NNAT showed similar results to the test that included both verbal and nonverbal components. A limitation of the review, however, was none of these correlational studies were compared to the Naglieri and Ronning (2000) study that included the same indicator of academic achievement. Additionally, the sample used by this study included mostly suburban and rural students, leaving urban students under-represented in this study. 
Bliss (2008) completed her dissertation investigating the concurrent and predictive validity of the Universal Nonverbal Intelligence Test-Group Ability Test (UNIT-GAT). This new test included a nonverbal component. It was still being normreferenced as the dissertation was finished. This dissertation used a number of tests for comparison purposes with the UNIT-GAT; the nonverbal aptitude test selected was the NNAT. The purpose of the UNIT-GAT was to not only test reasoning as many tests do, but also memory. Because the NNAT does not test memory, the results of the UNITGAT that were utilized when comparing itself to the NNAT did not include the memory portion of the test. The UNIT-GAT portion of the test results is known as the Nonsymbolic Scale. This portion of the UNIT-GAT had a positive correlation of .50 ( $p<$ .01 ) with the NNAT. However, the sample included students only in the $2^{\text {nd }}, 5^{\text {th }}$, and $9^{\text {th }}$ grades. Across all three-grade levels, there was a decrease in correlational strength. The author attributed this to error in the UNIT-GAT scales as they had also showed a decrease in internal validity, through the grade levels. This study illustrated that a component of a newer test designed to test a similar construct as the NNAT did show a strong correlation with the NNAT.

Studies of exclusively nonverbal tests being used to predict academic achievement lead the researcher to the NNAT. Unfortunately, there is limited research available in this area that can be utilized by an admissions department. The little research that is available does not focus on the secondary level in education, nor does it identify a correlation between the results of the testing and academic achievement as identified by GPA. 
Reducing racial and economic bias. Utilizing a test for its intended purpose is important as its design establishes the expected range of knowledge, skills, and abilities the test taker is expected to be familiar with (Kokhan, 2013). A comprehensive assessment that is designed to gauge gaps in one's learning for example could be an extremely valuable tool for educators in identifying them for the sake of addressing them in a student's education. Conversely, a comprehensive test that expects a common culture or background in exposure in education and language used to evaluate a school system would present a biased interpretation of a student's potential if the same test were used for placement into rigorous schools or programs within a school. If a test is repurposed, it is being misused without testing to ensure it is valid and reliable in its new purpose (Kokhan, 2013).

Reducing this bias is important to provide minority students access to rigorous schools and programs (Naglieri \& Ford, 2003). While minority students may come to a school with a different culture and different background knowledge, it does not mean they do not possess the ability to learn and succeed. When investigating nonverbal aptitude tests, the most commonly mentioned tests are the NNAT, the Wechsler Battery, and the $\operatorname{CogAT}$. The biggest difference between the three is that the NNAT is the only one of the three that is comprised exclusively of nonverbal components, whereas the $\operatorname{Cog} \mathrm{AT}$ and the Wechsler battery both have verbal and quantitative elements that help to comprise the test (Lakin, 2012; Wechsler, 2003).

Giessman et al. (2013) analyzed data from one particular mid-Western school district to gauge the NNAT2 and the Cognitive Ability Test 6 (CogAT6) to predict the score a student would achieve on the WISC-IV, or one of the Wechsler batteries for 
assessing student intelligence. In doing so, they found that the NNAT2 and the CogAT6 produced similar results in predicting the WISC-IV scores. When looking at subgroups, however, the NNAT2 identified more ELL students and Hispanic students as having a high aptitude. Blacks, however, seemed to be disproportionately identified as having a low aptitude. Both tests also demonstrated a positive bias towards Asian students. As students progressed from kindergarten to second grade, the NNAT2 seemed to also have less varied results - fewer students were identified as having a high aptitude. The study only included these grade levels, however, and did not look at secondary education. Additionally, the CogAT6 data only came from second graders, therefore potentially distorting results as the entire sample was from an older grade level than the NNAT2 takers. Due to the differences in samples, it would be inappropriate to draw conclusions from this data.

In 2012, Lakin and Lai tested the CogAT with students in two schools with large Hispanic populations. The students in this study came from the third and fourth grades. They had students who were both ELL students as well as non-ELL students. When looking at the results of the two groups, the researchers came to some interesting conclusions. One, each portion of the CogAT test, to include the verbal, quantitative, and nonverbal portions, was all analyzed in regards to the two samples and the verbal and quantitative portions of the test were found to be the least generalizable. Additionally, the ELL students' results on the quantitative and verbal portions of the test presented range restrictions meaning the tests were statistically seen as less precise. While this multidimensional test is seen as better being able to predict academic success in students than a nonverbal test alone by some (Lohman, 2005), Lakin and Lai (2012) recognize 
there is a need for further research into nonverbal testing as well as multi-dimensional testing to more accurately gauge student ability. Additionally, they see further questions need to be addressed in regards to a single test is being used for placement into gifted programs.

In an additional study performed by Lakin in 2012, she looks at the complete battery of the $\operatorname{Cog} \mathrm{AT}$ to include its nonverbal, verbal, and quantitative sections administered to $3^{\text {rd }}$ and $4^{\text {th }}$ grade students to identify the differing components' correlation with results on a state standardized test, namely the Arizona Instrument to Measure Standards Dual Purpose Assessment. As she introduces her work, she argues that an ability test should measure not only innate ability, but also well-practiced reasoning skills. Because of this, a test that shows lower scores for ELL students is not biased, but rather identifies distinct information. She then argues that the ability tests identify important information for educators in working with ELL students to close an achievement gap. In conclusion, she argues against the exclusive use of nonverbal tests if the students have some background in English as the tests may reveal valuable distinct information. At the same time, she does acknowledge the verbal reasoning tests' limitation in their psychometric qualities, reliability and validity, in relation to ELL students.

Lakin (2012) identifies the benefit of a multivariate test over a one-dimensional test. Namely, the identification of shortcomings a student may possess that can help adjust the education a student receives to better support them. The issue with this is that if that same test is a main component of a screening process to enter a rigorous school or program, it could be biased against students with differing backgrounds. Knowledge that 
multidimensional tests become less generalizable when dealing with ELL populations helps to highlight the inherent bias that these tests can possess when working with diverse students.

The Wechsler battery of testing has multiple components that include nonverbal, verbal, and quantitative testing, similar to the CogAT. It is commonly used in testing for admissions processes to reduce bias due to its nonverbal component. Due to its reliance on both verbal and quantitative components, Verney and his fellow researchers established a psychophysiological test to measure students' intelligence through visual stimulus and response. When the results of this testing were correlated with the Wechsler, apparent differences presented themselves when looking at differing samples. It was found that students' psychophysiologically base measured response scores accounted for approximately $17 \%$ of the variance in their Wechsler scores. When analyzing the results of Caucasian American students, the results were found to be significant. When looking at the scores for Mexican Americans, the results were not significant. The finding of differential validity was seen as presenting test bias in the Wechsler due to differences in the students' backgrounds (Verney et al., 2005).

Naglieri (2006), being an advocate of testing that reduces negative bias toward minority students to promote more diversity in advanced programs, encouraged the use of nonverbal testing. His recommendation is based upon some of his own prior work. In 2003, Naglieri and Ford conducted research with the NNAT to identify its ability to promote minority children access to gifted education due to their underrepresentation in gifted programs. When looking at the average score on tests utilized to screen students into gifted programs, they looked at the difference in the means of the results across 
racial lines between Whites, Blacks, and Hispanic students. They found the one form of the Wechsler, the WISC-III, to have an average difference of 11.0 between different races. On the Stanford-Binet-IV, there was a difference of 8.1. With the WoodcockJohnson Tests of Cognitive Ability, there was a difference of 11.7. The large discrepancy in test results indicates that fewer minority students would be identified for inclusion in gifted programs than their representation in the general population. In this study, 20,270 students were given the NNAT. Demographically, the students showed diversity that in many ways reflected the United States population to include racial, socioeconomic status (SES), urbanicity, and regional dwelling. In comparing the means across racial lines, the averages ranged from 96.1 to 99.3 . If a cut-off of the $95^{\text {th }}$ percentile were used to place students into gifted programs, then White, Black, and Hispanic students would have approximately equal representation in the gifted programs. The representation would be $5.6 \%, 5.1 \%$ and $4.4 \%$, respectively (Naglieri \& Ford, 2003). It may then be inferred that the NNAT does reduce bias based on racial and cultural differences with the sample used in this study. This particular study only reviewed the difference of means in students, however, and did not attempt to predict academic achievement, or identify the number of students in each racial group that would be identified for gifted programs at a given cutoff rate.

In Lohman's (2005) review of Naglieri and Ronning (2000), he criticizes their findings for a couple of reasons. First, he thought a limitation of the study was that merely $5.6 \%$ of the students were derived from urban school districts, yet a much larger portion of the population in the United States lives in urban areas. Secondly, while there were a large number of Black and Hispanic students, they were from significantly higher 
SES statuses than in the general population. He argues that this would skew the results as SES also plays a significant role in predicting achievement. He considers this approach to be biased because the researchers already had the data when producing the new statistical models to obtain their findings. For these reasons, he expresses his doubt in the results that they released. He then goes on to argue that a test such as the CogAT does a better job of identifying students who should be in gifted programs because it includes language and when combined with its nonverbal component, better predicts academic success. He argues that SES is what equals the playing field in Naglieri's (2006) findings rather than the test itself.

In reviewing this critique of Naglieri's work, the issues that he discusses may skew the results and potentially present bias. On the other hand, one must consider that a researcher will not always be able to obtain a large random sample as preferred, but may need to depend on convenience samples in some instances. The author may also be questioned fairly as to why he pushes the CogAT in review of Naglieri's research, knowing that Lohman is one of its creators.

In reply to Lohman (2005), Naglieri and Ford (2005) published a response in which they stress five main points that look at both the NNAT as well as the CogAT. The first point made is the reiteration of the need to identify students of all cultures to be included in gifted programs. The NNAT attempts to do this by down-playing potential bias in the test. Point number two: while the CogAT may be seen as a better predictor in some cases due to its inclusion of language and mathematical portions, it still places students from a lower SES as well as minority students at a disadvantage. They may not have had the same introduction to these types of problems in the past or if they are not 
native English speakers, the verbal component will not be accurately gauging their intelligence. Their third point is that the CogAT has a stronger correlation to the Iowa Basic Skills Test than the NNAT because they both test using similar prompts, further replicating potential bias as found in the Iowa Basic Skills Test to students from differing backgrounds. Fourth, the CogAT rewards previously academically gifted students rather than identifying intelligent students who have the ability to learn. Their last point is an open invitation to provide evidence that the NNAT creates a large discrepancy in identified students of differing races in application, for gifted programs.

Carman and Taylor (2010) looked at the NNAT to identify any disparity, if it existed, between students of differing SES, as well as race. They found that students of low SES scored significantly lower than those of a higher SES, after controlling for ethnic differences. This finding was in fact, in line with a previous study done by Drake(2006), who found that students of different ethnicities were identified at substantially different rates as being "gifted." When looking at students of low SES, she found the differences to increase. However, both of these studies focused on students at a much younger age, kindergarten and kindergarten through 6 , whereas the present study is looking at students in secondary education. As previously indicated by the Naglieri and Ford research done in 2003, the NNAT did not eliminate differences between races, but reduced them.

One of the goals of the NNAT is to reduce bias (Technical Information, 2011). This does not mean that it will be completely eliminated, although it would be highly desirable. This study may have also skewed the results for Caucasians, the majority, by incorporating Asian students into the results. Carman and Taylor (2010), as well as 
Giessman et al. (2013) identified this group as demonstrating a positive bias in testing. This would have inflated the test results for the majority, Whites, and created larger differences amongst the means between the races identified. This leads one to desire further information to better understand the results.

Lewis (2001) tells us that language is not needed in assessing students to identify gifted learners. She argues that utilizing language encourages exclusionary practices with students of diverse backgrounds who speak different languages than the majority. In her research, she mentions that research with nonverbal testing to identify gifted students is relatively sparse.

She identified two previous studies that had been done utilizing the NNAT, Raven's Matrices, and the Culture Fair Intelligence Test (CFIT), a test that is used to yield an IQ score. All three are exclusively nonverbal tests. She wanted to identify how well each of the tests did in identifying students for gifted programs amongst Whites, Blacks, and Hispanics. The two studies included 459 students in grades 3 through 8. One of the studies was done in the South and one in the Midwest. They both included impoverished populations, one with a large number of Blacks and the other with a large number of Hispanics. In this published paper, both samples were briefly discussed. The data of one of the studies that was presented was broken down by race and each particular test. The grade levels of the students were ignored. If a student scored above the $80^{\text {th }}$ percentile, they would be recommended for gifted programs.

This led Lewis (2001) to ask the question, "Which test is best?" She goes on to tell the reader that depending upon the study, the most efficient was either the CFIT or Raven's and stated that their identification rates were well above 50\% with their sample. 
This would mean that over half of those tested were seen as being at or above the $80^{\text {th }}$ percentile of each of the test's norm referenced groups. This is the criterion she used to argue that the NNAT was not as useful. She then identified each test that seemed to be better for each particular race: the CFIT for Hispanics, and Raven's for both Black and White students. From the data presented, it appears that the tests identified proportionally similar numbers of students across racial lines; however, this is difficult to ascertain as it is not clearly made known in the data shared. Unfortunately, there is no follow-up to know whether the students identified actually began to study in an honors program or to track their academic achievement.

Lewis (2001) encourages yearly screening of all students using multiple tests to identify as many students for gifted placement as possible and to remove teacher judgment from the decision-making process. Her study seemingly does little to identify whether it identifies students fairly across differing demographic lines or helps to predict academic success. This research does not look at the racial means on the tests to identify bias, but merely asks which test places the most students into a "gifted" category and based upon that information, speculates as to which test benefits each race the most.

Saint Paul, Minnesota Public Schools' Office of Research and Development published a Gifted Services Identification Report in 2006 (Drake). It identified their method in which kindergarten and second grade students were referred to gifted services in kindergarten and in second grade. Following the example of other school districts, they incorporated the NNAT into their screening process and students who were at or above the $92^{\text {nd }}$ percentile on the NAI were automatically referred. Those between the $85^{\text {th }}$ and $92^{\text {nd }}$ percentile would be reviewed by means of a portfolio for consideration to 
be referred to gifted services. They published data from early in 2006 concerning the testing that took place.

When looking at the data, they encouraged further review of the process, as their results did not show a testing process that was completely free of bias. The results were broken down by gender, ethnicity, home language, whether the students were ELL, as well as SES level as determined by whether a student had free or reduced lunches. In looking at gender, the number of students referred to gifted services was similar. Regarding ethnicity, gifted services were recommended for approximately $13 \%$ of American Indian, Asian American, and Hispanic American students, whereas only 6.5\% of African American and 30\% of Caucasians were recommended for gifted services. Only $11.5 \%$ of ELL students were referred as compared to $18 \%$ of non-ELL students were referred. Approximately $30 \%$ of students in the sample who received free or reduced lunches were referred for gifted services, and about $30 \%$ of the students who did not qualify for free or reduced lunches were referred as well. Approximately $20 \%$ of the students who spoke English in the home were referred, while about $10 \%$ of students who spoke either Hmong or Spanish in the home were referred for gifted services. These discrepancies do not necessarily portray a test that is without bias.

The authors encourage further review to see whether the numbers change significantly in the future or whether they remain consistent. The numbers presented in this study do encourage further research. In considering the research in this study, however, one sees that again, the grade levels reviewed by St. Paul were much younger than the students who are the focus of this research paper. 
Villarreal (2005) completed a dissertation looking at the reliability and validity of the Naglieri Nonverbal Aptitude Test with ELL Mexican American students.. He wanted to test some of the assumptions made by the publishers of the NNAT. The publishers of the NNAT published internal validity results to support its validity as well as made the statement that the test helps to remove bias based upon gender, SES, and race (Technical Information, 2011). The internal consistency identified by the sample in this dissertation with ELL Mexican American students did not differ from the results obtained by the publishers in that only the reliability coefficients for the NAI total score were published. Villarreal did identify discrepancies when looking at individual components of the test, but the NNAT only publishes the internal validity for the entire battery. Secondly, the author wanted to see if the performance of his sample would replicate the norm group of the test. If so, the assumption can be made that similar numbers of students from the norm based group as well as from the dissertations sample would be identified as having a high cognitive ability. In performance, there was no statistical difference. When looking at individual clusters of the NNAT and the results obtained by the norm group and the sample group of that dissertation, some differences were identified that may encourage further review, similar to identified discrepancies with internal validity. The overall internal consistency, however, and the performance levels achieved showed no statistically significant difference.

\section{Synthesis of Research Findings}

The existing literature surrounding the use of nonverbal tests to gauge academic aptitude, although limited, seemingly encourages their use. They would help schools to neutrally gauge students' aptitude across ethnic, linguistic, gender, economic, and 
cultural lines. Existing literature, however, seems contradictory when one test is pushed over another. The majority of the literature implies that nonverbal aptitude testing does predict aptitude. However, the question most commonly repeated is whether tests that also utilize verbal and quantitative components are better at predicting academic success. Proponents of strictly nonverbal tests argue against tests with verbal and quantitative components as they are seen as presenting bias.

The current literature does not lead to any definitive conclusions as the articles tend to speak against one another. The perceived bias in this regard due to the articles' authorship necessitates further testing. Additionally, when analyzing the samples of the studies, cross-sections of society do not seem to be well represented and often focus on elementary students rather than secondary students.

This study looks to gauge the predictability of academic achievement using a nonverbal aptitude test, namely the NNAT. The validity of nonverbal testing in the current literature, however, is often shown by correlations made with other aptitude or standardized tests, rather than academic achievement. This research is an extension of prior research then in two ways: (a) it is designed to test the link between nonverbal aptitude and academic achievement, and (b) it examines this hypothesized relationship with secondary students, rather than elementary-aged students.

\section{Chapter Summary}

Learning takes place in a student's life that is most often based upon that student's culture and language. The ability to think logically and find solutions to challenges is not generally limited by the language used solve the problem (Naglieri, 2006). If a problem is given to a student void of language, the student must still process the information given 
to find a solution. If a nonverbal test is able to provide evidence of reasoning skills without presenting problems that are tied to language that may be biased against students due to language and cultural issues, then the test may benefit schools in placing students appropriately into challenging programs. This does not mean they will not need support, but that with support, they can function at a high level until they are able to stand alone.

Bandura's (2002) SCT has posited that a learner can influence his environment as well as those around him. Conversely, the environment as well as those around the learner can influence the learner. A school that establishes high expectations and provides support to students to function at a high level regardless of background will promote success regardless of a student's background if they have the aptitude necessary within a given environment to be successful.

To gauge SCT theory then, further study needs to take place in which students' nonverbal aptitude scores are evaluated as to their ability to predict academic success in a school environment. This study sought to do so in a small secondary setting with a high percentage of Hispanic students who may be seen as disadvantaged in traditional aptitude testing done in English due to their backgrounds being dissimilar from the White majority. 


\section{CHAPTER III}

\section{METHOD}

\section{Introduction to Methodology}

In the previous chapter, a literature review was done to accomplish two goals.

First, it established the theoretical framework behind this study, Bandura's (1989, 2002)

Social Cognitive Theory (SCT) as well as defining the construct, nonverbal aptitude.

Bandura's SCT tells us that through a triadic relationship, a learner grows through interaction with his or her environment as well as through others. This learning process within a supportive and challenging school will encourage the student to do his or her best. This leads into the second goal of the literature review, to identify the need for a study of the nonverbal aptitude to gauge its predictability of academic success in a school that exhibits these characteristics. The following factors were identified for investigation: the predictability of academic success based on nonverbal aptitude while controlling for grade, gender, whether the student is a native English speaker, and ESL level; the mean of nonverbal aptitude scores for males versus females; and the mean of nonverbal aptitude scores for native English speakers as opposed to non-native English speakers.

This chapter will deal with the research methodology used in this study. To test the hypotheses of this study, ex post facto methodology was employed at a private secondary school. Data on new students were collected for a 3-year period (2013-2014, 2014-2015, and 2015-2016) to increase the sample size. Because the study was performed at one site, all new students were utilized in the study, and therefore the sample was in essence a convenience sample. Because the school already collects the data needed, an IRB exemption was applied for and granted. 


\section{Purpose of the Study}

The purpose of this study was two-fold. First, it sought to examine to relationship between nonverbal aptitude as measured by a nonverbal assessment and academic achievement obtained by secondary students in a private school. The student population is one that is primarily Hispanic and comes from numerous countries around the globe. A hypothesized positive relationship would show a nonverbal aptitude assessment as being able to predict academic achievement accounting for unique variance when controlling for grade level, gender, primary language spoken, and ESL level, as measured by WIDA, as well as the multi-collinearity between these controlled variables. By extension, it would show that it would facilitate the effectiveness of the admissions process in a similar school. Secondly, it sought to determine whether there was gender or language bias in nonverbal aptitude with this sample of secondary school students (grades 6-12).

\section{Research Questions}

Three overarching research questions shaped this study: (1) Is there a relationship between student's nonverbal academic ability and academic achievement while controlling for gender, grade level, whether the student is a native English speaker, and ESL level? (2) Is there a significant difference in the nonverbal academic achievement mean scores of the applicants due to gender? and (3) Is there a significant difference in the mean for academic achievement scores of native English speaker versus the nonnative English speakers that test? 


\section{Research Hypotheses}

1. Nonverbal aptitude as measured by the NAI score, the independent variable, accounts for a significant amount of unique variance in predicting academic achievement as measured by second semester GPA, the dependent variable, when controlling for grade level, gender, primary language spoken, and ESL level as measured by WIDA at the secondary grade levels (6-12).

2. There is a significant difference in nonverbal aptitude mean scores as measured by the NAI score at the secondary grade levels (6-12) by or between the genders.

3. There is a significant difference in nonverbal aptitude mean scores as measured by the NAI score of non-native English speakers as compared to native speakers at the secondary grade levels (6-12).

\section{Design of the Study}

The framework of this study is modeled partly after similar studies of nonverbal aptitude tests performed by Naglieri and Ronning (2000) in testing the validity of the NNAT. The researchers conducted ex post facto correlational studies that showed the test's validity by demonstrating a positive correlation with SAT-9 scores, as well as the SAT-9's differing sub-categories scores, such as: total reading, vocabulary, and reading comprehension.

In this study, the researcher utilized an ex post facto correlational design that identified the relationship between secondary students' nonverbal aptitude measured at application time and the subsequent GPA earned during their second semester at the school. The higher the positive correlation that exists, the better nonverbal aptitude is able to predict academic achievement as measured by GPA. 
Knowing that the environment has a relationship with the student's regulatory processes and actions, the student's grades were not evaluated until the second semester. The assumption is made that in this time, new students have become acclimated to the school, know its expectations, and have built relationships. At this point, the student should be comfortable in his or her surroundings and have established friendships as well as identified role models within the school. Seeing positive examples and receiving the support of fellow peers and faculty at a demanding yet supportive school may well shape the student's perception of himself or herself to include traits of motivation, as well as self-efficacy. This is important because research implies that student motivation is just as powerful in predicting student achievement as student aptitude (Cote \& Levine, 2000). Self-efficacy, in fact, has been shown to be reflected in the amount of time one is willing to spend on a particular task (Vera, Le Blanc, Taris, \& Salanova, 2014). This only makes sense when one understands that the belief in success is a motivational force.

Additionally, Bandura (1994) has identified the importance of social experience within social settings to strengthen these types of motivational factors. Sivan (1986) points out that within a classroom environment, there is a joint activity between the student and the social setting that has an impact on both cognitive and affective engagement as well as on motivational behaviors. The environment is thus seen as a powerful force behind student achievement when coupled with student aptitude.

In a predictive study, implications are made pertaining to data collection, analysis, as well as interpretation. A dependent variable must be identified, as well as variables that may directly be related to it and in fact predict its outcome, known as independent variables. This type of study does not imply causation, but predictability (Reio, 2016). 
Additionally, variables that may skew the predictability of the independent variables must be controlled for statistically (Kaya, 2015).

In addition to testing the association between nonverbal aptitude and GPA, the results of select groups were compared to identify potential statistically significant differences between them. This included two comparisons: a comparison of the mean scores of males and females, as well as a comparison of the mean scores of native and non-native English speakers.

This study is driven partly by a desire to test Naglieri's (2006) notion that nonverbal intelligence testing can identify secondary students with a high aptitude in an unbiased manner to place them successfully into challenging courses and programs. Prior research has not looked to identify a correlation between secondary students' nonverbal aptitude and their earned GPAs. This research took prior theoretical research in which nonverbal testing was correlated with other tests and moved it into applied research that may be meaningful to educational leaders and admissions personnel who seek to identify students of diverse backgrounds in an unbiased manner.

\section{Research Variables}

The dependent variable in this study is academic achievement. Academic achievement was measured by taking each student's overall GPA during the second semester at the school. This action was taken to give the student time to acclimate him or herself to the school and its expectations, as well as its culture and student body keeping in mind Bandura's $(1989,2002)$ SCT that shows a student and his or her learning is impacted by social interaction and as a reaction to the stimulus provided in those interactions. Academic achievement is important in this study because it is identified as 
the key indicator of success in education for a student. The grade or GPA earned may reflect a number of things to include motivation, determination, hard work, prior education, parental involvement, and aptitude; along with numerous other items (Allan, 2005). Because a grade reflects more than just aptitude, one cannot expect a perfect correlation or anything near it. This is why the study seeks to discover the predictability of GPA based on nonverbal aptitude test scores in a diverse private secondary school setting.

The independent variables include nonverbal aptitude, gender, grade level, whether the student is a native English speaker, and ESL level.

One component of this study is to see whether the nonverbal aptitude testing in this study has reduced bias due to gender. By identifying this variable, the results of the test can be statistically tested to see whether it does remove the bias.

Another component of this study is to see whether the nonverbal aptitude testing in this study has reduced bias based on whether a student is a native English speaker. The native language a person speaks in and of itself does not dictate a student's aptitude. By identifying this attribute in prospective students, this study can determine whether there is bias against non-native English speakers, which schools may be working to avoid.

The ESL level of a student lets the school know how proficient a student is in the English language. The differing levels are defined in the Access program developed by WIDA. WIDA is an ELL assessment program developed by numerous states for gauging ELL student proficiency levels. ESL levels range from one, "entering"; to six, "reaching," according to WIDA. These values may be seen as being representative of a 
student's ability in the use and comprehension of language ranging from a beginner to native level user of the English language. The values, while continuous, are not seen as interval data as each decimal represents a proportion within a proficiency level and not seen as equivalent to a decimal place within another proficiency level (ACCESS for ELLS, 2011).

It is important to control for this variable so that the English level of non-native English speakers is acknowledged, as acquiring the English language is a process. There is a difference in English ability levels, and by accounting for the potential effect of that difference in the regression equation, the more accurate the results in interpretation.

\section{Sample and Setting}

At the preliminary stages of this research, numerous private schools were contacted in South Florida, as well as the Association of Independent Schools of Florida (AISF), an independent accreditation body, to identify multiple schools for inclusion in this study. Only one school was willing to help in this research as they had implemented nonverbal aptitude testing four years ago as part of their admissions process. This school's decision was based upon the materials distributed by Pearson in the school's search for an admissions test their applicants would be able to take despite potential language or cultural barriers. The school used this test in conjunction with an interview process as well as looking at prior grades to better determine whether accepted students would have the potential to be successful in the school. Additionally, testing provided by WIDA was used to identify ESL level. Due to the number of applicants it receives each year and the school's growth, an $n$ size of approximately 200 is achieved by pulling three years' worth of data. This goes back to when the school began using these tests. 
The school had reviewed the SSAT for use in their school in conjunction with the SSATB and had done a trial of it with its students. Due to the background of the student body, it was found to be dysfunctional as it depended on an extensive knowledge of the English language as well as American history and culture.

The AISF has asked the researcher to provide a workshop for administrators on the topic, provided that the first hypothesis holds true upon completion of the study.

Due to the limited response, the study was done at one small private school in South Florida that currently has 850 students. It is a Christian school and its teachers are trained and certified as teachers within the church body it is affiliated with, as well as in either Minnesota or Wisconsin, depending upon the college that they attended. Upon moving to Florida, the teachers apply for Florida certification. The church body either operates or is affiliated with one college in each of these states. The school is utilized as a ministry tool of the church. In its mission, it identifies the need for excellence in education. The school houses Pre-Kindergarten 3 through the $12^{\text {th }}$ grade. It is located in an affluent area and works to serve the local community in which it operates. For this reason, most of its students live locally and form a diverse student body. The school is considered a majority-minority school as the demographics show that there are more Hispanics, a minority in the United States, than students of the majority in the United States, White non-Hispanics (Grinberg, 2011). Additionally, the high school hosts numerous international students, and this is expected to grow as they expand their international program. For this study, only secondary grade levels were examined, that is, grades 6 through 12. The school's current enrollment demographics are as follows: $82 \%$ Hispanic, $10 \%$ White non-Hispanic, 3\% unclassified, and $2 \%$ other. 


\section{Sample Size}

The researcher's main concern with being limited to one school was finding a way to optimize the sample size. Green (1991) states that a correlational analysis requires a minimum of five participants for each variable that is included in the study, with up to 50 being recommended. Five variables have been identified for this study requiring a minimum of 25 participants, but the researcher aimed for a minimum of 100 participants to increase statistical power (Green, 1991).

The sample for this study includes new students to the school in grades 6-12 over a span of three academic years from 2013 to 2016. In 2013-14, there were 53 new students to the school in the specified grades, 2014-15 had 77 new applicants, and there were 86 for the 2015-16 school year. With the compilation of all three years' worth of data, the combined sample size was 203 students (once excluded students based upon the exclusion criteria were removed). Thirteen students were excluded from the study for not completing a second semester at the school.

Using an estimate of statistical power based upon Cohen (1988), where the effect size is medium and the alpha level is set at .05 with a sample size of approximately 200 , we find a power over 90 .

\section{Inclusion Criteria}

All students who were accepted to the secondary school, grades 6 through 12 , from 2013 through 2015 were included in the sample. It included a convenience sample of all accepted applicants into the school in these grade levels who were tested prior to acceptance and were present for two consecutive semesters. 


\section{Exclusion Criteria}

Out of the convenience sample, the only students who were excluded when the data were collected were those who started mid-year during the 2015-16 school year. The second semester GPA would not reflect their second semester at the school, and it would potentially skew the results as they would not be given time to adapt to the school (Bandura, 1989, 2002). Additionally, students who did not finish their second semester were excluded since the required data for those students would not exist. Finally, students who were not accepted into the school are not included in the study as they did not earn grades in the school that were able to be recorded, nor would it be known if their grades from another school would present a similar grading philosophy or scale.

\section{Ethical Considerations}

The researcher applied to both FIU's IRB for approval as well as to the administration of the school where the study was done to conduct the research. Upon receiving approval from the IRB and the school, the researcher began conducting the study. The admissions process of the school already utilizes a measure of nonverbal aptitude and therefore minimal additional risk or benefit was presented to the students, as the school's admission process was already complete. This study only looked at existing information that was already collected by the school and/or provided by the parents to the school at the time of application. Because of this, the data needed already exists and the researcher was not required to conduct further testing. Following IRB protocol, the records were coded to hide names and they were kept confidential, as the computer that the information was stored on was also password protected. The test results were kept in electronic form only on the password-protected computer without names or other 
identifying information other than what was required. The spreadsheet of information only contained a number assigned in the place of each student's name not linked to the name for reference, grade level, gender, whether the student is a native English speaker, and ESL level.

\section{Research Instrument}

The NNAT, currently in its second edition, was developed to measure general ability with nonverbal measurements in the form of progressive matrices, or picture puzzles that challenge one's logic with intricate patterns. This was done to promote fairness regardless of gender, race, and ethnicity (Directions for Administration, , 2011). It is a standardized test that can be administered in a short amount of time in a group setting by most educational personnel. It avoids the usage of language within the test itself to remove language bias. Through the administration of the test, all information needed to complete the questions is presented within the questions themselves nonverbally. This attempts to remove all forms of bias as there is no language in the test or previous conceptual or mathematical principles that have to be mastered to perform well. The test itself is given over 30 minutes and includes 48 questions that are based upon progressive matrices utilizing three colors: white, yellow, and blue. The matrices present challenges to students that incorporate reasoning, memory, pattern recognition, and speed of processing (Technical Information, 2011). The problems are very easy at the outset and progressively get harder. Moreover, there are different test forms for differing grade levels to avoid any ceilings that might be reached by high achieving students. At the end of the test, the students are given an NAI score that is based upon a 
mean score of 100, and students who score over 100 are seen as having an above average intelligence (Technical Information, 2011).

The NNAT2 utilizes its own scoring mechanism, the NAI score, to quantify a student's Academic Aptitude. Using inferential norming and conversions to standard scores, the mean score on the NNAT2 or the NAI score is 100 with a standard deviation of 16 and a range of 40-160 (Technical Information, 2011).

\section{Reliability}

Internal consistency reliability was estimated by the split-half reliability method. The 48 questions in each level of test were split in half based upon the item difficulty estimated by the IRT (Technical Information, 2011). The split-half reliability coefficient, utilizing the Spearman-Brown formula, resulted in an overall .90 reliability coefficient. Internal consistency was obtained using Kuder-Richardson Formula 20, resulting in a .88 reliability correlation (Technical Information, 2011). Additionally, the mean across differing racial and ethnic groups, African American, Hispanic, and White, only had a range of 4.2 points on the NAI. When controlling for SES, school type, urbanicity, region, age, and gender, the range only differed by .5 points on the NAI, demonstrating fairness across ethnic lines (Technical Information, 2011).

\section{Validity}

When analyzing the correlation between the Wechsler Nonverbal Scale of Ability's matrices, a subtest of the Wechsler, and the NNAT2, a .73 correlation is shown, providing for concurrent validity of the NNAT2 (Technical Information, 2011). Evidence for validity is restricted to correlations with the Wechsler Nonverbal Scale of Ability matrices. In a dissertation analyzing the reliability of the Naglieri Nonverbal 
Aptitude Test, Villarreal (2005) found that the Cronbach's alpha correlations did yield evidence supporting internal consistency with third and fourth Grade ELL Mexican American students on the NAI. Manos (2011) tested the construct validity of the NNAT2 with a sample of 52 Black and 367 White students. He found that the average item performance for each group was similar. The rank order difficulty with Spearman's rho $=.93(p<.000001)$. The mean point-biserial correlations were .35 and .39 respectively for Black and White students, demonstrating the construct validity for each group.

The most important aspect of validity for an aptitude test is its ability to predict academic achievement (Naglieri \& Ronning, 2000). The authors of the test have attempted to demonstrate this point by showing validity with other tools designed to measure general ability. This current investigation was undertaken to verify this point by performing a correlational study of the NAI score results and academic achievement obtained by the sample.

\section{Data Collection Procedures}

With IRB guidance, all new students were identified from the appropriate grades, grades 6 through 12 ,during the academic years from 2013-2016. The researcher pulled each of the identified student's files and recorded the necessary information for this study, to include grade level entering, gender, ESL level, whether their primary language is English,, nonverbal aptitude score (NAI), and their GPA after their second semester at the school. To assure confidentiality, each person's name was replaced first by a numerical number representing the number they represented in the convenience sample. The information was not attached to a name but rather a number, and was analyzed using SPSS as outlined in the analysis portion of this report. 


\section{Data Analysis}

All analyses were carried out using SPSS Version 18.0. The significance level was set to $a<0.05$; a one-tailed test was applied to test for positive significant correlation as anticipated by the hypothesis.

Demographics and base line grades are described in chapter 4. The descriptions are based on mean, standard deviation, median, minimum and maximum for continuous variables; GPA, NAI, and ESL level; and frequency and percent for nominal; Gender and whether a student is a native English speaker, and the ordinal variable, Grade Level.

\section{Testing the Hypotheses}

1. Nonverbal aptitude as measured by the NAI score, the independent variable, accounts for a significant amount of unique variance in predicting academic achievement as measured by the second semester GPA, the dependent variable, when controlling for grade level, gender, primary language spoken, and ESL level as measured by WIDA at the secondary grade levels (6-12).

A hierarchical regression analysis was conducted using achievement as the dependent variable, and all other above mentioned variables as independent variables. The hierarchical regression method is the most useful for testing theoretical models that include numerous independent variables, introducing each variable one at a time in descending order of their ability to predict the independent variable while controlling for the remaining independent variables (Cohen, 1988). Predictors are entered cumulatively in a pre-specified order based upon the logic of the study to determine the R-squared and partial regression coefficient of each variable at each step and to identify the change in variance that 
is predicted by adding each variable to the model one at a time (Cohen \& Cohen, 1983). The dependent and independent variables in this hypothesis are continuous variables that are included in parametric data. Due to this fact, Pearson's correlation will be used to evaluate the correlation. The null hypothesis was the regression coefficient of the NAI score equals zero, provided a well-fitted regression.

To control for Type I error, or finding a false positive, the Bonferroni method was utilized. The Bonferroni method utilizes the alpha level, in this case .05, and divides it by the number of tests for significance, which in this case is five. This leaves us with an adjusted alpha level of .01 and $p$ is the calculated value and has to be less than or equal to the adjusted alpha of .01 (Newman, Fraas, \& Laux, 2000).

2. There is a significant difference in nonverbal aptitude mean scores as measured by the NAI score between the genders. Hypothesis two was tested using a $t$-test. The null hypothesis was that the mean NAI score for males was not significantly different than the mean nonverbal academic ability score for females as measured by the NAI score.

3. There is a significant difference in nonverbal aptitude mean scores as measured by the NAI score of non-native English speakers as compared to native speakers.

Hypothesis three was tested using a $t$-test. The null hypothesis was that the mean NAI scores for English as primary language students was not 
significantly different than the mean nonverbal academic ability scores for students with other than English as their primary languages.

\section{Chapter Summary}

Chapter 3 reviewed the methods of this study and how they serve to meet the purpose of this study. The research questions and hypotheses were revisited to understand how the design of the study would seek to answer the research questions while testing the hypotheses. Each variable included in the research was reviewed as to its theoretical and empirical importance. The sample utilized was reviewed to better understand the reliability and external validity that would be achieved in its use. Ethical considerations were discussed. The NNAT2 was also reviewed to better understand the instrumentation that would be used to gauge the nonverbal aptitude of students. The data collection method was reviewed as well as how this information was analyzed to obtain the data necessary to answer the research questions given the three hypotheses of this study. 


\section{CHAPTER IV}

\section{RESULTS}

This chapter presents the findings of the study. It is organized into three main sections. The first section presents demographic and background information of the sample itself. The second section examines the hypotheses. Finally, a brief summary of the chapter is presented.

\section{Description of the Sample}

A brief review of the sample is presented here to better understand the presented results. The study was conducted at one small private religious school in South Florida that currently has 850 students. The majority of the students at the school, which serves students from Pre-Kindergarten 3 to Grade 12, are from the local community. The community is an affluent area that is diverse demographically. The school replicates this diversity within its student body. Additionally, the high school hosts numerous international students through its international program. For this study, only secondary grade levels will be examined, that is, grades 6 through 12. The school's current enrollment demographics are as follows: $82 \%$ Hispanic, 10\% White non-Hispanic, 3\% unclassified, and $2 \%$ other.

The sample included 203 students who were accepted into the school in grades 612 over a span of three academic years from 2013 to 2016 after removing 13 students who were excluded based upon the exclusion criteria.

Demographics and descriptive statistics of continuous variables for the sample will now be presented in Table 1 (Frequency Table of Demographic Variables) and Table 2 (Descriptive Statistics of Continuous Score Variables) and then be described in detail. 
The description will be based on frequency and percent for nominal variable; gender and whether a student is a native English speaker, and the ordinal variable, grade level. Continuous variable descriptions will be based on minimum and maximum, mean, and standard deviation for continuous variables; $2^{\text {nd }}$ semester GPA, ESL level, and NAI score.

Table 1

Frequency Table of Demographic Variables

\begin{tabular}{|c|c|c|c|}
\hline Category & Variable & $f$ & Percent \\
\hline \multirow[t]{3}{*}{ Sex } & Female & 98 & 48.3 \\
\hline & Male & 105 & 51.7 \\
\hline & Total & 203 & 100.0 \\
\hline \multirow[t]{8}{*}{ Grade Entering } & 6 & 38 & 18.7 \\
\hline & 7 & 43 & 21.2 \\
\hline & 8 & 51 & 25.1 \\
\hline & 9 & 38 & 18.7 \\
\hline & 10 & 16 & 7.9 \\
\hline & 11 & 12 & 5.9 \\
\hline & 12 & 5 & 2.5 \\
\hline & Total & 203 & 100.0 \\
\hline \multirow[t]{3}{*}{ Native English Speaker } & Non-Native & 141 & 69.5 \\
\hline & Native & 62 & 30.5 \\
\hline & Total & 203 & 100.0 \\
\hline
\end{tabular}


Gender. Out of the sample of 203 students, 105 (51.7\%) students were males, and98 $(48.3 \%)$ of the students were female.

Grade entering. The frequency analysis of the grade level of accepted student entered indicated that $18.7 \%(n=38)$ of the students entered Grade $6.21 .2 \%(n=43)$ of the students entered Grade 7. $25.1 \%(n=51)$ of the students entered Grade $8.18 .7 \%(n$ $=38)$ of the students entered Grade $9.7 .9 \%(n=16)$ of the students entered Grade 10 . $5.9 \%(n=12)$ of the students entered Grade 11 and $2.5 \%(n=5)$ of the students entered Grade 12.

Native English Speaking. Out of the sample of 203 students, 62 of the students were native English speakers. 141 of the students were non-native speakers who were of differing English proficiency as indicated by their ESL level as measured by the WIDA measurement test.

Table 2

Descriptive Statistics of Continuous Score Variables

\begin{tabular}{lcccc}
\hline Category & Minimum & Maximum & Mean & Std. Deviation \\
\hline $2^{\text {nd }}$ Semester GPA & 1.231 & 4.000 & 3.067 & 0.57 \\
& 1.0 & 6.0 & 4.38 & 1.61 \\
ESL Level & 11.0 & 155 & 108.41 & 15.48 \\
NAI Score & & & & \\
& & & & \\
\end{tabular}

Note: $N$ Size $=203$ 
$2^{\text {nd }}$ Semester GPA. Out of the sample of 203 students, the minimum $2^{\text {nd }}$ semester GPA was 1.231, or a letter grade just above a "D" on a 4.0 scale. The highest GPA was a 4.0, or a straight "A" average on a 4.0 scale. The mean was a 3.067 , or approximately a "B" on a 4.0 scale.

ESL Level. ESL levels range from one, "entering"; to six, "reaching," according to WIDA. These values may be seen as being representative of a student's ability in the use and comprehension of language, ranging from a beginner to native level user of the English language. The values, while continuous, are not seen as interval data as each decimal represents a proportion within a proficiency level and not seen as equivalent to a decimal place within another proficiency level (ACCESS for ELLS, 2011).

In this sample, the lowest ESL proficiency level was 1.0 based on WIDA testing and the highest proficiency level was a 6.0. The mean was a $4.38(S D=1.61)$.

NAI Score. The NAI score is the scale used to identify the results of the NNAT, where 100 is set as the mean score similar to a traditional IQ test. Out of the sample of 203 students, the low score was a 73.0. The highest score was a 155. The average score out of this sample was a $108.41(S D=15.48)$.

\section{Examining the Hypotheses}

The hypotheses will be presented and tested following the methods outlined in chapter three and presented here in summary. After each hypothesis is presented, the corresponding data will be presented in table format and then explained to either accept or reject the null hypothesis. 


\section{Hypothesis One}

The first hypothesis stated that nonverbal aptitude as measured by the NAI score, the independent variable, accounts for a significant amount of unique variance in predicting academic achievement as measured by the second semester GPA, the dependent variable, when controlling for grade level, gender, primary language spoken, and ESL level as measured by WIDA at the secondary grade levels (6-12).

To test this hypothesis, a hierarchical regression analysis was conducted using achievement as the dependent variable, and all other variables as independent variables. The null hypothesis will be the regression coefficient of the NAI score equals zero, provided a well-fitted regression.

Table 3, the Hierarchal Regression Coefficient Table for Semester 2 GPA, shows the three steps of the hierarchal regression analysis. It included the NAI first, as it is seen as being the most influential on the GPA statistically, followed by Gender and Grade Level a student is entering. The Excluded Variables (Table 4) shows the variables not included in the regression model because they do not add unique variance potentially due to multi-collinearity, or the fact that the variables are so highly correlated that they essentially measure the same construct (Cohen \& Cohen, 1983). Table 5 shows us that

the Pearson correlation between the NAI Score and the $2^{\text {nd }}$ Semester GPA to be .276 , giving us an $R$ - squared value of .076, as seen in Table 3.

The final regression model at the third step on Table 3 shows the NAI regression coefficient was 0.323 , and it is significantly different from zero with a one-tail test that predicts direction $(p$-value $<0.0005)$ showing that NAI is a significant predictor of GPA on the 2 nd semester GPA. The amount of variance explained by the overall model was 
$15.5 \%$, which is a medium effect size (Cohen, 1988). To summarize, the analyses revealed that the null hypotheses must be rejected

Table 3

Hierarchal Regression Coefficient Table for Semester 2 GPA (Dependent Variable)

\begin{tabular}{|c|c|c|c|c|c|c|c|}
\hline \multirow{2}{*}{\multicolumn{2}{|c|}{ Step }} & \multicolumn{2}{|c|}{ Unstandardized Coefficients } & \multicolumn{3}{|c|}{ Standardized Coefficients } & \multirow{2}{*}{$\begin{array}{r}\text { Step } \\
R^{2}\end{array}$} \\
\hline & & $B$ & Std. Error & $\beta$ & $t$ & Sig. & \\
\hline \multirow[t]{2}{*}{1} & (Constant) & 1.957 & .276 & & 7.081 & $<.001$ & .076 \\
\hline & NAI Score & .010 & .003 & .276 & 4.055 & $<.001$ & \\
\hline \multirow[t]{3}{*}{2} & (Constant) & 2.062 & .273 & & 7.562 & $<.001$ & .119 \\
\hline & NAI Score & .010 & .002 & .280 & 4.206 & $<.001$ & \\
\hline & Sex & -.273 & .076 & -.207 & -3.111 & .002 & \\
\hline & (Constant) & 2.516 & .310 & & 8.128 & $<.001$ & .155 \\
\hline & NAI Score & .012 & .002 & .323 & 4.827 & $<.001$ & \\
\hline & Sex & -.295 & .077 & -.257 & -3.807 & $<.001$ & \\
\hline & Grade Entering & -.075 & .026 & -.202 & -2.920 & .004 & \\
\hline
\end{tabular}

Note: $N=203$

The change in $R^{2}$ from Step 1 to Step 2 is +.043 ; the change in $R^{2}$ from Step 2 to Step 3 is +.036 
Table 4

Excluded Variables

\begin{tabular}{|c|c|c|c|c|c|c|c|}
\hline \multirow[b]{2}{*}{ Model } & \multirow[b]{2}{*}{ Beta In } & \multirow[b]{2}{*}{$\mathrm{t}$} & \multirow[b]{2}{*}{ Sig. } & \multicolumn{4}{|c|}{ Collinearity Statistics } \\
\hline & & & & Partial Correlation & n |Tolerance & VIF & Min. Tolerance \\
\hline 1 Sex & -.207 & -3.111 & .002 & -.215 & 1.000 & 1.000 & 1.000 \\
\hline Grade Ent & -.135 & -1.954 & .052 & -.137 & .956 & 1.046 & .956 \\
\hline Nat Eng $\mathrm{S}$ & -.061 & -.897 & .371 & -.063 & 1.000 & 1.000 & 1.000 \\
\hline ESL Level & .032 & .467 & .641 & .033 & .999 & 1.001 & .999 \\
\hline Grade Ent & -.202 & -2.920 & .004 & -.203 & .894 & 1.119 & .894 \\
\hline Nat Eng S & -.063 & -.944 & .346 & -.067 & 1.000 & 1.000 & .999 \\
\hline ESL Level & .058 & .868 & .386 & .062 & .984 & 1.016 & .984 \\
\hline 3 Nat Eng $S$ & -.066 & -1.016 & .311 & -.072 & .999 & 1.001 & .894 \\
\hline ESL Level & .031 & .467 & .641 & .033 & .963 & 1.038 & .875 \\
\hline \multicolumn{8}{|c|}{ Note: Sex is coded $1=$ Male; Female $=0$} \\
\hline \multicolumn{8}{|c|}{ Native Eng. Speaker is coded $1=$ Yes; No $=0$} \\
\hline
\end{tabular}

In addition, it is seen statistically that as an average for each point increase in the NAI, the GPA will increase by 0.323 . The correlation between NAI and GPA was 0.276 (Table 5, Correlations Table) with a $p$-value $<0.0005$ ) corroborating the significant relation between NAI and GPA. 
Table 5

Intercorrelations among Research Variables

\begin{tabular}{|c|c|c|c|c|c|c|c|}
\hline & Sem & ester 2 GPA & Sex & Grade Entering & Native Eng. Speaker & ESL Level & NAI Score \\
\hline Pearson & Semester 2 GPA & 1.000 & -.201 & -.071 & -.058 & .040 & .276 \\
\hline \multirow[t]{5}{*}{ Correlation } & Sex & -.201 & 1.000 & -.244 & -.009 & .124 & .021 \\
\hline & Grade Entering & -.071 & -.244 & 1.000 & -.013 & -.160 & .211 \\
\hline & Native Eng Speaker & -.058 & -.009 & -.013 & 1.000 & .625 & .012 \\
\hline & ESL Level & .040 & .124 & -.160 & .625 & 1.000 & .029 \\
\hline & NAI Score & .276 & .021 & .211 & .012 & .029 & 1.000 \\
\hline \multirow{6}{*}{$\begin{array}{l}\text { Sig. } \\
\text { (1-tailed) }\end{array}$} & Semester 2 GPA & & .002 & .158 & .208 & .287 & .000 \\
\hline & Sex & .002 & & .000 & .451 & .039 & .384 \\
\hline & Grade Entering & .158 & .000 & & .429 & .011 & .001 \\
\hline & Native Eng Speaker & .208 & .451 & .429 & & .000 & .430 \\
\hline & ESL Level & .287 & .039 & .011 & .000 & & .340 \\
\hline & NAI Score & .000 & .384 & .001 & .430 & .340 & \\
\hline \multicolumn{8}{|c|}{ Note: Sex is coded $1=$ Male; Female $=0$} \\
\hline \multicolumn{8}{|c|}{ Native Eng. Speaker is coded $1=$ Yes; No $=0$} \\
\hline \multicolumn{8}{|c|}{$N$ Size $=203$} \\
\hline
\end{tabular}

To control for Type I error, or finding a false positive, the Bonferroni method was utilized. The Bonferonni method utilizes the alpha level, in this case .05 , and divides it by the number of tests for significance, which in this case is five. This leaves us with an adjusted alpha level of .01 and $p$ is the calculated value and has to be less than or equal to the adjusted alpha of .01 (Newman et al., 2000).

After running a general linear regression model and controlling for Type I error, Table 6 shows that Gender, Grade Entering, and the NAI are still statistically significant at the adjusted level of .001. This statistical model identified a .096 difference in the ability of the NAI to predict academic achievement over and above all of the other independent variables combined. 
Table 6

Regression Coefficient Table for Semester 2 GPA (Dependent Variable)

\begin{tabular}{lccccccc}
\hline Model Unstandardized Coefficients & Std. Coefficients & & & \\
& $B$ & Std. Error & $\beta$ & $t$ & Sig. & $R^{2}$ \\
& & & & & & \\
\hline 1 (Constant) & 3.363 & .273 & & 12.302 & $<.001$ & .072 \\
Sex & -.284 & .082 & -.248 & -3.475 & .0005 & \\
Grade Ent & -.040 & .027 & -.109 & -1.542 & .0645 & \\
Nat Eng & -.193 & .111 & -.155 & -1.742 & .0415 & \\
ESL Level & .053 & .032 & .150 & 1.659 & .0495 & \\
& & & & & & \\
\hline 2 (Constant) & 2.356 & .335 & -.270 & -3.977 & $<.001$ & \\
Sex & -.309 & .078 & -.186 & -2.655 & .0045 & \\
Grade Ent & -.069 & .026 & -.144 & -1.706 & .0450 & \\
Nat Eng & -.180 & .105 & .124 & 1.477 & .0750 & \\
ESL Level & .044 & .031 & .319 & 4.757 & $<.001$ & \\
NAI & .012 & .002 & & & & \\
\hline
\end{tabular}

Note: One-tailed test of significance

$N$ Size $=203$

$R^{2}$ change from Step 1 to Step 2 is .096

Sex is coded $1=$ Male; Female $=0$

Native Eng. Speaker is coded $1=$ Yes; No $=0$

\section{Hypothesis Two}

The second hypothesis states that there is a significant difference in nonverbal aptitude mean scores as measured by the NAI score between the genders. 
Hypothesis two was tested using a two tailed $t$-test. The null hypothesis was that the mean NAI score for males is not significantly different than the mean nonverbal academic ability score for females as measured by the NAI score.

The average NAI for Females was $108.16(S D=11.89)$ and for Males, it was $108.65(S D=18.26)$ as shown in Table 7. Levene's Test for Equality of variance did not support the underlying assumption of equal variance between the two groups. Because of this, the second row of Table 8 will be used to test the variance. The data provided in this row does not assume for equal variance. The t-test identifies a $p$-value equal to 0.822 showing that the difference between the means is most likely due to chance; hence, we fail to reject the null hypothesis and accept that gender did not make any significant difference at alpha point .05 on the NAI score in this study. The difference of NAI means between genders is 0.484 with a $95 \%$ confidence interval of $(-4.78,3.81)$ including the zero, as shown in Table 8 . This study does not identify a possible bias due to gender, and thus the second research hypothesis was not supported.

\section{Table 7}

Group Statistics for NAI Score Based Upon Sex

\begin{tabular}{llccc}
\hline Sex & $N$ & Mean & Std. Deviation & Std. Error Mean \\
\hline Male & 98 & 108.16 & 11.892 & 1.201 \\
Female & 105 & 108.65 & 18.256 & 1.782 \\
\hline
\end{tabular}


Table 8

Independent Samples $t$-Test for the NAI Score Based Upon Sex

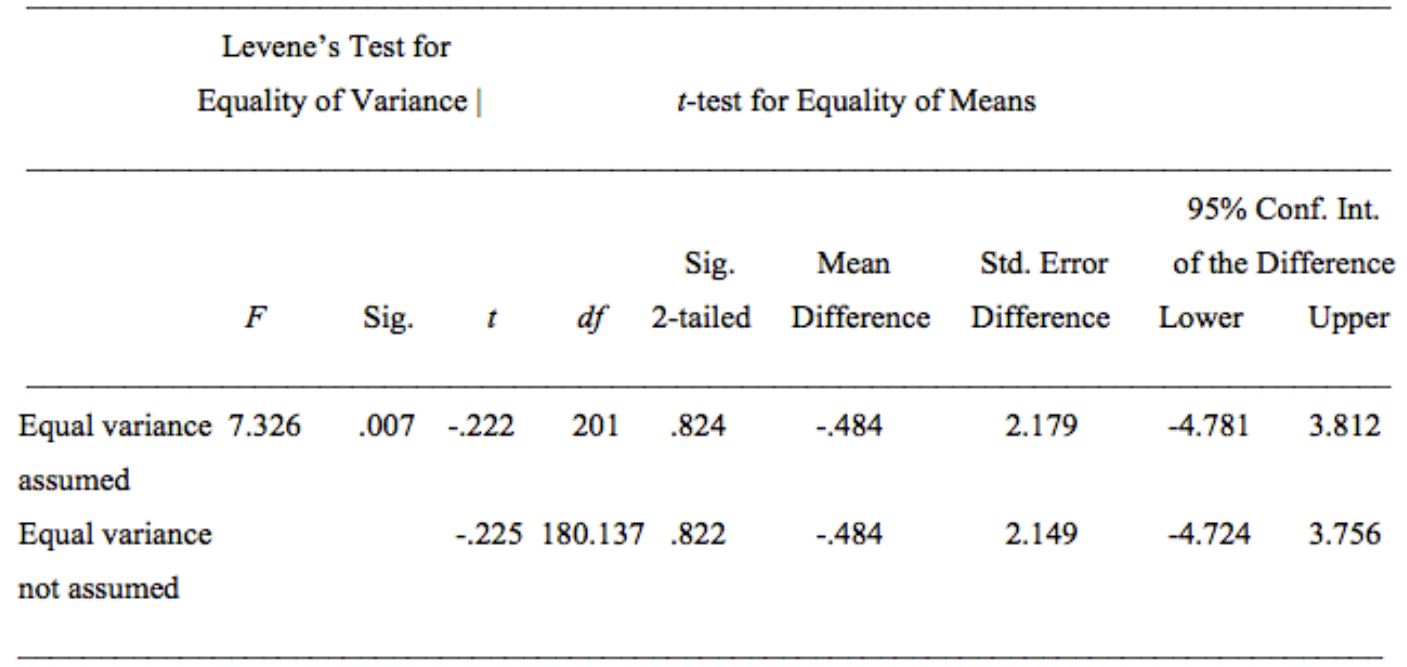

Note: $N$ Size $=203$

\section{Hypothesis Three}

The third hypothesis states that there is a significant difference in nonverbal aptitude mean scores as measured by the NAI score of non-native English speakers as compared to native speakers.

Hypothesis three was tested using a two tailed $t$-test. The null hypothesis was that the mean NAI scores for English as primary language students were not significantly different than the mean nonverbal academic ability scores for students with other than English as their primary languages.

The average NAI for non-native English speakers was $108.37(S D=14.43)$, and for native English speakers, it was $108.52(S D=17.76)$, as shown in Table 9. Levene's Test supports the underlying assumption of equal variance. Since this assumption is tenable and the $t$-test found no statistical difference in variance ( $p$-value $=0.950)$, as shown in table 10, the $t$-test failed to reject the null hypothesis. We must accept that 
being a non-native English speaker did not make any significant difference at alpha point .05 on the NAI score in this study. The difference of NAI means between native and non-native speakers is 0.15 with a $95 \%$ confidence interval of $(-4.81,4.52)$, including the zero as shown in Table 10. This study does not support a possible bias in determining nonverbal aptitude due to a student's ability to speak English as a native speaker; thus, the third research hypothesis was not supported.

Table 9

Group Statistics for NAI Score Based Upon Native English Speaking Ability

\begin{tabular}{llccc}
\hline Lang. & $N$ & Mean & Std. Deviation & Std. Error Mean \\
\hline NonNative & 141 & 108.37 & 14.429 & 1.215 \\
Native Eng & 105 & 108.52 & 17.761 & 2.256 \\
\hline
\end{tabular}


Table 10

Independent Samples t-Test for the NAI Score Based Upon Native English Speaking Ability

\begin{tabular}{|c|c|c|c|c|c|c|c|c|c|}
\hline \multicolumn{5}{|c|}{$\begin{array}{c}\text { Levene's Test for } \\
\text { Equality of Variance }\end{array}$} & \multicolumn{5}{|c|}{$t$-test for Equality of Means } \\
\hline & \multirow[b]{2}{*}{$F$} & \multirow[b]{2}{*}{ Sig. } & \multirow[b]{2}{*}{$t$} & \multirow[b]{2}{*}{$d f$} & \multirow{2}{*}{$\begin{array}{c}\text { Sig. } \\
\text { 2-tailed }\end{array}$} & \multirow{2}{*}{$\begin{array}{c}\text { Mean } \\
\text { Difference }\end{array}$} & \multirow{2}{*}{$\begin{array}{l}\text { Std. Error } \\
\text { Difference }\end{array}$} & \multicolumn{2}{|c|}{$\begin{array}{l}95 \% \text { Conf. Int. } \\
\text { of the Difference }\end{array}$} \\
\hline & & & & & & & & Lower & Upper \\
\hline $\begin{array}{l}\text { Equal variance } \\
\text { assumed }\end{array}$ & 0.000 & .996 & -.062 & 201 & .950 & -.147 & 2.364 & -4.810 & 4.515 \\
\hline $\begin{array}{l}\text { Equal variance } \\
\text { not assumed }\end{array}$ & & & -.058 & 97.946 & 6.954 & -.147 & 2.562 & -5.232 & 4.937 \\
\hline
\end{tabular}

Note: $N$ Size $=203$

\section{Chapter Summary}

The results of this study support hypothesis one. Aptitude, as measured by a nonverbal aptitude test, the NNAT in this study, demonstrates a positive Pearson correlation with academic achievement as measured by a new student's second semester GPA at the site in which this study was performed when controlling for the grade entering, the student's gender, ESL level as determined by WIDA testing, and whether the student is a native English speaker.

Hypotheses two and three looked to identify possible bias by the NAI in regards to students based upon gender as well as whether the students are native English speakers. In both of these instances, the null hypotheses identified no significant 
differences between the groups with the sample of this study. The $t$-tests failed to reject the null hypotheses, thus failing to identify potential bias in the NNAT for these groups. 


\section{CHAPTER V \\ DISCUSSION}

Chapter 5 presents a summary of the study to include a review of the hypotheses and the purpose behind the study. This will be followed by a discussion of the results as they pertain to each hypothesis. This will be followed by implications for theory, research, and practice as well as a discussion of the limitations of this study in generalizability.

\section{Summary of the Study}

Private schools are established to serve a purpose that may be delineated in its mission, vision, and/or objective statements. Admissions within a private school must work with its applicant pool to best identify the students from the applicant pool that it believes have the best opportunity for success within the school's programs.

Students applying to private schools may represent diverse backgrounds for a number of reasons. This may reflect diversity that already exists within the United States and the community in which a private school is located; but is also seen by the increase in international students coming to the United States to study (Open Doors, 2015). The diversity of students applying to private schools represents numerous cultures, subcultures, customs, and languages. The differences that diverse students bring with them may highlight, promote, and strengthen differing intelligences within students (Verney et al., 2005). Additionally, a language other than English may be spoken as the students' primary language. These differences may pose a challenge to educators seeking to quantify the students' academic aptitude. This may make it difficult to properly place 
them into appropriate academic programs that will allow students to be properly challenged and grow.

Consequently, it is increasingly important that admission directors be familiar with the population they serve. This familiarity will help to ensure their practices are non-discriminatory in predicting student ability to achieve academically within a school, apart from demographic differences. According to the SSATB, this will include knowledge of the general population of the community, the private school market, area competition to include both public and private schools, as well as the school itself and its mission.

Standardized cognitive ability tests may be used to serve students in numerous ways. Depending upon how they are used, however, they may also have a detrimental impact on students as the results may be inappropriately used to place students in educational programs that do not meet the students at their need. Studies have shown that there is a disproportionately high number of minority students in special education programs (MacMillan et al., 1993) as well as a disproportionately low number placed into gifted programs (Ford-Harris, 1993). This may be due, in part, to the use of standardized tests for placement and the failure to understand the detrimental impact that biases within the test may hold for certain students they are testing, such as students who are not of the majority; non-Hispanic Whites, or do not have the same ability with the English language as might be expected (Valencia \& Suzuki, 2001).

In addition to working to reduce bias in testing based upon differences in culture and/or race, one must be cognizant of any bias that may exist based upon gender. Differences alone in scores may not identify bias. They may also be due to variables not 
included in a study such as work ethic or motivation; however, they should not be ignored (Meade \& Fetzer, 2009).

Previous research has implied that a measurement of general intelligence may not require both verbal and non-verbal components. Removing the verbal component may render it a more accurate predictor by reducing bias when working with a diverse population (Naglieri \& Ronning, 2000).

ETS has identified nonverbal tests as being useful for testing students, whose primary language is not English, to reduce bias (Nonverbal Aptitude, 1993). Nonverbal tests are already utilized in numerous schools around the nation to identify students for gifted programs. They are often used by identifying cut off scores to identify students for placement into these gifted programs (Giessman et al., 2013).

The Naglieri Nonverbal Ability Test (NNAT), currently in its second edition, was developed to measure general ability with nonverbal measurements in the form of progressive matrices, or picture puzzles that challenge one's logic with intricate patterns. This was done to promote fairness regardless of gender, race, and ethnicity (Directions for Administration 2011).

The results are given with a score called the Naglieri Academic Index (NAI). Using inferential norming and conversions to standard scores, the mean score on the NNAT2 or the NAI score is 100 with a standard deviation of 16 and a range of 40-160 (Technical Information, 2011).

The researcher of this study, based upon the arguments in favor of utilizing a nonverbal aptitude test in measuring general intelligence, produced the following hypotheses. 
1. Nonverbal aptitude as measured by the NAI score, the independent variable, accounts for a significant amount of unique variance in predicting academic achievement as measured by second semester GPA, the dependent variable, when controlling for grade level, gender, primary language spoken, and ESL level as measured by WIDA at the secondary grade levels (6-12).

2. There is a significant difference in nonverbal aptitude mean scores as measured by the NAI at the secondary grade levels (6-12) by or between the genders.

3. There is a significant difference in nonverbal aptitude mean scores as measured by the NAI of non-native English speakers as compared to native speakers at the secondary grade levels (6-12).

To determine the ability of nonverbal aptitude testing to predict academic achievement, an ex post facto study with hypotheses that controlled for viable alternative explanations was conducted as a small private secondary school in South Florida. All new applicants who applied to the school for grades 6 through 12 were given the Naglieri Nonverbal Aptitude Test; an ESL placement test, WIDA; as well as other components of the school's admissions testing prior to acceptance. The GPA of accepted students was collected from their second semester at the school. All needed data on the new students from three academic years, 2013-14, 2014-15, and 2015-16, were collected to obtain as large a sample size as possible. The necessary data exist from these years and includes the students' NAI score, second semester GPA, grade level entering the school, gender, whether the student is a native English speaker, and what ESL level they are as measured by WIDA. An $n$ size of 203 students was achieved by pulling three years' worth of data. A correlational analysis was then done to identify whether a positive relationship existed 
between the results of the nonverbal test, the NAI score prospective students obtained, and the GPA they earned during their second semester at the school. A hierarchical regression analysis was conducted using achievement as the dependent variable, and all other above mentioned variables as independent variables to identify a possible relationship. Differences of the mean NAI scores between male and female students as well as between native and non-native English speakers were analyzed to identify whether a difference across the means was identified between these groups. Hypotheses two and three were tested using $t$-tests.

This study was undertaken because there is insufficient research and thus a knowledge gap with regards to the link between nonverbal test performance and student achievement. Admissions departments within schools, particularly private schools, often require a predictive assessment to identify a student's potential to find success in a rigorous academic environment (SSATB: Home, n.d.). Numerous tests are available. For the majority, however, student success relies on a high functioning knowledge of the English language (Lakin \& Lai, 2012). Additionally, a common culture is often assumed by the test creators in regards to the test takers (Greenfield, 1997). With increased mobility of students around the globe, this puts many of these international students at a disadvantage when faced with taking these tests. For this reason, nonverbal test performance should be studied in the context of private school settings in order to identify its relationship with academic achievement.

Since previous studies have shown that traditional IQ and aptitude testing may present bias in testing and placing students into appropriate programs (Verney et al., 2005), nonverbal testing may better gauge student aptitude within a diverse population. 
This is due to the purported reduction of bias in nonverbal testing (Naglieri \& Ford, 2003).

This study will look to determine whether nonverbal aptitude as measured by a nonverbal aptitude test accurately predicts academic achievement as measured by second semester grade point average (GPA) in a private secondary school setting while controlling for gender, grade level, whether the student is a native English speaker, and English as a Second Language (ESL) level. If nonverbal aptitude as measured by a nonverbal aptitude test demonstrates this ability, then it would be seen as fit for admission purposes to be used in conjunction with other indicators as identified by an admissions department. The study also aims to see if the mean results are comparable across differing demographic factors to include gender, and whether the student is a native English speaker. If a difference across the means is identified in any of these groupings, further research may be necessary to assess whether test bias exists against any of these groups.

Results suggest that nonverbal aptitude as measured by the NNAT and utilizing the NAI does have a positive correlation with academic success as measured by second semester GPA. This suggests that the NAI can be utilized in an admissions process to improve the ability to predict success while reducing testing bias toward diverse students. Furthermore, the sample of this study did not show a significant difference of the means between the genders or the native and non-native English speaker, further supporting its use to reduce bias in the admissions process. 


\section{Discussion of the Results}

Guided by both the theoretical base for this study and the current research that was done, this section will discuss the results of each of the hypotheses tested. The results of this study were statistically significant and may make a meaningful contribution to theory, research, and practice. A brief summary of the findings will conclude this section.

\section{Hypothesis One}

The first hypothesis stated that the NAI score a student receives based upon his or her performance on the NNAT accounts for significant and unique variance in predicting academic achievement as measured by the second semester GPA. In running a hierarchal regression model, the grade level, the gender, the ESL level as measured by WIDA, and whether the student is a native English speaker were variables that were controlled for. Results indicate that the NAI is a significant predictor of GPA in the $2^{\text {nd }}$ semester, thus rejecting the null hypothesis. The Pearson correlation identified in this study between the NAI and the second semester GPA that a student earned was approximately $27.6 \%$.

An additional post hoc analysis was performed using simultaneous general linear regression testing of a full model versus a restricted model to identify unique variance in predicting academic achievement as measured by the second semester GPA. In the full model, all control variables in this study were utilized, whereas in the restricted model, the NAI was pulled out. In doing this, it is found that the NAI accounted for .096, or approximately $10 \%$ of the unique variance in achievement while controlling for gender, grade entering, ESL level, and whether a student is a native English speaker. The results 
of this post hoc analysis can be found in Table 11 and can be extracted by subtracting the R Square for model 1 from model 2.

\section{Table 11}

Full and Restricted Model Statistics for Hypothesis One

\begin{tabular}{llll}
\hline Model & $R$ & $R$ Square & Adj. $R$ Square \\
\hline 1 (Restricted) & .269 & .072 & .054 \\
2 (Full) & .168 & .168 & .147 \\
\hline
\end{tabular}

Note: The restricted model includes ESL Level, Sex, Grade Entering, and Native English Ability. The full model includes NAI score as well.

$N$ Size $=203$

Brody (1992) told us that the most important dimension of validity for an ability test is its ability to predict school achievement. At the same time, Allan (2005) has told us that factors such as motivation and family involvement amongst other variables also have a relationship with school achievement. Keeping these two things in mind, one would not expect a perfect correlation between nonverbal aptitude as measured by the NAI and academic achievement as measured by second semester GPA. One would expect, however, a modest correlation if the NAI were to be utilized in practice based upon the results of studies similar to this one. A .276 Pearson Correlation is similar to what is found between the SAT and fGPA of college students (McDonald et al., 2001; Sackett et al., 2009). This would seemingly support Naglieri's (2006) claim that nonverbal aptitude testing is able to gauge general aptitude as well as aptitude testing that does utilize language. It would also seem to be in line with previous research by Balboni, 
Naglieri, and Cubelli (2010) and Naglieri and Ronning (2000), who came to a similar conclusion.

\section{Hypothesis Two and Three}

The second hypothesis states that there is a significant difference in nonverbal aptitude means scores as measured by the NAI score between the genders. The null hypothesis thus states that there is no significant difference in the means of the NAI between the genders.

This study was unable to reject the null hypothesis, as there was no statistical difference identified in the means of the two genders. The difference of the means was only 0.49 , well within the confidence interval of $95 \%$.

The third hypothesis states that there is a significant difference in nonverbal aptitude mean scores as measured by the NAI score of non-native English speakers as compared to native speakers. The null hypothesis thus states that there is no significant difference in the means of the NAI between these two groups.

Similarly to the results of the second hypothesis, this study was unable to reject the null hypothesis, as there was no statistical difference identified in the means of these two groups. The difference of the means was only 0.15 , again, well within the confidence interval of $95 \%$.

The NNAT purportedly reduces test bias based upon gender as well as cultural and linguistic differences (Naglieri \& Ford, 2003). Hypotheses two and three looked to analyze these claims and see whether the results of this study would suggest that a bias may in fact exist with the NNAT due to gender or cultural differences as identified by the primary language that the students speak. Numerous studies have identified test bias 
based upon gender in traditional standardized tests (Department for Education and Skills, 2007; Kirsch et al.,, 2002; Mullis et al., 2003; Mullis et al., , 2007; Guion, 1998). Numerous studies have also identified test bias based race and/or culture (Camara \& Schmidt, 1999; Huggins, 2014; Suzuki \& Gurkin, 1993; Whitworth \& Gibbons, 1986). The findings of this study support the claims made by Naglieri and Ford (2003) as there is not a statistically significant difference in means amongst these various groups found within the sample of this study.

\section{Implications for Theory}

This study sought to identify the predictability of second semester GPA that a new student would earn at a new school by the NAI. This was done taking into account Bandura's (2002) SCT. SCT recognizes that a person, his or her environment (people, organizations, and physical characteristics), and internal processing of information all have an impact on one another and play a significant role in the development of cognition in a person - triadic reciprocity. Accepting this theory then as a foundation for this study means that one would not expect a perfect correlation between the NAI and academic achievement made during the second semester in attendance at a new school. It recognizes that the environment will play a role in their achievement; their aptitude and personal processing of the stimuli around them also have significance. The results of this study support this theory as the Pearson Correlation between the NAI and second semester GPA is of modest or medium power (Cohen, 1988). The unique variance attributed to the NAI, or nonverbal aptitude, is approximately $10 \%$. This signifies the fact that there are other important constructs to measure that may be internal as well as 
external for a student in order to predict academic achievement at a higher level, as would be expected by SCT.

Pajares (2002) summarizes the findings of psychologists and states that both nurture and nature play a role in shaping a person. This follows the SCT theory as identified by Bandura (2002) and is also supported by the findings of this study, that the NAI accounts for only $10 \%$ of unique variance when controlling for gender, the grade a student is entering, whether the student is a native English speaker, and ESL level in predicting the second semester GPA by the NAI.

Recognizing that non-verbal intelligence as measured by the NNAT may be used as part of a process to predict academic achievement does not give further clarity to the definition of aptitude. Lohman (2005) and Naglieri and Ford (2005) are at odds with each other as they attempt to define it. Lohman would argue that one's prior knowledge helps to define aptitude, whereas Naglieri and Ford would argue that aptitude, as seen as potential, is the innate ability to learn that is not determined by prior knowledge. The ability of the NAI to predict academic achievement attempts to gauge this innate ability regardless of background knowledge to include language ability. The results of this study showed no statistically significant difference in proficiency in English in determining achievement level obtained. This study then supports Naglieri and Ford, at least in a limited capacity.

As previously indicated in this research, the $g$ factor is commonly incorporated into definitions of intelligence. Nunnally (1967) demonstrated that if indeed a $g$ factor exists and is common to a variety of tests, it is therefore sufficiently accurate to be used to determine the correlation between tests exhibiting the $g$ factor. Hispanics have been 
routinely found, on average, to score approximately one half standard deviation below White Americans, while Black Americans routinely score one full standard deviation below White Americans on average on standardized tests. Asian Americans, on the other hand, have been found, on average, to score half a standard deviation above White Americans (Camara \& Schmidt, 1999; Suzuki \& Gurkin, 1993; Whitworth \& Gibbons, 1986).

Current testing, however, often shows significant bias on standardized tests in favor of those with a common culture and language with the tests' creators (Jensen, 1980; Neisser et al., 1996). Bias on standardized tests opens room for a discussion concerning both the test variety administered in determining the $g$ factor and whether the majority of standardized tests exhibit similar bias toward students of distinct backgrounds from the authors of the tests. Ceci (1990) stated that narrowing general intelligence down to just a few abilities, commonly done on standardized tests, has been seen as misleading as it may test certain knowledge emphasized by a particular culture.

The results of this study may bring into question the $g$ factor and its association with possible racial bias inherent in intelligence measures. Approximately $70 \%$ of the participants in this study were non-native English speakers. Of the students in the school, $82 \%$ were Hispanic. Based upon these statistics and the race relationship of the $g$ factor to race (Rushton \& Jensen, 2003; Spearman, 1927), it would be reasonable to expect that the mean score on the NAI from this sample would be less than the overall mean for everyone combined established by the test's normative data. Notwithstanding, the sample of this study had a mean score of 108 , higher than the mean of the normative data 
indicating that the measure, at least in the case of this research, was not biased by race as claimed by Naglieri and Ford (2003).

This study would then support the more recent trends of psychologists in identifying more ability categories to identify ability such as Carroll in 1993 that identified 70 such abilities or gauging aptitude though testing that minimizes test bias.

The Cattell-Horn-Carroll theory as postulated by McGrew and Flanagan (1998) identified verbal IQ as a form of crystallized intelligence. Horn and Cattell (1966) saw crystallized intelligence as one that could be developed. They also recognized a fluid intelligence that allowed one to solve novel problems and utilize all other intelligences in application. In line with Carroll (1993), Horn and Cattell saw fluid intelligence as fixed or innate while crystalized intelligence could be developed. McGrew and Flanagan defined nonverbal IQ a measure of numerous constructs of fluid intelligence. These theories then would in fact support the hypothesis that a higher nonverbal IQ, such as nonverbal aptitude as measured by the NAI, would in fact predict a higher level of academic achievement. Once again, the findings of this study would support this theory while accepting that nonverbal aptitude is not the sole determinant of academic achievement.

\section{Implications for Research}

While this study has shown that nonverbal aptitude as measured with the NAI may be utilized in the prediction of academic achievement, there are clearly a number of questions that this study points to that are not well defined or answered.

Regarding the two definitions or ideas behind the term aptitude as presented in this study, the question may be asked, "Is prior knowledge or academic potential a better 
predictor of academic achievement?" If one is asking this question, it may lead to other questions that are pertinent in practice.

This study identifies theory of intelligence that categorizes intelligence into both fluid and crystallized intelligences. Fluid intelligence is seen as an overarching intelligence that other intelligences are dependent upon to put knowledge to work (McGrew \& Flanagan, 1998). The NNAT is utilized in this study as an instrument to gauge this intelligence. It is referred to as "general intelligence" by the creators of the NNAT (Directions for Administration, 2011). While the NNAT has a format of its own that takes the form of a multiple-choice test, the question might be asked, "Does the NNAT still reflect test bias against cultures that are not familiar with this type of test or against cultures in which 'traditional' academics are not the norm?" Because the test seeks to reduce bias in minority groups, it would be important to know whether certain cultures, due to vast differences from the American White majority, would be unable to have their innate aptitude or general intelligence measured by a paper and pencil test. If this is the case, what is another method for identifying fluid intelligence?

This study identifies the ability of the NNAT to predict academic achievement. While significant, however, the amount of unique variance attributed to the NNAT through the NAI is approximately $10 \%$. This figure tells us that there are many more contributing factors to a student's academic success or achievement. An analogy may better help to understand the significance. If one were to play the stock market, the ability to better predict market gains by $10 \%$ would provide great economic potential. Following Bandura's (2002) SCT, one may ask what internal and external factors also contribute to academic achievement and how might they be measured. 
The findings of this research should be tested again beyond the limits of one particular school. Schools may reflect populations that are more agrarian, suburban, or urban in location. They may include students of different cultures, races, and ethnicities. They may also have students of more varied SES. The school itself may be structured differently depending upon state as well as whether it is a public school, charter school, secular private, or religious school. Different regions within the United States may stress different cultural norms. All of these factors may have an impact on the student bodies they serve. A more diverse sample across these types of schools should also be tested to verify the results and give practitioners in education a more solid base for allowing the results to be used in making decisions within schools.

\section{Implications for Practice}

This study may have implications on schools for a number of different reasons. and may affect admissions in many different ways. First and foremost, schools may agree with the underlying assumption of this study that seeking to diversify the students accepted into a private school is beneficial to a program. If that is the case, then a school may scrutinize their admissions processes to determine whether the bias presented toward students is reduced whenever and wherever possible. This includes determining whether their testing process accepts a presubscribed background knowledge or looks for potential when deciding whether or not to accept a student. The decision to focus on background knowledge or aptitude would potentially change the testing procedure that is utilized within admissions. If a school chooses to focus on aptitude, what will the school do to place students into appropriate classes if their expected background knowledge needs to 
be built up to place students into equivalent classes with their peers as quickly as possible?

To better understand how the results of this study may be utilized, the school in which this study was performed and their use of the NNAT will be reviewed. The school serves a community that is a minority-majority community. In turn, the applicant pool to the school replicates this trend. The school seeks to serve the community and meet the community at its need. For this reason, it utilizes the NNAT to reduce bias in its application process.

The NNAT is utilized to measure a student's aptitude, but is only one part of the applicant screening process. Additionally, they take the ACT Engage to measure academic behaviors, social behaviors, and academic support in the home, amongst other constructs that may influence academic achievement. They have a personal interview with the admissions office, and take placement tests in English and in math.

The results of the NNAT, the ACT Engage, as well as the interview are used to determine whether a student will be accepted at the discretion of the admissions department. Students who want to do well and show a minimum ability to do well are accepted into the student body; diversity in demographics in the classroom is seen as beneficial as it brings more experiences into the classroom via the students who become part of the learning environment for all.

The placement or diagnostic tests, along with prior classes taken and passed, are used to identify the programs the school offers that will best meet the students' needs and allow them progress as far as possible in their time at the school. In math, students are able to take math courses that are traditionally two years behind a current student's grade 
level. These courses that work with students who are behind are intended to prepare students to progress through Algebra II in high school at a minimum. Students who are advanced are offered math courses that progress through AP Calculus BC and AP Statistics. Students who are identified as needing ESL support are provided ESL instructional and support time. Students with little to no English exposure are offered an English Language Learner (ELL) program in which for four hours a day, they are in an extensive program designed to immerse students in English in all of their core subjects while providing language support simultaneously. This is done in small group setting with no more than eight students. Remedial writing classes are also provided for students who are weak in this area. English classes progress through AP classes as well. For advanced students, dual enrollment classes with three different universities are also available. With all of these different programs available, one will find there is a constant push for students to move well beyond their current levels.

A school that seeks to utilize this study in a similar way may need to run its own pilot study of the NNAT when considering the limitations of this study to generalizability as presented next. This is because the demographics of the sample as well as the setting and its culture may be vastly different from other settings, preventing the replicability of this study in differing settings.

\section{Limitations of the Study to Generalizability}

While this study may provide an impetus for future research, its findings may not be generalizable to the public. First, the study is an ex post facto study of existing data; therefore, causality cannot be assumed. Secondly, this study used a convenience sample obtained at one school over the course of three academic school years. While the sample 
includes students from numerous countries, the majority of the sample is Hispanic. Some may consider the sample itself then to be relatively homogenous despite the fact that the students' countries of origin represent the majority of Latin American countries as well as Spain and Portugal. Additionally, the school is a religious school that more than likely attracts students with a similar religious belief. The school is situated in an affluent community and charges a tuition that many would consider to be prohibitive for a large portion of the population in the United States as well as some in the community it serves. It seeks to provide financial aid based upon need, but this will still reduce the number of applicants to the school.

Language bias, while reduced on the NNAT, may present itself through the test administrator. While the test itself provides instructions through graphical directions, some directions are still given by the test administrator. The school is able to accommodate English and Spanish speakers readily. Students who primarily speak a different language may be more challenging to present with comprehensive test taking instructions.

In looking at academic achievement, another limitation that may be present is grade inflation. Students who were overly concerned with their GPA may have taken courses that were seen as easier and did not challenge the students academically as more advanced courses would have. The incentive given for taking advanced courses, a .5 boost per class to their grade point average, may have limited this impact, but it is unknown.

The format of the NNAT itself is multiple-choice. The assumption is also made that students from diverse backgrounds are familiar with this type of test. This may not 
be the case as the school is not familiar with all cultures, school systems, and their differing practices.

For these reasons, one must weigh the sample of this study as well as its findings with the population one serves. Due to differences in population such as SES, cultural, religious, or linguistic differences, the findings of this study may not be replicated with a population reflecting different demographic traits.

\section{Conclusion}

The admissions department within a school plays a vital role in helping to create a diverse student body that reflects the community it serves. The admissions process can work to provide opportunity to the students of the community and seek to elevate the educational opportunities available to all by evaluating potential students by their aptitude or potential rather than prior learning or culture. It can be a process in which diversity is promoted and becomes additive to the culture and environment of the school as students from diverse backgrounds can bring their culture, their experiences, and their backgrounds to the classroom, and students are able to learn from each other.

The NNAT is a valuable tool that can be used to help evaluate student aptitude. This study suggests that it can contribute to the admissions process in predicting academic success. In conjunction with a process that evaluates the whole student, academically for diagnostic purposes, socially, and personally, students can be placed into programs that will encourage growth and provide a challenge to them to meet their potential. This researcher would encourage those who work in the admissions process within schools to examine their practices to ensure they are reaching out to the 
community they serve, and not only to the majority, by incorporating practices into their admissions process that are not inherently biased. 


\section{REFERENCES}

ACCESS for ELLs interpretive guide for score reports. (2011). WIDA Consortium. Retrieved from https://www.wida.us/assessment/ACCESS/ScoreReports/ ACCESS_Interpr+etive_Guide11.pdf

Allan, J. (2005). Grades as valid measures of academic achievement of classroom learning. The Clearing House, 78, 218-223.

Anderson, S., Medrich, E., \& Fowler, D. (2007). Which achievement gap? Phi Delta Kappan, 7, 547-550.

Artiles, A. J., Kozelski, E. B., Trent, S. C., Osher, D., \& Ortiz, A. (2010). Justifying and explaining disproportionality: A critique of underlying views of culture. Exceptional Children, 76, 279-299.

Balboni, G., Naglieri, J. A., \& Cubelli, R. (2010). Concurrent and predictive validity of the Raven Progressive Matrices and the Naglieri Nonverbal Ability Test. Journal of Psychoeducational Assessment, 28, 222-235. doi:10.1177/0734282909343763

Bandura, A. (1989). Social cognitive theory. In R. Vasta (Ed.), Annals of Child Development, 6, 1-60.

Bandura, A. (1997). Self-efficacy: The exercise of self-control. New York, NY: Freeman.

Bandura, A. (2002). Social cognitive theory in cultural context. Applied Psychology: An International Review, 51, 269-290.

Bliss, S. (2008). Concurrent and predictive validity of the Universal Nonverbal Intelligence Test-Group Ability Test (Unpublished doctoral dissertation). The University of Tennessee, Knoxville.

Brody, L. R. (1997). Gender and emotions: Beyond stereotypes. Journal of Social Issues, 53, 369-393. doi:10.1111/j.1540-4560.1997.tb02448.x

Brody, N. (1992). Intelligene. San Diego, CA: Academic Press.

Burney, V. H. (2008). Applications of social cognitive theory to gifted education. Roeper Review, 30, 130-139. doi:10.1080/02783190801955335

Camara, W. J., \& Schmidt, E. (1999). Group differences in standardized testing and social stratification. New York, NY: The College Board. Retrieved from https://research.collegeboard.org/sites/default/files/publications/2012/7/researchre port-1999-5-group-differences-standardized-testing-social-stratification.pdf 
Carman, C. A., \& Taylor, D. K. (2010). Socioeconomic status effects on using the Naglieri Nonverbal Ability Test (NNAT) to identify the gifted/talented. The Gifted Child Quarterly, 54, 75-84. doi:10.1177/0016986209355976

Carroll, J. (1993). Human cognitive abilities: A survey of factor-analytic studies. Cambridge, United Kingdom: Cambridge University Press.

Ceci, S. J. (1990). On intelligence . . more or less: A bioecological treatise on intellectual development. Englewood Cliffs, NJ: Prentice Hall.

Ceci, S. J., Rosenblum, T. B., \& Kumpf, M. (1998). The shrinking gap between high- and low-scoring groups: Current trends and possible causes. In U. Neisser (Ed.), The rising curve (pp. 287-302). Washington, DC: American Psychological Association. doi:10.1037/10270-010

Cognitive Abilities Test. (n. d.). Retrieved from http://www.hmhco.com/hmhassessments/ability/cogat-6

Cohen, J. (1988). Statistical power analysis for the behavioral sciences $\left(2^{\text {nd }}\right.$ ed.). San Diego, CA: Academic Press.

Cohen, J., \& Cohen, P. (1983). Applied multiple regression/correlation analysis for the behavioral sciences. Hillsdale, NJ: Erlbaum.

Côté, J. E., \& Levine, C. G. (2000). Attitude versus aptitude: Is intelligence or motivation more important for positive higher-educational outcomes. Journal of Adolescent Research, 15, 58-80.

Davies, A. (2011). Does language testing need the native speaker? Language Assessment Quarterly, 8, 291-308.

Deary, I. J., Strand, S., Smith, P., \& Fernandes, C. (2007). Intelligence and educational achievement. Intelligences, 35, 13-21.

Department for Education and Skills. (2007). Gender and education: The evidence on pupils in England. Retrieved from http://www.ttrb3.org.uk/gender-and-educationthe-evidence-on-pupils-in-england/

DeThome, L., \& Schaefer, B. (2004). A guide to child nonverbal IQ measures. American Journal of Speech-Language Pathology, 13, 275-290.

Directions for administration: Naglieri Nonverbal Ability Test, Second Edition. (2011). Upper Saddle River, NJ: NCS Pearson. 
Drake, K. (2006). Gifted services identification report. Saint Paul Public Schools, Department of Research, Evaluation and Assessment, Office of Research and Development.

Duckworth, A. L. (2006). Intelligence is not enough: Non-IQ predictors of achievement (Doctoral dissertation). Retrieved from PsycINFO. (Order No. AAI3211063.)

Dweck, C. S. (2010). Even geniuses work hard. Educational Leadership, 68, 16-20.

Elert, G. (1992). The SAT aptitude or demographics? Retrieved from https:/www.hypertextbook.com/eworld/sat.shtml

Ford, D. Y. (2012). Culturally different students in special education: Looking backward to move forward. Exceptional Children, 78, 391-405.

Ford-Harris, D. (1993). The underrepresentation of minority students in gifted education: Problems and promises in recruitment and retention. Journal of Special Education, 32, 4-14.

Furnham, A. (2014). Increasing your intelligence: Entity and incremental beliefs about the multiple "intelligences." Learning and Individual Differences, 32, 163-167. doi: 10.1016/j.lindif.2014.03.001

Gardner, H. (1983). Frames of mind: The theory of multiple intelligences. New York, NY: Basic Books.

Giessman, J. A., Gambrell, J. L., \& Stebbins, M. S. (2013). Minority performance on the Naglieri Nonverbal Ability Test, Second Edition, versus the Cognitive Abilities Test, Form 6: One gifted program's experience. Gifted Child Quarterly, 57, 101-109. doi:10.1177/0016986213477190

Green, S. (1991). How many subjects does it take to do a regression analysis? Multivariate Behavioral Research, 26, 449-510.

Greenfield, P. M. (1997). You can't take it with you. American Psychologist, 52, 11151124.

Grinberg, E. (2011). White children in the minority in 10 states. This Just In - CNN.com Blogs. Retrieved from http://news.blogs.cnn.com/2011/04/06/White-children-inthe-minority-in-10-states/.

Guion, R. (1998) Assessment, measurement, and prediction for personnel decisions. Mahwah, NJ: Erlbaum. 
Harvey, B. \& Klinger, J. (2014). Why are so many minority students in special education? Understanding race and disability in schools. $2^{\text {nd }}$ Edition. New York, NY: Teachers College Press.

Horn, J., \& Cattell, R. (1966). Refinement and test of the theory of fluid and crystallized general intelligences. Journal of Educational Psychology, 57, 253-270.

Huggins, A. C. (2014). The effect of differential item functioning in anchor items on population invariance of equating. Educational and Psychological Measurement, 74, 627-658.

Jensen, A. R. (1980). Bias in mental testing. New York, NY: Free Press.

Kaplan, R. M. \& Saccuzzo, O. P. (2009). Psychological testing: Principles, applications, and issues. Belmont, CA: Wadsworth.

Kaya, C. (2015). Internal validity: A must in research designs. Educational Research and Reviews, 10, 111-118.

Kerlinger, F. N. \& Lee, H. (2000). Foundations of behavioral research $\left(4^{\text {th }}\right.$ ed.). Fort Worth, TX: Harcourt.

Kirsch, I., de Jong, J., Lafontaine, J., McQueen, J., Mendelovits, J., \& Monseur, C. (2002). Reading for change: Performance and engagement across countries. Results from PISA 2000. Paris: OECD Retrieved from http://www.oecd.org/edu/school/programmeforinternationalstudentassessmentpisa /33690904.pdf

Kokhan, K. (2013). An argument against using standardized test scores for placement of international undergraduate students in English as a Second Language (ESL) courses. Language Testing, 30, 467-489. doi:10.1177/0265532213475782

Lakin, J. M. (2012). Assessing the cognitive abilities of culturally and linguistically diverse students: Predicting validity of verbal, quantitative, and nonverbal tests. Psychology in the Schools, 49, 756-769. doi:10.1002/pits.21630

Lakin, J. M., \& Lai, E. R. (2012). Multigroup generalizability analysis of verbal, quantitative, and nonverbal ability tests for culturally and linguistically diverse students. Educational and Psychological Measurement, 72, 139-158. doi:10.1177/0013164411408074

Lewis, J. (2001). Proceedings from Growing Partnerships for Rural Special Education '01: Language isn't needed: Nonverbal assessments and gifted learners. San Diego, CA. 
Lohman, D. F. (2005). Review of Naglieri and Ford (2003): Does the Naglieri Nonverbal Ability Test identify equal proportions of high scoring White, Black, and Hispanic students? The Gifted Child Quarterly, 49, 19-29.

Lopez, N. (n. d.). Mathematical notation comparisons between U.S. and Latin American countries. Todos: Mathematics for all. Retrieved from http://www.csus.edu/indiv/o/oreyd/ACP.htm_files/TODOS.operation.description. pdf

Lovie, P. (2005). Spearman, Charles, Edward. Encyclopedia of statistics in behavioral science. doi:10.1002/0470013192.bsa634

Manos, G. (2011). Construct validity of the Naglieri Nonverbal Ability Test for Black and White second grade students. Mankind Quarterly, 51, 269-287.

Mackintosh, N., \& Bennett, E. (2005) What do Raven's Matrices measure? An analysis in terms of sex differences. University of Cambridge, Department of Experimental Psychology. doi:10.1016/j.itell.2005.03.004

Macklem, G. L. (1990). Measuring aptitude. Practical Assessment, Research \& Evaluation, 2. Retrieved from http://PAREonline.net/getvn.asp?v=2\&n=5

MacMillan, D., Gresham, F., \& Sipersein, G. (1993). Conceptual and psychometric concerns about the 1992 AAMR definition of mental retardation. American Journal on Mental Retardation, 98, 325-335.

McCallum, R., Bracken, B., \& Wasserman, J. (2001). Essentials of nonverbal assessment. New York, NY: Wiley.

McGrew, K., \& Flanagan, D. (1998). The intelligence test desk reference: Gf-Gc crossbattery assessment. Boston, MA: Allyn \& Bacon.

McDonald, A. S., Newton, P. E., Whetton, C., \& Benefield, P. (2001) Aptitude testing for university entrance: A literature review. The Sutton Trust. Retrieved from http://www.nfer.ac.uk/publications/APL01/APL01_home.cfm

Meade, A. \& Fetzer, M. (2009) Test bias, differential prediction, and a revised approach for determining the suitability of a predictor in a selection context. Organizational Research, Methods, 12, 738-761. Retrieved from http://orm.sagepub.com/content/early/2009/02/06/1094428109331487.abstract

Mullis, I., Martin, M., Gonzalez, E., \& Kennedy, A. (2003). PIRLS 2001 international report: IEA's study of reading literacy achievement in primary school in 35 countries. Boston, MA: Boston College, International Study Center. Retrieve from http://timss.bc.edu/pirls2001i/pirls2001_pubs_ir.html 
Mullis, I., Martin, M., Kennedy, A., \& Foy, P. (2007). PIRLS 2006 international report: IEA's progress in international reading literacy study in primary schools in 40 countries. Chestnut Hill, MA: TIMSS and PIRLS International Study Center, Lynch School of Education, Boston College. Retrieved from http://timss.bc.edu/pirls2006/intl_rpt.html

Naglieri, J. A. (2006). Nonverbal assessment of ability: What is it? Digest of Gifted Research. Retrieved from https://tip.duke.edu/node/769

Naglieri, J. A. (1999). Essentials of CAS assessment. New York, NY: Wiley.

Naglieri, J. A., \& Ford, D. Y. (2005). Increasing minority children's participation in gifted classes using the NNAT: A response to Lohman. Gifted Child Quarterly, 49, 29-36.

Naglieri, J. A., \& Ford, D. Y. (2003). Addressing underrepresentation of gifted minority children using the Naglieri Nonverbal Ability Test (NNAT). Gifted Child Quarterly, 47, 155-160. doi:10.1177/001698620304700206

Naglieri, J. A., \& Ronning, M. E. (2000). The relationship between general ability using the Naglieri Nonverbal ability test (NNAT) and Stanford Achievement Test (SAT) Reading Achievement. Journal of Psychoeducational Assessment, 18, 230-239. doi:10.1177/073428290001800303

Neisser, U., Boodoo, G., Bouchard, Jr., T. J., Boykin, A.W., Brody, N., Ceci, . . Urbina, S. (1996). Intelligence: Knowns and unknowns. American Psychologist, 51, 77101.

Newman, I., Fraas, J., \& Laux. (2000). A three-step adjustment procedure for type I error rates. Journal of Research in Education, 10, 84- 90.

Newman, I., McNeil, K., \& Frass, J. (2004). Two methods of estimating a study's replicability. Mid-Western Educational Researcher, 17, 36-40.

Newman, I., Newman, C., Brown, R. \& McNeely, S. (2006). Conceptual statistics for beginners $\left(3^{\text {rd }}\right.$ ed.). Lanham, MD: University Press of America.

Nonverbal Aptitude. (1993). Annotated bibliography of tests (p. 132). Princeton, NJ: Educational Testing.

Nunnally, J. (1967). Psychometric theory (p. 334). New York, NY: McGraw-Hill. 
Oblinger, D. (2001). The world is getting smaller, but are we seeing farther? Educause Review, 36, 62-63.

Open Doors 2015. (2015) Institute of International Education. Bureau of Educational and Cultural Affairs. Retrieved from http://www.iie.org/en/Who-We-Are/News-andEvents/Press-Center/Press-Releases/2015/2015-11-16-Open-Doors-Data

Pajares, F. (2002). Overview of social cognitive theory and of self-efficacy. Retrieved from http://www.emory.edu/EDUCATION/mfp/eff.html

Projections of Education Statistics to 2022. National Center for Educations Statistics. U.S. Department of Education. Retrieved from https://nces.ed.gov/pubs2014/2014051.pdf

Raven, J. C. (1936). Mental tests used in genetic studies: The performances of related individual in tests mainly educative and mainly reproductive. M. Sc. Thesis, University of London.

Raven's Progressive Matrices (APM): Norms composition tables. (2012). Upper Saddle River, NJ: NCS Pearson.

Raven's Progressive Matrices (SPM): Norms composition tables. (2012). Upper Saddle River, NJ: NCS Pearson.

Reio, T. (2016). Nonexperimental research: Strengths, weaknesses, and issues of precision. European Journal of Training and Development, 40.

Rushton, J. P., \& Jensen, A. R. (2003). African-White IQ differences from Zimbabwe on the Wechsler Intelligence Scale for Children - Revised are mainly on the $g$ factor. Personality and Individual Differences, 34, 177-183. Retrieved from http://www.psychology.uwo.ca/faculty/rushtonpdfs/RushtonJensen2003PAID.pdf

Sackett, P. R., Kuncel, N. R., Arneson, J. J., Cooper, S. R., \& Waters, S. D. (2009). Socioeconomic status and the relationship between the SAT and freshman GPA: An analysis of data from 41 colleges and universities. New York, NY: The College Board. Retrieved from http://research.collegeboard.org/sites/default/files/publications/2012/9/researchrep ort-2009-1-socioeconomic-status-sat-freshman-gpa-analysis-data.pdf

Seligman, M. (2007). What you can change... and what you can't. London, England: Nicholas Brearley. 
Shaunessy, E., Karnes, F., \& Cobb, Y. (2004). Assessing potentially gifted students from lower socioeconomic status with nonverbal measures of intelligence. Perceptual \& Motor Skills, 98, 1129-1138

Sivan, E. (1986). Motivation in social constructivist theory. Education Psychologist, 21, 209-233.

Spearman, C. (1927). The abilities of man: Their nature and measurement. New York, NY: Macmillan.

SSATB: Home. (n. d.). Retrieved from http://www.admission.org

Sternberg, R. J. (2002). Intelligence is not just inside the head: The theory of successful intelligence. In J. Aronson (Ed.), Improving academic achievement (pp. 227-244). San Diego, CA: Academic Press.

Stoneberg, B. D. (2004). A study of Gender-based and ethnic-based differential item functioning (DIF) in the Spring 2003 Idaho Standards Achievement Tests Applying the Simultaneous Bias Test (SIBTEST) and the Mantel-Haenszel ChiSquare Test: Idaho Standards Achievement Tests; Reading, Language Usage, and Mathematics, Grades 4, 8, and 10. Idaho State Department of Education. Retrieved from http://files.eric.ed.gov/fulltext/ED489949.pdf

Study in the States. (n. d.). Department of Homeland Security. Retrieved from https://studyinthestates.dhs.gov/kindergarten-to-grade-12-students

Suzuki, L., \& Gurkin, T. (1993, August). Racial/ethnic ability patterns on the WISC-R and theories of intelligence. Paper presented at the $101^{\text {st }}$ Annual Convention, American Psychological Association, Toronto, Canada.

Technical information and normative data: Naglieri Nonverbal Ability Test Second Edition. (2011). Upper Saddle River, NJ: NCS Pearson.

Test-Prep Online. (nd). (Untitled illustrations of NNAT type problems). Retrieved August 13, 2016 from https://www.testprep-online.com/nnat-practicetest.aspx?affiliateID $=10337$

Twist, L., \& Sainsbury, M. (2009). Girl friendly? Investigating the gender gap in national reading tests at age 11. National Foundation for Educational Research, UK. Educational Research, 51, 283-297. doi:10.1080/00131880902892089

Understanding Assessment: How Does Linguistic Bias Affect Language Evaluations? (2013). Leaders Project. Retrieved from http://www.leadersproject.org/ 2013/03/01/how-does-linguistic-bias-affect-language-evaluations/ 
Valencia, R., \& Suzuki, L. (2001). Intelligence testing and minority students:

Foundations, performance factors, and assessment issues. Thousand Oaks, CA:

Sage.

Vera, M., Le Blanc, P. M., Taris, T. W., \& Salanova, M. (2014). Patterns of engagement: The relationship between efficacy beliefs and task engagement at the individual versus collective level. Journal of Applied Social Psychology, 44, 133-144. doi: 10.1111/jasp. 12219

Verney, S. P., Granholm, E. Marshall, S. P., Malcarne, V. L., \& Saccuzzo, D. P. (2005). Culture-fair cognitive ability assessment: Information processing and psychophysiological approaches. Assessment, 12, 303-319. doi:10.1177/1073191105276674

Villarreal, C. (2005). An analysis of the reliability and validity of the Naglieri Nonverbal Ability Test (NNAT) with English Language Learner (ELL) Mexican American children (Unpublished doctoral dissertation). Texas A\&M University, College Station, TX.

Watkins, M., Lei, P., \& Canivez, G. L. (2007). Psychometric intelligence and achievement: a cross-lagged panel analysis. Intelligences, 35, 59-68.

Wechsler, D. (2003). WISC-IV; Wechsler Intelligence Scale for Children $\left(4^{\text {th }}\right.$ ed.). San Antonio, TX: Psychological Corporation.

Whitworth, R., \& Gibbons, R. (1986). Cross-racial comparison of the WAIS and WAISR. Educational and Psychological Measurement, 46, 1041-1049.

WIDA: Home. (n. d.) Retrieved from https://www.wida.us/index.aspx

Zimmerman, B. J. (2000). Self-efficacy: An essential motive to learn. Contemporary Educational Psychology, 25, 82-91. doi:10.1006/ceps.1999.1016

Zwick, R., \& Himelfarb, I. (2014) Measures for a college and career indicator: Research brief on SAT \& ACT. California Department of Education, Educational Policy Improvement Center. Retrieved from http://www.cde.ca.gov/ta/ac/pa/documents/apr14it 


\begin{abstract}
Appendix A
Public and private Venezuelan schools that give "Mathematics" each year with no class identification. Additionally, they give Biology, Physics, and Chemistry over the course of three years, each being taught a limited number of times per week.
\end{abstract}




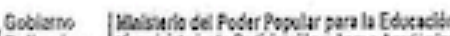

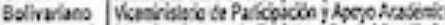

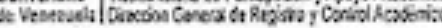

\section{CERTIFICACIÓN DE CALIFICACIONES \\ Código del Formato: RR-DEA-03-03}

L. Plan de Estudios EOUCHCION MEDIA GENERAL

Mención: .

Lugar y Fecha de Expadiclón:

Códige: 22011

It. Datos del Plantel o Zona Educativa que emite ia Certificaclón:

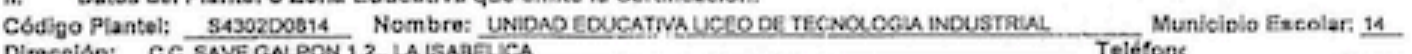
Diraceion: c.C. SAVE CNLPON 1,2, LA ISABEUCA Telefons

Municlolo: VALENCU

Entidad Federal: CarABOBO Zona Educativa:

III. Datos de Identifleación de! Estudiante:

Cédula de ldentidad: V-20neszs

Apellidos:
Lugar de Navmususu.

Fecha de Nacimlento:

$28 \cos$ Abril de $190 \mathrm{~s}$

IV. Plantales donde cursó estudios:

Ne Nombere del Plentel

\begin{tabular}{|c|c|c|c|c|c|}
\hline $\mathrm{s}^{*}$ & Nombre del Plental & Localidad & E.F. & 3 & \\
\hline 1 & ufuctes ntmascanastex & VALENC:U & ca & 4 & $\cdots$ \\
\hline
\end{tabular}

2 . . . . . . . .

V. Ponsum te estudios:

\begin{tabular}{|c|c|c|c|c|c|c|}
\hline Primere & \multicolumn{2}{|c|}{ Callificación } & \multirow{2}{*}{$T-E$} & \multicolumn{2}{|c|}{ Eashs } & \multirow{2}{*}{$\begin{array}{c}\text { Plantel } \\
N^{\circ}\end{array}$} \\
\hline Asigeatumas & $\operatorname{En} x^{*}$ & Endetras & & Nat & Afo & \\
\hline CASTELLNO Y UTERATURA & 19 & orecsusve & $F$ & 57 & 2013 & 1 \\
\hline INGLES & 20 & velore & $F$ & 57 & 2013 & 1 \\
\hline MATEMATICA & 20 & VEWTE & $F$ & \multicolumn{2}{|c|}{$57 \div 2012$} & 1 \\
\hline FSTUDIOS DE LANATURALEZA & 20 & VEINTE & $F$ & \multicolumn{2}{|c|}{ G. 2013} & 1 \\
\hline HISTCAUA DE VENEZUELA & 20 & VENTE & $\mathrm{F}$ & \multirow{2}{*}{\multicolumn{2}{|c|}{$\mid$\begin{tabular}{l|l|}
57 \\
57 & 2013 \\
\end{tabular}}} & 1 \\
\hline FDUCACION FAULLARY CLUDADANA & 20 & VENTE & $F$ & & & 1 \\
\hline GEOGRAFU GENLRAL & 20 & VENTE & $F$ & \multicolumn{2}{|c|}{$167 \cdot 2013}$. & 1 \\
\hline EDUCBCIONARTISTICA & 18 & GIECINUEVE & F & \multicolumn{2}{|c|}{$-57 \div 2013$} & 1 \\
\hline EDSXCACION FISICA Y CEPORTE & 20 & VEINTEL. & $F$ & \multicolumn{2}{|c|}{$5 ? 2013:$} & 1 \\
\hline EDUCACIQN PARA EL TRABAND & 18 & DIECIOCHO & f & \multicolumn{2}{|c|}{$C Z: 2013$} & 1 \\
\hline$\cdots \cdots$ & $*$ & $\ldots \ldots$ & 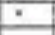 & {$[*$} & \multirow[t]{2}{*}{$+\ldots$} & $\cdot$ \\
\hline & & & & & & \\
\hline & & & & & & \\
\hline Sesunde & \multicolumn{2}{|c|}{ Callificación. } & & \multicolumn{2}{|c|}{ Eecha } & \multirow{2}{*}{\begin{tabular}{|c|}
$\begin{array}{c}\text { Plantel } \\
\mathrm{N}^{*}\end{array}$ \\
\end{tabular}} \\
\hline Aslgeatazas & En $N^{*}$ & Folatras & T.E & \multicolumn{2}{|c|}{ Mas Afa } & \\
\hline CASTELLANOYUTERATUAA & 19 & CIECINUFVE & F & \multirow{2}{*}{\multicolumn{2}{|c|}{\begin{tabular}{l|l}
57 & 2014 \\
67 & 2014
\end{tabular}}} & 1 \\
\hline NOLES & 22 & YEINIE & 8 & & & 1 \\
\hline MATEMATISA & 20 & VEINTE & F & \multicolumn{2}{|c|}{\begin{tabular}{|l|l|} 
CI & 2014 \\
\end{tabular}} & 1 \\
\hline EOUCACION PABA LA SALUD & 20 & VENTE & F & \multicolumn{2}{|c|}{ s7 2011} & 1 \\
\hline CIENCIAS BICLOGECAS & 20 & VENTE & $F$ & \multicolumn{2}{|c|}{\begin{tabular}{|l|l|} 
67 & 2014 \\
\end{tabular}} & 1 \\
\hline HISTOAS DE VENEZUELA & 20 & VENTE & $F$ & \multicolumn{2}{|c|}{$67 \quad 2014$} & 1 \\
\hline HISTORIA VNIVERSAL & 19 & DIECNUEVE & $F$ & \multicolumn{2}{|c|}{ C7 2014} & 1 \\
\hline GCUCACIONARTISTICA & 20 & VENTE. & $F$ & S2 & 2014 & 1 \\
\hline EDUCACIOY FISICA Y OEPORTE & 20 & VEWTE & $\varepsilon$ & 62 & 2014 & 1. \\
\hline EDSOCACION PABA FL TRABANO & 20 & VEVIE & $f$ & 62 & 2014 & 1 \\
\hline$\cdots \cdots$ & $\cdots$ & $\ldots$ & $\cdot$ & $\because$ & $\cdots \cdot$ & $\cdot$ \\
\hline & & & & & & \\
\hline & & & & & & \\
\hline Terseto & & allifisación. & & $F_{2}$ & eha & Plsentel \\
\hline Aslgeaturas & $\operatorname{En} \mathrm{N}^{*}$ & Endotras & $T \cdot E$ & Mis & $A=0$ & $\mathrm{~N}^{0}$ \\
\hline CASTELLANO YUTERATURA & 20 & VEINTE & F & $S$ & 2015 & 1 \\
\hline INGLES & 20 & VENTE & $F$ & ! $\$ 2$ & 2015 & 1 \\
\hline MATEMANCA & 20 & VENTE & $F$ & $\therefore 52$ & 2015 & 1 \\
\hline CIEMCIAS BIOLOSICAS & 20 & VENTE & $F$ & 32 & 2015 & 1. \\
\hline FISICA & 19 & DUECNUENE & $F$ & $=62$ & 2015. & 1 \\
\hline QUNISA & 20 & YEIVTE & $E$ & 152 & 3015 & 1 \\
\hline HSTORIA DE VENEZUELA & 19 & DECONEVE & $F$ & 52 & 2015 & 1 \\
\hline GEOCRAFIA DE VENEZULLA & 19 & DEC MUEVE & $F$ & 52 & 2015 & 1 \\
\hline EDUCACIONFISICA YOLPORTE & 18 & DECINUEVE & F & $\because \Omega$ & $2015=$ & $1^{4}$ \\
\hline EDUCACION PARA EL TRABANO & 20 & YEINTE, & $F$ & 57 & 2015 & 1. \\
\hline$\ldots \ldots$ & $*$ & $\ldots \ldots$ & 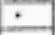 & $\because$ & $\cdots$ & 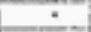 \\
\hline$\frac{1}{1}$ & 1 & & & & & \\
\hline & & & & & & \\
\hline & & & & & & \\
\hline
\end{tabular}

\begin{tabular}{|c|c|}
\hline Lecallass & E.F. \\
\hline$\ldots \ldots$ & 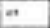 \\
\hline$\ldots \ldots$ & - \\
\hline$\ldots \ldots$ & $=$ \\
\hline
\end{tabular}

VI, DIRECTOR[A] DEL PLANTEL. Apellidos $\vee$ Nombres:

Número de $C_{1}$

V-9022798

Flrma:

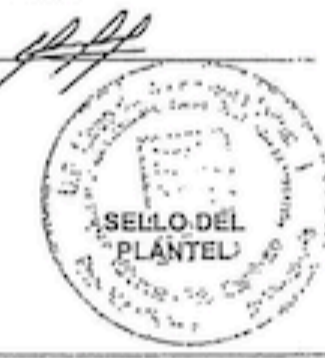

Para elacios de auvelicas onvel ealack.

VII. DIRECTOR(A) OE LA ZONA EOUCATIVA

Apolildos y Nombres:

Número de C.l.:

Firma:

SELLO DE LA ZONA EDUCATIVA

Para Elecios de ay rabist a nive

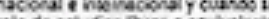

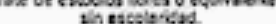

TIMBRE FISCML: Este Osoumenta cane viserz ai no at cobcuh en ty para

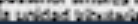


Ministero deliristruxione, deVl'Universitd e dello Ricerco

ISTIUTO COMPRENSIVO "R. F. EVOLA"

Anno Scolastico 2012/13

St informa la S.V. che, suo/a figlio/a,

della classe $\underline{2^{2} D}$

nel 1* Quadrimestre, ha ottenuto nelle sotto indicate discipline le seguenti valutazionl:

\begin{tabular}{|c|c|c|}
\hline DISCIPUNE & VOTO & ASSENZE \\
\hline Italiano & Sह11 & 12 \\
\hline Inglese & of 0 & to \\
\hline Francese & oiro & $152 x 6$ \\
\hline Storla & $\sqrt{10}$ & 6 \\
\hline Gecgrafia & Sine & 4 \\
\hline Matematica & OTTO & 10 \\
\hline Scienze & orlo & 4 \\
\hline Tecnologia & Sive & 5 \\
\hline Musica & oito & $z$ \\
\hline Arte e lmmagine & ófo & 8 \\
\hline Sclenze Motorie & ofro & 3 \\
\hline Comportamento & Move & \\
\hline \multicolumn{3}{|l|}{ T.P. - Laboratorio 1} \\
\hline \multicolumn{3}{|l|}{ T.P. - Laboratorio 2} \\
\hline Religione & Bung & 3 \\
\hline
\end{tabular}

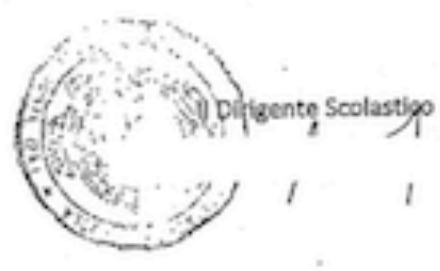

Balestrate, $05 / 02 / 2013$ 
Public South Korean school that gives "Mathematics" each year with no class identification 


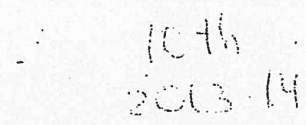

\section{Academic Record}

[The First Year]

\begin{tabular}{|c|c|c|c|c|c|c|}
\hline \multirow[b]{2}{*}{ Curriculum } & \multirow[b]{2}{*}{ Subject } & \multicolumn{2}{|c|}{$1^{\text {si }}$ Semester } & \multicolumn{2}{|c|}{ 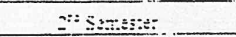 } & \multirow[t]{2}{*}{$\therefore::$} \\
\hline & & Grade & $\begin{array}{c}\text { Rank (Same } \\
\text { rank) } \\
\text { Total number of } \\
\text { students }\end{array}$ & Grade & $\begin{array}{l}\text { number of } \\
\text { students }\end{array}$ & \\
\hline Korean & Korean & B & $238(2) / 476$ & $\mathrm{~A}$ & $96(5) / 494$ & \\
\hline Ethics & Ethics & A & $106(2) / 476$ & $A$ & $39(2) / 494$ & \\
\hline Social Studies & Social Studies & D & $313(6) / 476$ & C & $253(9) / 494$ & \\
\hline Math & Math & $D$ & $249(3) / 476$ & B & $152(8) / 494$ & \\
\hline Science & Science & $\mathrm{C}$ & $320(3): 476$ & C & $267(3) / 494$ & \\
\hline Technology \& Home Economics & $\begin{array}{c}\text { Technology \& Home } \\
\text { Economics }\end{array}$ & B & $231(3) / 476$ & $\mathrm{~A}$ & $95(4) / 494$ & \\
\hline Foreign Language (English) & English & A & $111(2): 476$ & A & $111(2) / 494$ & \\
\hline Discretion & Japanese & D & $307() / 476$. & $\mathrm{~A}$ & $175(3) \div 494$ & \\
\hline Discretion & Chinese Characters & $\mathrm{C}$ & $230(2) / 476$ & $B$ & $144(3) / 494$ & \\
\hline
\end{tabular}

Subject Detailed ability and special ability articles

Math: He has shown a high answering rate in question which asks about basic concept and using skill. along with $\quad: 2: \ldots \ldots$

an outstanding skill of interference.

Issued the After School Korean History Certificate Class $\left(3^{\text {rd }}, 4^{\text {th }}-5 \%\right.$ hours), Reading Essay Class $14 \div-:=$ hours:

Received the $4^{\text {th }}$ Level of Chinese Certificate in 2010

$<$ P.E, Music, Arl>

\begin{tabular}{|c|c|c|c|c|}
\hline \multirow[t]{2}{*}{ Curriculum } & \multirow[t]{2}{*}{ Subject } & $1^{\text {s }}$ Semester & $2^{\text {nd }}$ Scmester & \multirow[t]{2}{*}{$x: z$} \\
\hline & & Level & Level & \\
\hline P.E & P.F. & Avcrage & Exccllence & \\
\hline$A \pi$ & Ant & Execllence & Average & \\
\hline Music & Music & Average & Excellence & \\
\hline Subject & & & & \\
\hline
\end{tabular}

[The Second Year]

\begin{tabular}{|c|c|c|c|c|c|c|}
\hline \multirow[b]{2}{*}{ Curriculum } & \multirow[b]{2}{*}{ Subject } & \multicolumn{2}{|c|}{$1^{\text {s" Semcster }}$} & \multicolumn{2}{|c|}{$2^{\text {id }}$ Semester } & \multirow[t]{2}{*}{ Note } \\
\hline & & Gradc & $\begin{array}{c}\text { Rank (Same } \\
\text { rank)! } \\
\text { Total number of } \\
\text { students }\end{array}$ & Grade & $\begin{array}{c}\text { Rank (Same } \\
\text { rank)/Jotal } \\
\text { number of } \\
\text { students }\end{array}$ & \\
\hline Korean. & Korean & A & $72(2) / 480$ & B & 103 (4)/465 & \\
\hline Ethics & Ethics & $B$ & $197(2) / 480$ & C & $203(3) / 465$ & \\
\hline Social Studies & Social Studies & B & $180() / 480$. & $\mathrm{~F}$ & $322() ; 465$. & \\
\hline Math & Math & C & $224() / 479$. & $D$ & $256(2) ; 465$ & \\
\hline Science & Science & B & $272() / 480$. & C & $239(2): 465$ & \\
\hline Technology \& Home Fconomics & $\begin{array}{c}\text { Technology \& Home } \\
\text { Economics }\end{array}$ & C & $225(5) / 480$ & B & $156(2) ; 465$ & \\
\hline Foreign Language (English) & English & B & $164(2) / 480$ & B & $184() 465$. & \\
\hline Discretion & Japanesc: & $A$ & $138(4) / 480$ & A & $1(54) / 465$ & \\
\hline
\end{tabular}




\section{Appendix B}

Public Venezuelan school that gives grades that go up to 20 


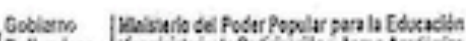

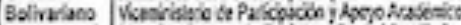

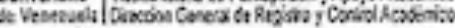

\section{CERTIFICACIÓN DE CALIFICACIONES Código del Formato: RR-DEA-03-03}

L. Plan de Estudios EQUCICION MEDIA GENERAL

Lugar y Facha de Expadiclon:
Código: 22011

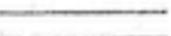

It. Datos del Plante! o Zona Educativa que emite la Certificación:

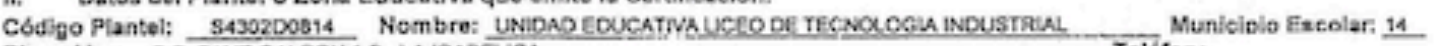
Diraceión: c.C. SAVE GN.PON 1, 2, LA ISABEUCA

Municiplo: VALFNCUA Entidad Federal: CaraBOBO Zona Educativa:

III. Datos de Identifleación del Estudiante:

Cédula de Identidad: V.20moszo

Apellidos:

Lugar de Navmananu.

Fecha de Nacimiento:

28 en Abril de 1998.

IV. Plantales donde cursó estudios:

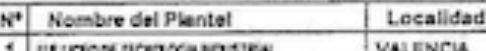

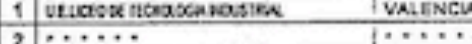

$\begin{array}{lll}2 & \cdots \cdots \\ \text { V. Pensum de estudios: }\end{array}$

\begin{tabular}{|c|c|c|c|c|c|c|}
\hline Primere & \multicolumn{2}{|c|}{ Callificación } & \multirow{2}{*}{$T-E$} & \multicolumn{2}{|c|}{ Eachs } & \multirow{2}{*}{$\begin{array}{c}\text { Plantel } \\
\mathrm{N}^{\circ}\end{array}$} \\
\hline Asigeaturas & En: $x^{*}$ & Enletras & & Mas & Afn & \\
\hline CASTEUANOY YTERATURA & 19 & orfcrusvi & $F$ & 57 & 2013 & 1 \\
\hline INGLES & 20 & velve & $\mathbf{F}$ & $\$ 7$ & 2013 & 1 \\
\hline MATEMATICA & 20 & VEWTE & F & \multicolumn{2}{|c|}{5712012} & 1 \\
\hline FSTUDIOS DE UANATURALEZA & 20 & VEINTE & $F$ & \multicolumn{2}{|c|}{ C7. 2013} & 1 \\
\hline HISTCAUA DE VENEZVELA & 20 & VEINTE & $\mathrm{F}$ & 57 & 2013 & 1 \\
\hline EDUCACION FAULLARY CUDADANA & 20 & VENTE & $\mathrm{F}$ & 57 & 2013 & 1 \\
\hline SEOCGRAFI GENIRAL & 29 & VENTE & $F$ & \multirow{2}{*}{\multicolumn{2}{|c|}{$\begin{array}{ll}c 7: 2013 \\
67: 2013\end{array}$}} & 1 \\
\hline EDUCACIONARTISICA & 18 & GIECNUEVE & $f$ & & & 1 \\
\hline EDIXACION FISICA Y CEPORTE & 20 & VIINTE. & F & \multicolumn{2}{|c|}{$67=2013$} & 1 \\
\hline EDUCACION PARA EL TRABA 10 & 15 & DIFCIOCHO & F & CZ & 2013 & 1 \\
\hline$\cdots \cdots$ & $\ddot{*}$ & $\cdots \cdots$ & 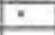 & 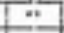 & w..... & $\cdot$ \\
\hline & & & & - & & \\
\hline & & & & & & \\
\hline & & & & & & \\
\hline Seginde & \multicolumn{2}{|c|}{ Calificación. } & \multirow[b]{2}{*}{$\mathrm{T} \cdot \mathrm{E}$} & \multicolumn{2}{|c|}{ Eecha } & \multirow{2}{*}{$\begin{array}{c}\text { Plantel } \\
\mathrm{N}^{*}\end{array}$} \\
\hline $\begin{array}{l}\text { Ane: Seginde } \\
\text { Aslgeahuras }\end{array}$ & En $N^{*}$ & Eolotras & & Mus & 4.6 & \\
\hline CASTELLANOYUTERATURA & 18 & CIECINUFVE & $F$ & 57 & 2014 & 1 \\
\hline NOLES & 22 & YENINTE & $F$ & 57 & 2014 & 1 \\
\hline MATEMATISA & 20 & VEINTE & E & $C L$ & 2014 & 1 \\
\hline EOUCACION PARA LA SALUD & 20 & VENTE & 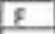 & $5 ?$ & 2011 & 1 \\
\hline CIENCIAS BIOLOGICAS & 22 & VENTE & $f$ & 57 & 2014 & 1 \\
\hline HISTOPUA DE VENEZUELA & -20 & VEINTE & F & 57 & 2014. & 1 \\
\hline HISTORIA UNIVERSAG & 19 & DIECNUEVE & $F$ & 57 & 2014 & 1 \\
\hline GCUCACIONARTISTICA & 20 & VEVIE & $F$ & 52 & 2014 & 1 \\
\hline SOUCACIOY FISICA Y OEPORTE & 20 & VENTE & $E$ & 62 & 2014 & 1. \\
\hline EDUCACION PARA FL TRABANO & 20 & VEVIE & $F$ & 52 & 2014 & 1 \\
\hline$\cdots \cdots$ & $\cdots$ & $\cdots$ & - & $\cdots$ & $\cdots$ & $\cdot$ \\
\hline & & & & & & \\
\hline & & & & & & \\
\hline & \multicolumn{2}{|c|}{ Callifteación } & & Facha & eblo & \multirow{2}{*}{$\begin{array}{c}\text { Plantel } \\
\mathrm{N}^{\circ}\end{array}$} \\
\hline $\begin{array}{cc}\text { ARo: } & \text { Tersste } \\
\text { Aslgeatiuras }\end{array}$ & $\operatorname{En} \mathrm{N}^{*}$ & $\begin{array}{l}\text { Aulicacion } \\
\text { Endotras }\end{array}$ & $T \cdot E$ & Mis: & $A B$ & \\
\hline CASTELLANO YUITERATURA & 20 & VEINTE & $F$ & $\Omega$ & 2015 & 1 \\
\hline INGLES & 20 & VENTE & F & $s 2$ & 2015 & 1 \\
\hline MATEMANCA & 20 & VEVTE & $F$ & $=52$ & 2015 & 1 \\
\hline CIEMCIAS BIQLOGICAS & 20 & VENTE & $f$ & 52 & 2015 & 1. \\
\hline EISICA & 12 & DIECWUEVE & $F$ & 352 & 2013 & 1 \\
\hline QUNICA & 20 & XEVTE & $F$ & 152 & 2015 & 1 \\
\hline HSTORUA DE VENEZUELA & 19 & DECDNENE & $F$ & 562 & 2015 & 1 \\
\hline GEOGRAEL DE VENEZUELA & 19 & DECNUEVE & $F$ & 52 & 2018 & 1 \\
\hline EDUCACIONFISICA Y OEPORTE & 19 & DEGINUEYE & $E$ & $\therefore z$ & 2015 & 1 \\
\hline EDUCACION PARA EL TRABANO & 120 & VEINTE & $F$ & 57 & 2215 & 1. \\
\hline$\ldots \ldots$ & $*$ & $\ldots \ldots$ & $\cdot$ & $\because$ & $\cdots$ & 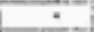 \\
\hline & 1 & & & & & \\
\hline & & & & & & \\
\hline & & & & & & \\
\hline
\end{tabular}
Nombres: Enuoao receral o Pals:

\begin{tabular}{l|l|l|l|}
$\mathrm{N}^{-}$ & Nombro del Plantel & Lecalldad
\end{tabular}

\begin{tabular}{|l|l|l|l} 
E. F. & 3 & $\ldots \ldots$ & $\ldots \ldots$ \\
\hline Ca & 4 & $\ldots$ & $\ldots$ \\
\hline
\end{tabular}

$\cdots, \cdots$

VIRECTORIAJ DEL PIANTEL Apellidos $\vee$ Nembres:

Número de C.l.:

V-9022798

Flrma:

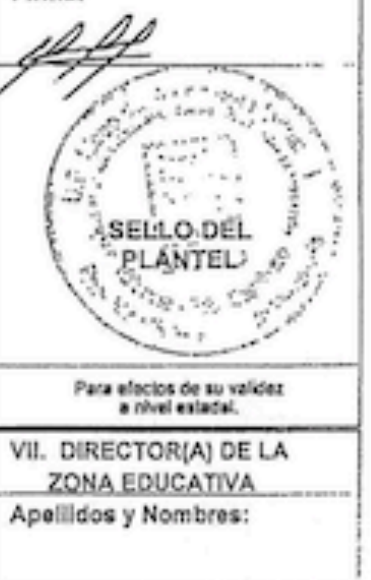

Número de C.l:

Firma:

SELLO DE LA ZONA EDUCATINA

Para elecios de ay rabita a nles

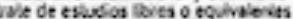
in escatericis.

TIMBRE FISCAL: Esie Oxounento or ineviscer si no at coloch th t para 
A "Canadian" school in Italy that gives numeric grades up to 100 


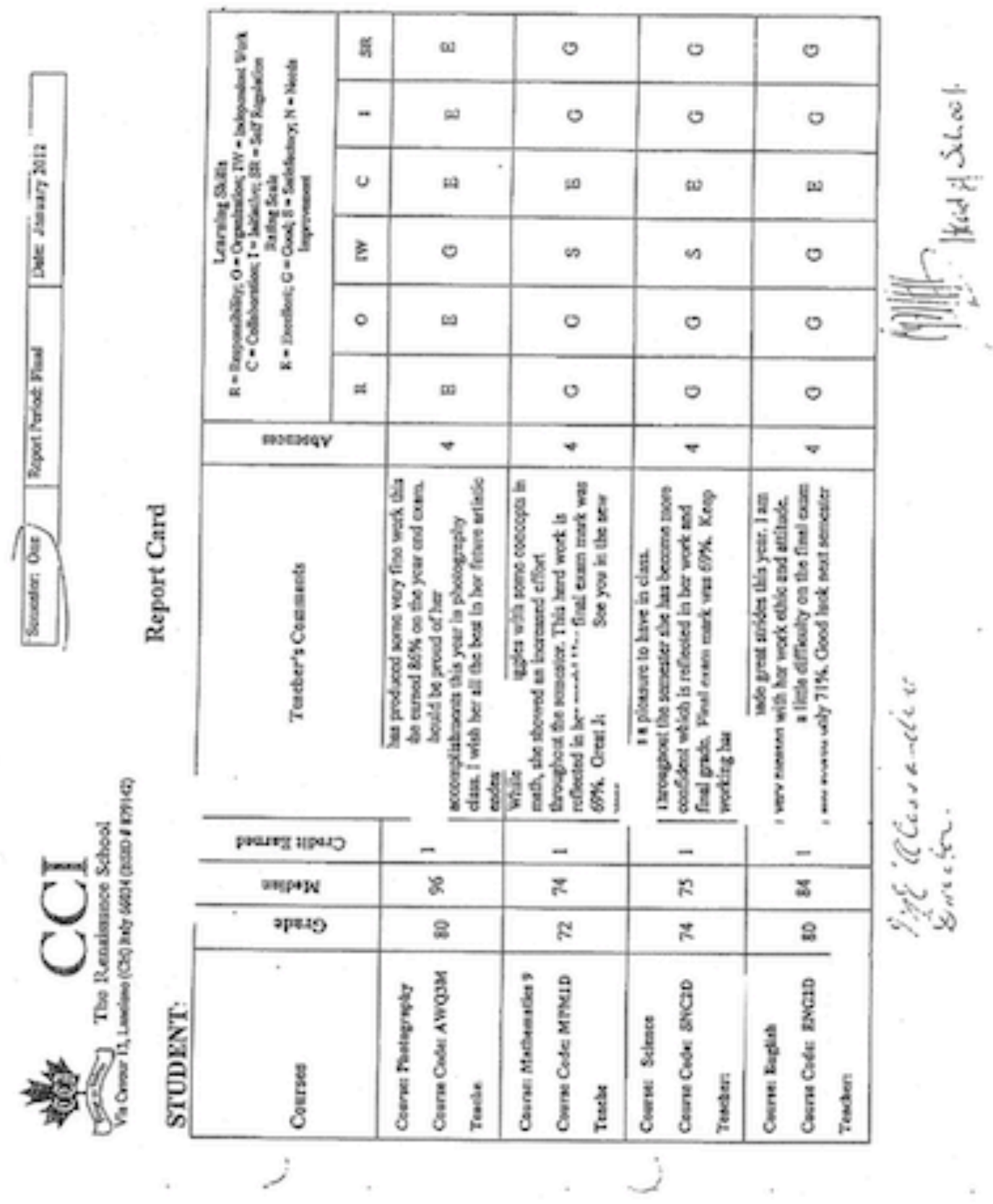


An International Baccalaureate school that gives grades of one through seven for a final grade. 


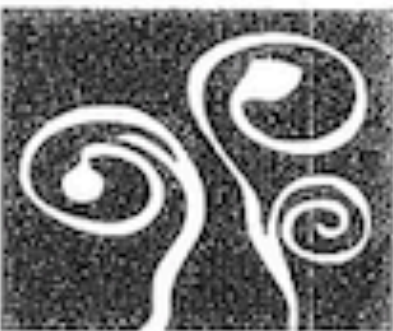

Luanda International School Eind Of Vear Report

Prepared: June 11, 2015

Sludent Neme:

Grade:

Year 12

\section{Dear Perents}

Al education at Landa internationa School is diven by the international Bascalazeate phliosophy. Wha ain to dovelop the skilts and atthuses of each member of our cornmunily so that we can meet the chatenges of timnty first century Ble whis confldonso, odaptabilly and understanding.

Whilst we highly value the scademio programme at the sohod, this is ond ane pert of the rich educasiond experience that is provided. Learning is enhanced by a wide renge of experiantiel activiles including: CAS, the Ouldecr Efeucalion Programme and the Ather School Activeles Programme.

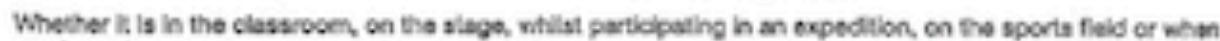
serving others, we want our students to experience the exclement of cliscovering thay are capabie of achiewing far more than they over febt was possbie. We heve high expectetions of our students and they, in turn, have high expectations of themselvas.

Wour child has now rasched a key momant in hiaher edvcational joumey and wil soon be atarting their find year of secondary oducation; Wo wish tham at the beat for sis and look fonwod to seeing all students and farrilin again in Aupust.

Kond Rlogarda

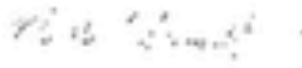

Secondary Princlpat

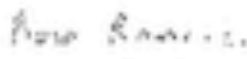

Diploms Coordinator 


\section{Class Reports}

Language A: Spanish A

Year 11 -

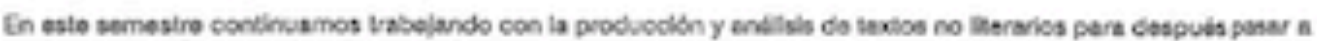

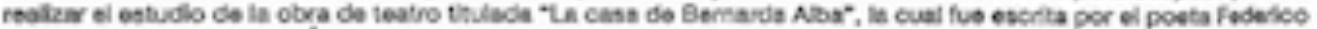
Garcla Lorca. Ento nos ha permilido elaborar desde posala havia comentarlos de textos, pasando por vihatas expleablvas de necants y eclogos a la cbra de teatro.

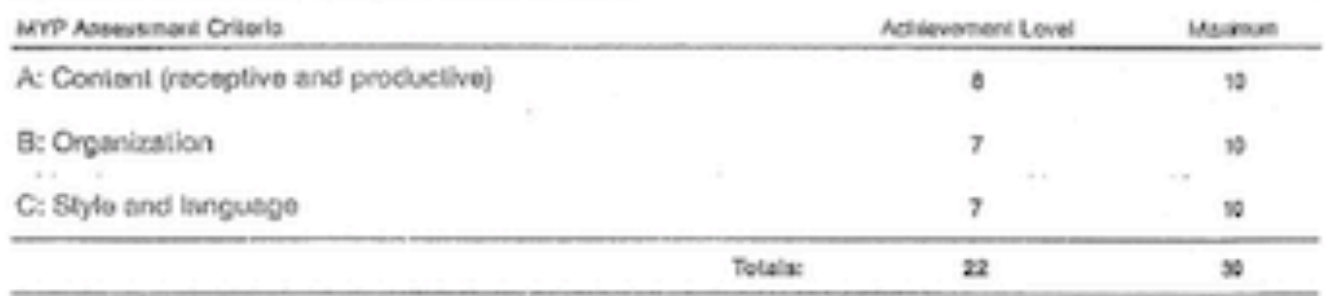

Final Groda

s

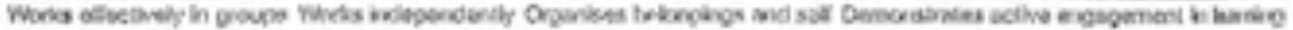
U U U

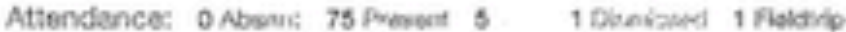

Comments

Durants este semestre

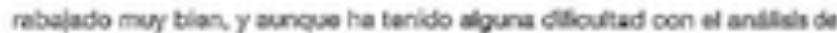

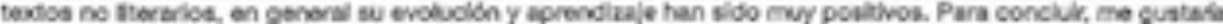

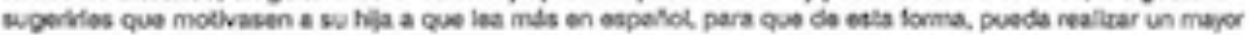
prograso en el cococimilente do esta langua.

\begin{tabular}{|c|c|c|c|c|c|c|c|}
\hline Crads & 1 & $z$ & 3 & 4 & 5 & 6 & 3 \\
\hline Beundarien & $0-4$ & $5-0$ & $10-14$ & $15-10$ & $20-23$ & $24-27$ & $23-x$ \\
\hline
\end{tabular}




\section{Appendix C}

Permissions to Use Images from Testprep-online.com 
John Althoff

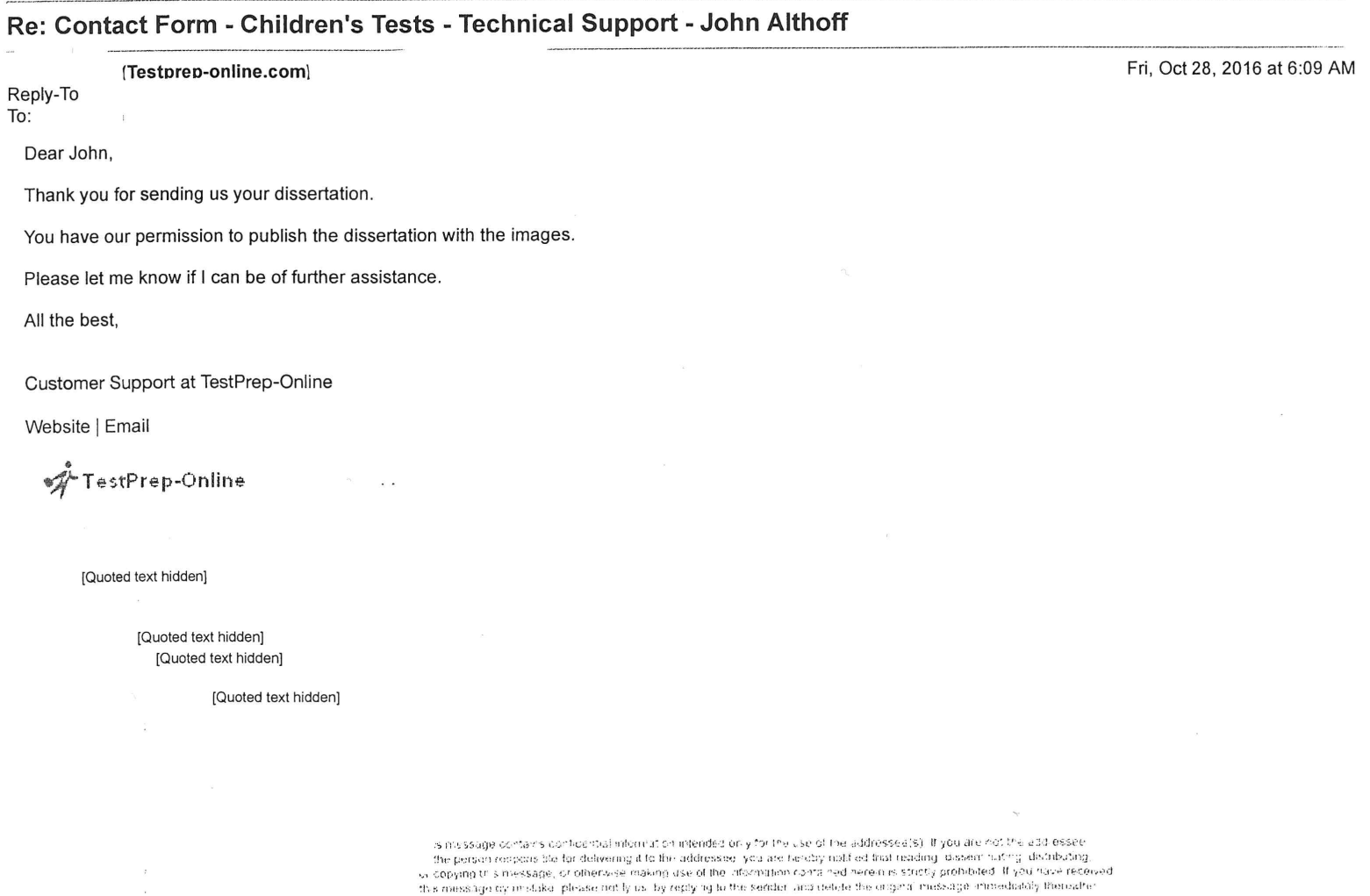


VITA

JOHN ALTHOFF

Born, Grand Rapids, Michigan

1998-2005

United States Military

2005

B.S. Social Studies Education and

B.S. Business Education

Ferris State University

Big Rapids, Michigan

2006-2013

Teacher

Divine Savior Academy

Doral, FL 33178

2008

M.S. Curriculum and Instruction

Ferris State University

Big Rapids, Michigan

2011-2016

Doctoral Candidate

Florida International University

Miami, Florida

2013-2016

Academic Dean

Divine Savior Academy

Doral, FL 33178 